\title{
Le Guide des normes minimales pour des espaces sûrs : la conception, la mise en oeuvre, et le suivi, l'évaluation et l'apprentissage
}

Population Council

Follow this and additional works at: https://knowledgecommons.popcouncil.org/departments_sbsr-pgy How does access to this work benefit you? Let us know!

\section{Recommended Citation}

Population Council. 2021. "Le Guide des normes minimales pour des espaces sûrs : la conception, la mise en oeuvre, et le suivi, l'évaluation et l'apprentissage." New York: Population Council. 


\section{POPUlation}

COUNCIL

Ideas. Evidence. Impact.
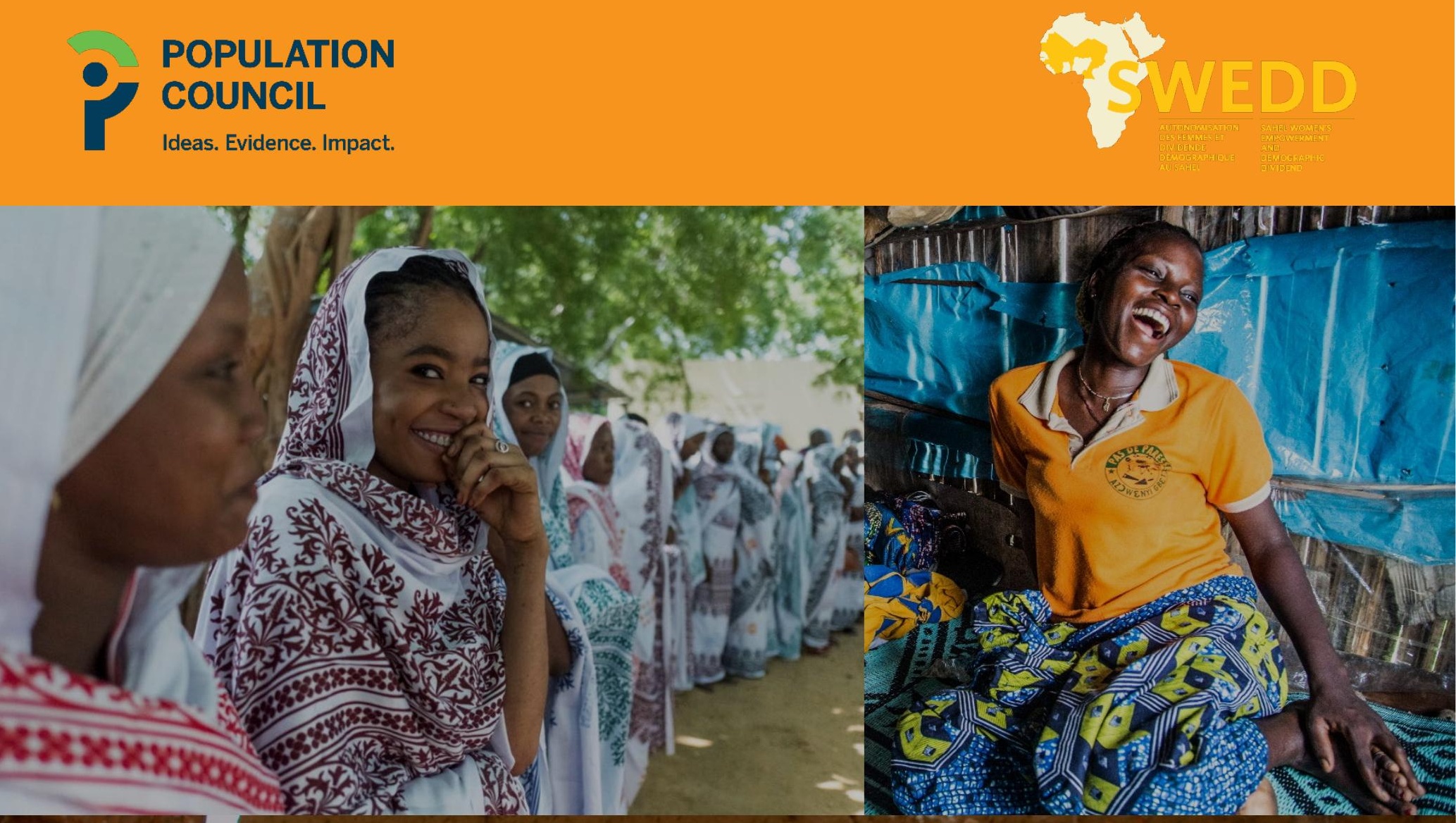

\section{Le Guide des Normes Minimales pour des} Espaces Sûrs : la conception, la mise en œuvre, et le suivi, l'évaluation et l'apprentissage

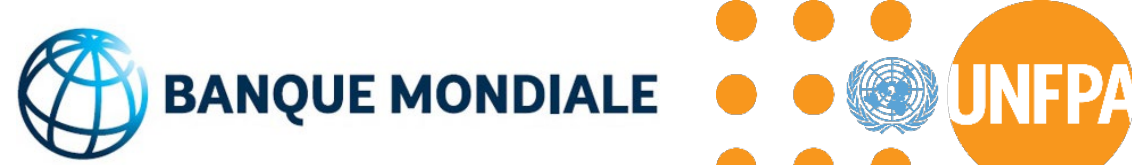


Élaboré par le Population Council

en consultation avec les équipes des pays du projet SWEDD

et des représentatifs de I'UNFPA

Mars 2021

Citation suggéré : Population Council. 2021. Le Guide des normes minimales pour des espaces sûrs : la conception, la mise en œuvre, et le suivi, l'évaluation et l'apprentissage. New York. 
Ce guide est divisé en trois sections qui décrivent les trois étapes principales de la programmation d'un espace sûr : (1) la conception, (2) la mise en œuvre, et (3) le suivi, l'évaluation et l'apprentissage. Chaque étape est composée de plusieurs sous-sections. Vous pouvez utiliser la table des matières pour identifier les sujets qui vous intéressent; si vous lisez ce guide sur un ordinateur, cliquez sur le titre pour vous rendre à la section correspondante. 


\section{TABLE DES MATIEERES}

Remerciements viii

Liste des acronymes $\quad$ ix

Glossaire des termes $\quad x$

Résumé Exécutif xii

Introduction 2

L'attention internationale sur la programmation centrée sur les filles ...................................3

Comment ce guide est-il structuré ? Comment dois-je l'utiliser pour éclairer la conception et la mise en œuvre de mon programme et du SEA ? ...................................................................

Pourquoi les AJF ont-t-elles besoin d'un "espace sûr" ? ...........................................................

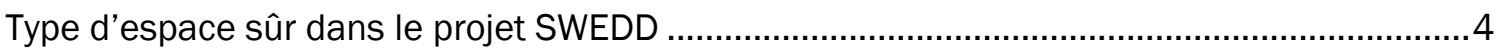

Paquet d'activités minimum dans un espace sûr.....................................................................

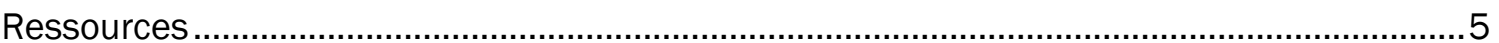

Étape 1: Conception Intentionnelle ; La conception et la planification des programmes

Une programmation conçue intentionnellement avec les AJF au centre ..................................

Des Normes Minimales pour un Ciblage et une Couverture Optimale.........................................8

Une programmation qui cible et atteint les AJF appropriées ...............................................

Comment identifier des segments des AJF les plus vulnérables, qui bénéficieront le plus de

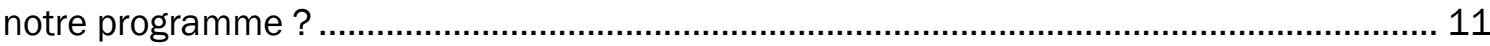

Plans intentionnels pour éviter «l'accaparement des ressources par les élites" et "l'inversion

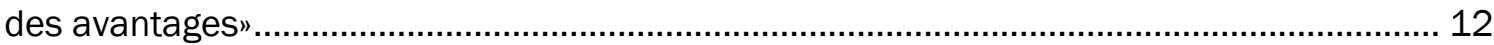

Des Normes Minimales pour l'Engagement Communautaire :............................................... 14

Comprendre l'environnement opérationnel afin d'éclairer la conception d'une programmation

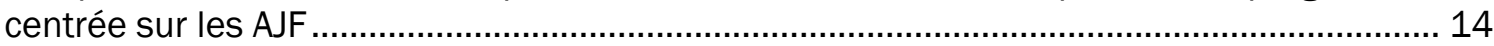

Une conception de programme ancrée dans le propre point de vue de l'AJF......................... 15

Que signifient ces spécificités de la structure d'un espace sûr pour la conception de composants d'une manière centrée sur les AJF ? ............................................................... 17

Un dialogue communautaire pour créer un environnement favorable aux espaces sûrs ..... 22

Des Normes Minimales pour une expansion de qualité des espaces sûrs ............................... 22

Combien cela va-t-il coûter ? Veiller à ce que les plans du programme d'espace sûr soient budgétisés 
Des Normes Minimales pour des Ressources Humaines Adéquates 26

Un personnel formé pour assurer une planification stratégique efficace, une mise en œuvre harmonieuse et un SEA informatif. 26

Un cadre de mentores adéquatement équipées, soutenues et habilitées............................. 27

Des Normes Minimales pour le Contenu Programmatique " Approprié ".................................... 39

Un contenu programmatique approprié pour des espaces sûrs afin de développer des "compétences" et des aptitudes à la vie quotidienne pertinentes .......................................... 39

Un ensemble essentiel d'activités pertinentes pour les espaces sûrs du SWEDD ................. 39

Des compétences de vie adaptées à l'âge pour les AJF....................................................... 42

Une planification pour un contenu adapté par segment qui tient compte de l'hétérogénéité

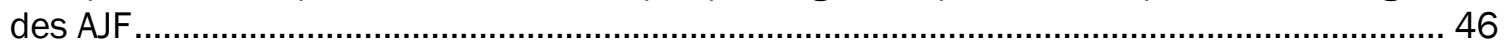

Des Normes Minimales pour la Mise en CEuvre des Sessions Engageantes et Dynamiques ... 48

Des mentores formés à l'utilisation de méthodes participatives pour encourager une participation énergique dans des groupes d'espaces sûrs.

Des mentores formées pour appliquer les principes pratiques des sessions interactives

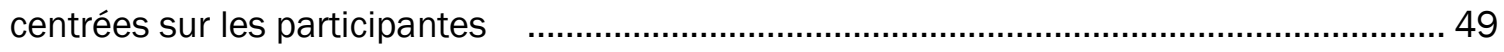

Des " astuces " pour une facilitation réussie par des mentores $\quad$......................................... 52

Des Normes Minimales pour Établir les Liens avec d'autres Ressources Communautaires qui

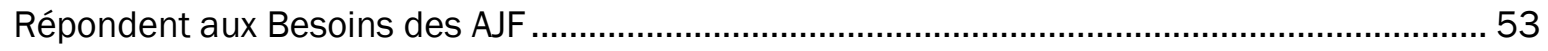

Les espaces sûrs servent de roues motrices pour relier les AJF aux services essentiels dans

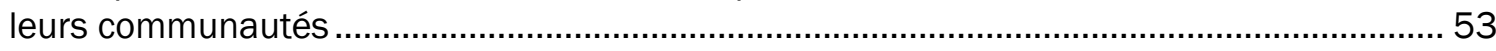

Un système de référence solide et des canaux de référence pour la violence basée sur le

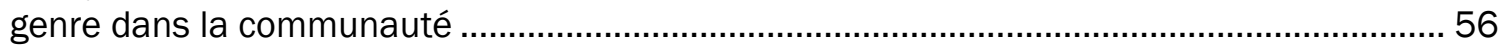

Des Normes Minimales pour Motiver et Maintenir la Fréquentation Régulière des AJF dans les

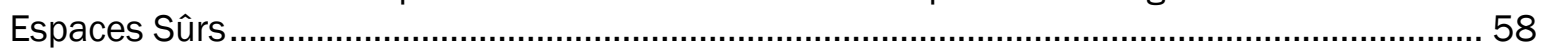

Amplifier les avantages du SWEDD en superposant les activités du SWEDD et en coordonnant

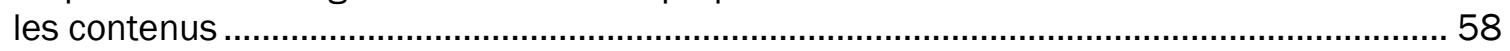

Opportunités appropriées pour l'autonomisation économique des AJF................................6 60

Stratégies efficaces pour encourager la fréquentation et maintenir la participation des 'AJF à

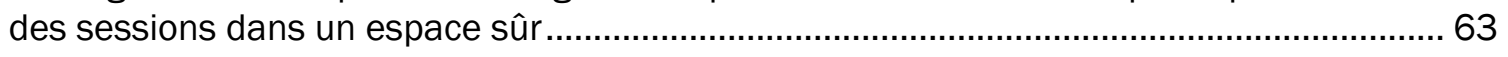

Des Normes Minimales pour Adapter la Programmation des Espaces Sûrs à LA COVID-19 et à

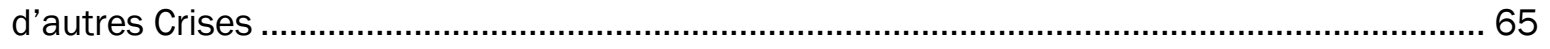

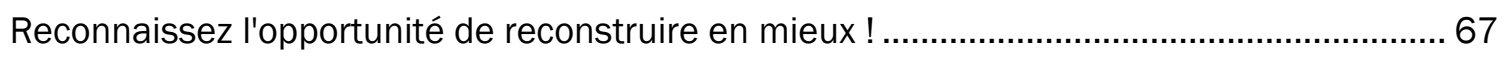


Étape 3 : Suivi, Évaluation et Apprentissage (SEA) 69 Les distinctions entre le suivi, l'évaluation et l'apprentissage et comment une "culture de l'apprentissage " peut soutenir un SEA efficace ..................................................................... 70

Des Normes Minimales pour Planifier et Exécuter des Plans de Suivi Utiles et Réalisables .... 71 Un but et un objectif clairement définis pour le suivi d'un espace sûr ................................... 71

Définir quelles données de suivi vous devez collecter pour déterminer si vous progressez vers

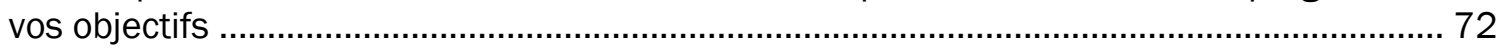

Conseils sur l'identification des indicateurs de suivi ............................................................ 76

Les bonnes pratiques : Le rôle des mentores dans le suivi ....................................................... 76

L'importance de la qualité des données statistiques sur les activités des espaces sûrs $\quad . .76$

Des Normes Minimales sur ce qu'il Mesure pour Évaluer Votre Succès ................................... 77

Un but et un objectif clairement définis pour l'évaluation des espaces sûrs ......................... 77

Déterminez les aspects de votre programme à évaluer pour mesurer son plein succès...... 78

Déterminez les indicateurs et données nécessaires pour mesurer et évaluer le succès de votre

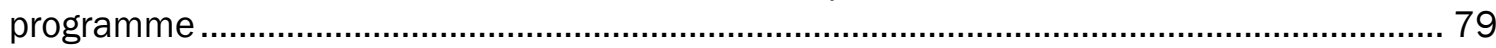

Des Normes Minimales pour l'Établissement d'un système d'Apprentissage ........................... 79

Un but et un objectif clairement définis pour l'apprentissage des espaces sûrs................... 79

Une boucle de feedback qui peut améliorer et informer la conception et la mise en œuvre 80

Des Normes Minimales pour la Collecte et l'utilisation de Données SEA de Qualité.................. 83

Utiliser des méthodes à la fois qualitatives et quantitatives pour générer des données de suivi

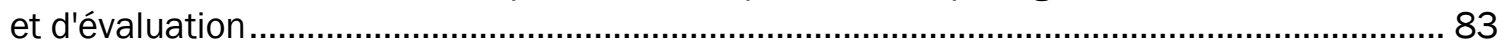

S'assurer que les données sont crédibles, valides, fiables, et utilisables ............................. 85

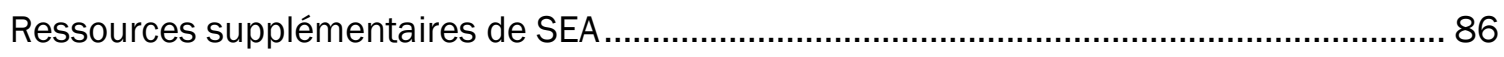

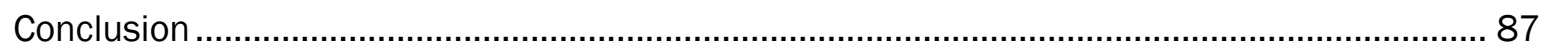

$\begin{array}{lr}\text { Appendice : Autres Lectures Recommandées } & 89 \\ \text { Contributions } & 90\end{array}$

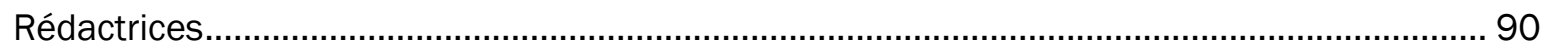

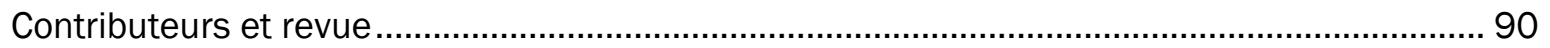

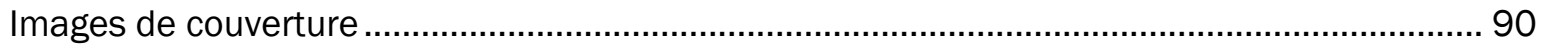


Tout au long du document, nous avons utilisé des icônes et un formatage cohérents pour indiquer certains types de contenu.
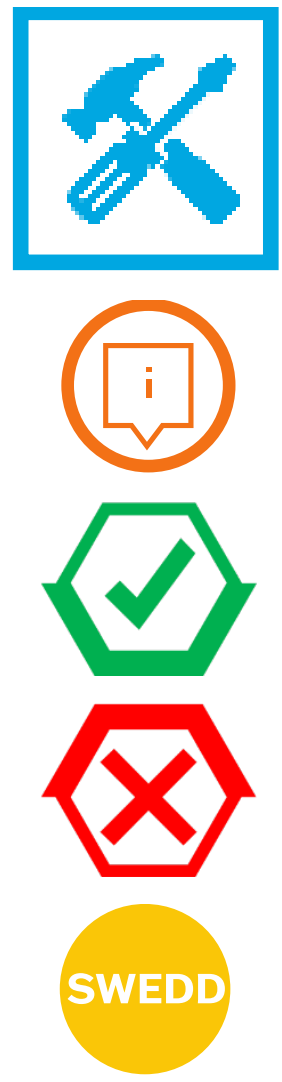

ÉTUDE

DE CAS
OUTIL : Cette icône indique les sections qui décrivent les outils qui peuvent être adaptés au SWEDD et utilisés pour acquérir des informations locales.

MESSAGE CLÉ : Cette icône indique les informations essentielles qui résument les messages clés.

À FAIRE : Cette icône indique les conseils recommandés pour améliorer la programmation

À ÉVITER : Cette icône indique les éléments à éviter et qui auront un impact négatif sur la programmation.

MATÉRIEL SWEDD : Cette icône indique que l'information provient de documents et de produits SWEDD existants.

ÉTUDE DE CAS : Les encadrés verts indiquent des études de cas présentant des expériences spécifiques et des exemples de projets antérieurs du Population Council. 
Le Guide des normes minimales pour des espaces sûrs a été élaboré dans le cadre du projet Autonomisation des femmes et dividende démographique au Sahel (Sahel Women's Empowerment and Demographic Dividend - SWEDD).

La rédaction de ce Guide a été conduite par Population Council dont le travail pour améliorer les résultats et les perspectives des adolescentes et des jeunes femmes du monde entier a rendu ce Guide possible. Les remerciements chaleureux sont adressés particulièrement au département du Population Council "Poverty, Gender and Youth " qui a été la cheville ouvrière de l'élaboration de ce document.

Ces normes ont été formulées et considérablement enrichies par les membres des équipes SWEDD du Bénin, du Burkina Faso, du Cameroun, de la Côte d'Ivoire, de la Guinée, du Mali, de la Mauritanie, du Niger, et du Tchad, et des experts I'UNFPA au niveau des bureaux pays et de la région de l'Afrique l'Ouest et Centrale. Ils ont partagé leurs connaissances et leurs expériences concrètes durant les différentes consultations. Nous les remercions sincèrement de leur contribution et de leur engagement à promouvoir la pertinence de ce Guide dans le contexte de la programmation des espaces sûrs.

Ce Guide comprend des extraits et des adaptations de nombreux documents écrits par le Population Council et d'autres, qui ont contribué à enrichir son contenu.

Enfin, nous tenons vivement à remercier la Banque Mondiale, l'OOAS, I'Union Africaine et l'UNFPA et pour leur soutien, sans lequel ce Guide des normes minimales pour des espaces sûrs n'aurait pu voir le jour. Nous adressons notre gratitude à ces entités pour les efforts qu'elles ne cessent de déployer pour autonomiser les adolescentes et les jeunes femmes. 
AGI-K Adolescent Girls Initiative-Kenya AGR Activités génératrices de revenus AJF Adolescentes et Jeunes Femmes $\mathrm{AO}$ Abriendo Oportunidades CDFM Clubs des futurs maris

CDM Clubs des maris

ONG Organisation non gouvernementale SEA Suivi, l'évaluation et l'apprentissage Sida Syndrome d'immunodéficience acquise SSR Santé sexuelle et reproductive STR Secrétariat Technique Régionale SWEDD Autonomisation des femmes et dividende démographique au Sahel UGP Unité de Gestion de Projet UNFPA Fonds des Nations Unies pour la population VBG Violences basées sur le genre $\mathrm{VIH}-$ Virus de l'immunodéficience humaine 
GLOSSAIRE DES TERMES

Bonne voie

Communauté accessible à pied

Conception intentionnelle

Couverture

Espaces sûrs

Inversion des avantages

Segmentation

Segments d'AJF

Superposition

Transformatrice au genre
(Pour les AJF) Celles qui sont dans une position d'accomplir une transition en sécurité vers l'âge adulte (en évitant le mariage et les grossesses précoces), et non celles qui sont " marginalisées ".

Des ressources et des structures qui sont accessibles aux AJF, surtout celles qu'elles peuvent atteindre en sécurité et sans danger Ex : des ressources et des structures qui sont accessibles à pieds et qui fournissent une sécurité personnelle.

Un processus de conception par étapes d'un programme de développement des compétences. Ce processus est adaptatif, flexible et destiné à atteindre les AJF qui ont le plus besoin du programme.

Le nombre et types d'AJF " atteintes " par votre programme.

Un lieu privilégié où les AJF (surtout celles qui sont vulnérables au mariage et aux grossesses précoces) sont accueillies pour les séances modulaires d'éducation non-formelle dirigées par les mentores.

L'idée que les personnes les plus exposées aux pires conséquences ne bénéficient souvent pas des programmes du secteur social en raison de leur isolement et de leur marginalisation.

La répartition de votre population cible sur la base d'expériences et de caractéristiques communes.

Un groupe d'AJF qui ont des expériences et caractéristiques en commun.

La mise en œuvre d'activités connexes au sein des mêmes communautés pour accroître les avantages et gagner en efficacité.

Un programme ou une approche est transformative en matière de genre quand la promotion de l'égalité des sexes - le contrôle partagé des ressources et de la prise de décision - et l'autonomisation des femmes sont au cœur d'une intervention. 
RÉSUMÉ EXÉCUTIF 
Les programmes centrés sur les divers besoins des adolescentes et jeunes femmes (AJF) et qui s'attaquent aux multiples déterminants sociaux du risque (tels que l'isolement social et la déscolarisation) peuvent donner aux AJF les moyens de prendre des décisions et d'influer positivement sur des résultats importants pour elles-mêmes, leurs familles et leurs communautés.

Dans le projet SWEDD, les espaces sûrs sont utilisés pour développer les compétences de vie et remédier aux risques encourus par les AJF qui sont difficiles à atteindre par des canaux de prestation de services formels tels que les écoles et les services de santé. Ces programmes sont appelés "espace sûr" parce que les participantes se réunissent dans des espaces communautaires que les AJF et les parents perçoivent comme sûrs et privés, ce qui peut réduire les obstacles à la participation et permettre de discuter de questions sensibles. Dans le cadre du SWEDD, les espaces sûrs peuvent être installés dans les écoles ou au sein de la communauté.

Ce guide définit les normes minimales, tirées des preuves et de l'expérience mondiales existantes, relatives à la conception, à la mise en œuvre et au suivi, l'évaluation et l'apprentissage requises pour la réussite des programmes des espaces sûrs. Le contenu de ce guide s'inspire des contributions des organisations et des experts mondiaux en programmation et recherche sur les adolescentes, ainsi que de l'expérience du Population Council et de son vaste travail dans le domaine des "espaces sûrs".

Ce guide aidera les planificateurs et les responsables de la mise en œuvre d'un espace sûr d'appliquer une perspective de transformation en matière de genre à leur travail. II leur permettra également de promouvoir les AJF et de transformer la façon dont leurs familles, leurs communautés et elles-mêmes se perçoivent et voient leur potentiel. De nombreuses approches et normes présentées dans ce guide doivent être adaptées aux réalités et aux contextes locaux.

En plus d'aider à planifier de nouvelles activités, peu importe où vous en êtes dans ce processus, le contenu de ce Guide peut être utilisé aux différentes étapes de la conception, de la mise en œuvre et du suivi évaluation et apprentissage d'un espace sûr.

Dans les pages suivantes, nous présenterons des résumés de cinq éléments essentiels des programmes d'espaces sûrs.

1. Les informations locales actuelles sont des éléments clés pour éclairer la conception intentionnelle de programmes centrés sur les AJF, qui doivent être adaptés à votre population cible et à votre contexte spécifique.

2. Les mentores ont besoin de qualifications, de formation, de soutien et de rémunérations adéquates.

3. Le succès et la valeur perçue des espaces sûrs nécessitent un curriculum adapté au segment, des sessions engageantes et dynamiques, et des liens aux autres ressources communautaires.

4. Les sessions doivent être attrayantes et les activités axées sur des sujets de grand intérêt, y compris la fourniture d'opportunités économiques et d'éducation financière. En outre, les familles des AJF et leur communauté devraient percevoir un avantage à permettre à leurs filles de participer.

5. Un plan de Suivi, Évaluation et Apprentissage (SEA) approprié et réalisable aidera à vérifier que le programme d'espaces sûrs réalise les activités prévues et les objectifs du programme, et pour renforcer la mise en œuvre ; il aidera également à mesurer le succès de votre programme en suivant le type de changements qui se produit dans la vie de vos groupes cibles. 
locales peuvent vous aider à déterminer: les AJF "appropriées " qui bénéficieraient le plus des espaces sûrs; quels types de sous-groupes ou "segments" d'AJF devraient être prioritaires pour le ciblage; et quelles compétences seraient les plus utiles pour elles.

Des informations locales sont essentielles pour recruter les AJF "appropriées" en développant une programmation qui sera pertinente et accessible à leur vie. Les programmes centrés sur les AJF basés sur des informations fiables et spécifiques au contexte ont plus de chance d'atteindre leurs bénéficiaires et leurs objectifs que les programmes basés sur une conception de programme préexistant ou des hypothèses non vérifiées. La conception du programme doit tenir compte des réalités des AJF, de leur hétérogénéité et de leurs besoins particuliers en fonction de leur situation (état civil, scolarité, etc.).

Avec la participation de vos segments d'AJF recrutées, vous pouvez examiner les jours, l'heure, la fréquence, et la durée des rencontres des espaces sûrs. Ceci vous aidera à déterminer le moment le plus approprié pour un accès sécurisé et une participation maximum aux espaces sûrs pour chaque segment d'AJF. Aussi, veillez à ce que la participation à un espace sûr ne crée pas de conflit entre les activités habituelles des AJF et le programme.

Enfin, II est important d'obtenir le soutien des chefs de communauté, des parents et des autres membres de la communauté (en menant des entretiens ou des sessions informatives avec des membres de la communauté). Ces acteurs sont essentiels au succès de tout programme d'espace sûr, car ils sont les gardiens des ressources de la communauté ainsi que les participantes elles-mêmes.

Pour la mise en œuvre des espaces sûrs, les mentores, en tant que principales transmetteuses du contenu du programme, sont essentielles pour le succès des espaces sûrs. II faut s'assurer que les mentores aient des qualifications, une formation, un soutien et une rémunération adéquate.

Un profil réaliste des mentores, qui variera en fonction du contexte, est important pour identifier des candidates mentores solides. L'expérience montre néanmoins que les mentores locales qui viennent de la communauté où elles travaillent en tant que telles accroît leur capacité à comprendre et à répondre aux défis auxquels les AJF sont confrontées et permettre ainsi aux AJF de s'identifier à elles.

La formation n'est pas un événement isolé ; les mentores ont besoin d'un soutien continu, d'un encadrement ainsi que des possibilités d'apprentissage continus. Ceux-ci peuvent être atteints avec l'aide des superviseurs. Le soutien des superviseurs joue un rôle important en renforçant les aspects techniques du contenu d'espace sûr et en comblant les lacunes des mentores en matière de connaissances.

Les mentores doivent également être considérées comme un groupe bénéficiaire du programme et on devrait leur donner ; l'opportunité d'organiser et de créer des groupes d'épargne, des initiatives de renforcement de compétences, et des possibilités de générer des revenus. De plus, fournir une rémunération incitative aux mentores les encourageraient à être dévouées au programme et à fournir un travail de qualité. 
3 Liés à la mise en œuvre, le succès et la valeur perçue des espaces sûrs nécessitent un curriculum adapté au segment, des sessions engageantes et dynamiques, et des liens aux autres ressources communautaires. L'ensemble essentiel des

activités doit être basé sur des preuves et faire partie de la théorie du changement du programme qui lie les activités et les interventions du programme aux résultats et aux objectifs du programme et qui devraient être utiles aux AJF dans leur vie; au-delà des espaces sûrs. Pour s'assurer que les AJF et leur communauté trouvent un programme utile et digne de leur temps, il est important qu'elles le considèrent comme une valeur ajoutée à leur vie et à leur communauté, en créant un contenu significatif adapté à la vie réelle des AJF et leurs familles.

Les AJF, même celles qui vivent dans la même communauté, constituent un groupe diversifié. Les compétences prioritaires varient selon le segment d'AJF. Les filles plus âgées ou les jeunes femmes mariées ayant des bébés pourraient être plus intéressées par la fréquentation d'espaces sûrs si elles proposent un contenu visant à les rendre économiquement autonomes dans leur phase de vie puisqu'elles ont des responsabilités financières. Tandis que les adolescentes scolarisées plus jeunes peuvent vouloir davantage de contenus qui les aident à rester à l'école ou qui leur permettent de s'alphabétiser.

Les méthodes participatives sont nécessaires pour engager et dynamiser les sessions de groupe et pour aider à l'apprentissage. Les jeux et les activités génèrent souvent des discussions qui ne peuvent être obtenues par d'autres méthodes. Aussi, le contenu du programme doit être adapté aux AJF (et mentores) analphabètes.

II y a une limite à ce qui peut être accompli dans un espace sûr sous la direction de la mentore. Elles ne peuvent pas fournir aux AJF toutes les informations et tous les services dont elles pourraient avoir besoin. Les espaces sûrs servent de roues motrices pour connecter les AJF aux services essentiels dans leurs communautés ; la mentore devrait pouvoir faciliter l'accès aux ressources communautaires pour les AJF qui participent dans son espace sûr. À moins que votre programme n'offre lui-même des services de santé, ou des services de soutien en cas de VBG, il conviendra d'établir le contact avec des établissements de santé à proximité dans le cas où vous devrez y envoyer les bénéficiaires de votre programme, et former des mentores pour qu'elles puissent orienter efficacement vers ces services. 
Toujours pour la mise en œuvre, afin d'encourager et de maintenir la

fréquentation, les sessions doivent être attrayantes et les activités axées sur des

sujets de grand intérêt, y compris la fourniture d'opportunités économiques et

d'éducation financière. En outre, les familles des AJF et leur communauté devraient percevoir quelconque avantage à permettre à leurs filles de participer.

L'engagement communautaire et la superposition d'autres activités du SWEDD (par exemple: des clubs de maris/clubs de futurs maris et des activités génératrices de revenu) sont des stratégies importantes pour obtenir l'adhésion et le soutien aux programmes d'espace sûr pour les AJF.

Pour chaque AJF, le développement de ses compétences économiques - telles que l'éducation financière et l'accès à la micro-épargne - ainsi que de son soutien social et de ses compétences pratiques - telles que l'auto-efficacité pour accéder aux services de santé, la capacité de négociation - est plus susceptible de lui donner les moyens de faire face aux menaces et de réduire les risques que de s'attaquer seul à l'un de ces problèmes.

Le soutien de la communauté et des familles aux espaces sûrs est nécessaire pour le succès du programme et pour la fréquentation régulière des AJF. II peut également contribuer à créer un environnement favorable à l'AJF pour qu'elle puisse utiliser dans sa vie quotidienne les compétences et les connaissances acquises dans l'espace sûr. Pour obtenir le soutien de la communauté, les programmes doivent s'assurer qu'ils s'engagent auprès des communautés et offrent des avantages supplémentaires aux autres membres de la communauté. Ceci peut être réalisé par la superposition des activités du SWEDD et les dialogues communautaires et d'autres stratégies d'engagement communautaire.

Aussi, la superposition d'activités connexes au sein des mêmes communautés, comme les clubs de maris et clubs de futurs maris, ancrées dans des espaces sûrs bien soutenus pour les AJF, permettrait d'accroître les avantages et de gagner en efficacité. 
Pour le suivi, l'évaluation et l'apprentissage (SEA) des espaces sûrs, il faut avoir un plan de SEA approprié et de taille correcte pour vous aider à vérifier que votre programme d'espaces sûrs réalise les activités prévues et les objectifs du

programme et pour renforcer la mise en œuvre; il vous aide également à mesurer le succès de votre programme en suivant le type de changements qui se produit dans la vie de vos groupes cibles. Le SEA peut créer une boucle de rétroaction qui peut informer et améliorer la coordination, la conception et la mise en œuvre des espaces sûrs. Un plan SEA approprié et faisable doit être " adapté à l'objectif " du programme, ce qui signifie qu'il ne peut pas être trop ambitieux ou complexe. Assurez-vous de ne collecter que les données de S\&E qui sont adéquates à un apprentissage utile et utilisable pour renforcer la mise en œuvre ; il n'est pas nécessaire de collecter des données qui ne seront jamais analysées ni utilisées. Avant de développer votre planification et vos outils de suivi et d'évaluation, il est important d'identifier qui va créer et qui va_utiliser les outils : à quel moment seront utilisés les outils et qui sera responsable de l'analyse et de la présentation des informations que les outils vont collecter.

Le suivi mesure les activités du programme en informant sur le nombre d'activités réalisées, le lieu, les personnes concernées, la période établie, etc. En outre, les données de suivi peuvent être un outil précieux pour suivre la faisabilité de la mise en œuvre de votre programme, identifier les problèmes potentiels à un stade précoce et aider les gestionnaires à suivre une correction en cours d'exécution afin d'atteindre l'objectif du programme.

L'évaluation est un processus systématique qui tente de déterminer objectivement la pertinence et l'efficacité des activités par rapport aux objectifs à atteindre. Les plans d'évaluation ne doivent pas être laissés pour la fin de votre programme mais devraient être un élément central de la planification et de la conception générale. Concentrez-vous sur les objectifs clés de votre programme. Quelques considérations clés que votre programme devrait envisager à évaluer sont : (1) l'ensemble des compétences de protection que vous voulez que les AJF développent, (2) les étapes intermédiaires qui contribuent à vos résultats (les changements dans la connaissance des thèmes, par exemple, la santé reproductive parmi les participantes), et (3) les changements au niveau communautaire (les AJF sontelles perçues différemment ? Est-ce qu'elles sont plus mobiles dans la communauté ?).

Après avoir identifié ce que vous souhaitez évaluer, il convient de définir les indicateurs " SMART " (Spécifiques, Mesurables, Appropriés, Réalistes, et Limités dans le Temps) que vous utiliserez pour représenter vos résultats ainsi que les moyens pour collecter les données.

Un mécanisme de retour d'information, feedback, doit être intégré dans la structure de gestion du programme pour garantir que les gestionnaires ne manquent pas une occasion d'apprendre. Le feedback doit être basé sur des données de suivi et doit être fourni aux parties prenantes à tous les niveaux (y compris les mentores) de la structure organisationnelle.

La conception et le suivi des programmes pour les AJF devraient être accompagnés d'un " apprentissage ". La meilleure façon de favoriser l'utilisation des données et l'apprentissage est de s'assurer que les gestionnaires et le personnel de mise en œuvre perçoivent la valeur inhérente des données et leur avantage pour le programme et les AJF que vous soutenez. Cela vous permettra d'évaluer en profondeur la conception et la mise en œuvre du programme tout en procédant à des ajustements stratégiques. 
|NTRODUCTION 
BUT : $\quad$ Améliorer la programmation et les pratiques de SWEDD pour augmenter l'efficacité et la rentabilité des activités centrées sur les AJF, y compris pendant la mise à l'échelle.

OBJECTIF : Communiquer clairement et de façon concise les normes minimales pratiques pour la conception, la mise en œuvre et le suivi et évaluation des plateformes communautaires pour les AJF, à savoir les espaces sûrs et les activités SWEDD connexes. Les normes minimales visent à garantir que les AJF " appropriées " sont atteintes, par les interventions " appropriées ", au moment " approprié, " en employant une approche transformatrice au genre qui s'allie à la promotion de l'égalité des genres - le contrôle partagé des ressources et de la prise de décision - et à l'autonomisation des femmes. Ces normes minimales sont utiles pour tous les pays SWEDD, mais ce guide inclut également des discussions de certains aspects qui devront être adaptés à certains contextes spécifiques.

APPROCHE : L'élaboration participative de ce guide a été dirigée par le Population Council avec l'apport du Secrétariat Technique Régionale de I'UNFPA, les représentants nationaux UNFPA, les membres des unités de gestion de projets (UGP) SWEDD des pays concernés.

PUBLIC : $\quad$ Partenaires de mise en œuvre du SWEDD et le personnel du SWEDD responsable des espaces sûrs et des autres activités centrées sur les AJF.

COMMENT : Ce guide peut être utilisé à n'importe quel stade de la mise en œuvre. Le contenu est conçu pour être informatif et exploitable par les équipes des pays SWEDD à différentes phases de programmation, surtout lorsqu'il s'agit d'élargir la couverture.

QUOI : Le contenu de ce guide est tiré des expériences de SWEDD I, du Population Council et d'autres organismes dans le monde entier, ainsi que des données disponibles sur les espaces sûrs et les activités connexes (centrées sur les AJF).

POURQUOI: Des décennies d'expérience ont montré qu'on doit adopter des approches pour identifier et atteindre les adolescentes (des AJF et jeunes femmes âgées de 10 à 19 ans ${ }^{1}$ ) les plus vulnérables. Certaines AJF qui se trouvent à l'intersection de normes de genre inéquitables, de la sexualité émergente et de la jeunesse sont particulièrement vulnérables en raison de : l'isolement social, l'absence de scolarisation pendant l'adolescence, le mariage précoce, les pressions exercées pour fournir un travail productif et un revenu, entre autres. Au sein de la population des AJF, il existe des sous-ensembles de AJF, ou "segments" ayant des expériences et des circonstances de vie différentes (scolarisation, état civil, etc.) qui font que leurs besoins, leurs défis et leurs solutions sont différents de ceux des AJF ayant des circonstances différentes. Une approche spécifique à chaque segment est nécessaire et doit tenir compte de l'hétérogénéité des AJF.

Lorsque les AJF expriment leur voix, leur choix ou exercent leur contrôle, elles peuvent commencer à perturber les normes sociales. Au sein de familles, de communautés et d'environnements politiques favorables, cela peut améliorer la santé, l'éducation et les moyens de subsistance des AJF. Et si de nombreuses AJF dans une communauté donnée participent à des programmes d'autonomisation, cela peut créer un "point de basculement" qui favorise de nouvelles normes sur la valeur des AJF qui perdurent. En fin de compte, ces changements amélioreront considérablement le bien-être des AJF dans la vie et favoriseront l'égalité des sexes. ${ }^{2}$

\footnotetext{
1 “Les adolescents sont définis par les Nations Unies comme les personnes âgées de 10 à 19 ans" UNICEF 2012. "progrès pour les enfants Un bilan sur les adolescents Numéro 10, avril 2012".

"Adolescent: 10-19 ans" OOAS, 2016. "ANALYSE SITUATIONNELLE de la sante des adolescents et jeunes y compris la sante sexuelle et reproductive dans l'espace CEDEAO"

2 Temin, M., Amin, S., Ngo, TD., Psaki, S. “How to Give Adolescent Girls Voice, Choice, and Control; a

Framework for programs focused on empowering adolescent girls." 2018. Stanford Social Innovation

Review. https://ssir.org/articles/entry/how to give adolescent girls voice choice and control
} 
En 2019, pour célébrer la Journée internationale de la fille, des experts de 20 organisations, dont des chercheurs, des responsables de la mise en œuvre de programmes, des experts en politiques et des donateurs, se sont réunis pour relever le défi suivant : malgré des efforts accrus et des données probantes concernant les adolescentes, les investissements ne sont pas suffisants pour atteindre les filles marginalisées à risque.

L'atelier a permis aux expertes réunies de partager les bonnes pratiques, les leçons tirées des erreurs et les preuves, d'apprendre les unes des autres et de co-créer.

\section{Comment ce guide est-il structuré ? Comment dois-je l'utiliser pour éclairer la conception et la mise en œuvre de mon programme et du SEA ?}

Dans ce document, nous nous efforçons de présenter les informations importantes d'une manière accessible et facile à naviguer. Cela inclut la mise en évidence des messages clés, la présentation des choses à faire et à ne pas faire, et des études de cas.

Ce guide a pour but de vous faire passer en revue les différentes considérations qui auront un impact sur les trois étapes principales:

1. La conception du programme,

2. La mise en œuvre, et

3. Le suivi, l'évaluation et l'apprentissage.

Bien que le contenu soit présenté en trois "étapes", l'intention est de se référer à ce guide et de I'utiliser pour améliorer chaque aspect des espaces sûrs tout au long de la durée de vie du projet. II ne s'agit pas d'un processus unique. Les informations recueillies lors de l'étape 3, suivi, évaluation et apprentissage, doivent être utilisées pour guider les ajustements à la conception et à la mise en ceuvre de votre programme afin de renforcer votre programme et d'avoir un impact plus important sur les participants.

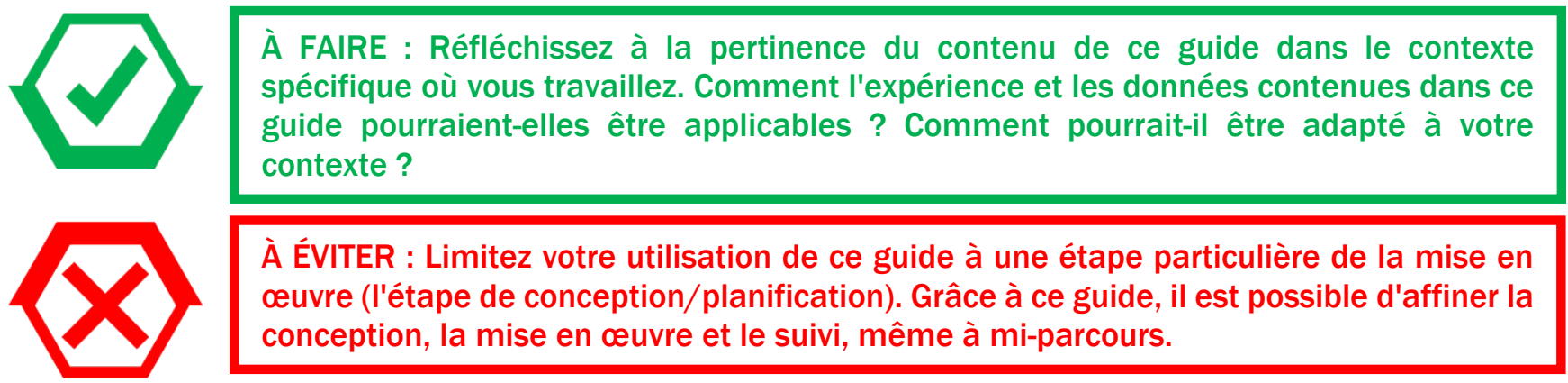

\section{Pourquoi les AJF ont-t-elles besoin d'un "espace sûr"?}

Un espace sûr est un lieu privilégié où les AJF (en particulier celles qui sont vulnérables au mariage et aux grossesses précoces), sont accueillies pour les séances modulaires d'éducation nonformelle dirigées par les mentores. C'est un lieu où les AJF se sentent physiquement et émotionnellement en " sécurité " leur permettant de renforcer leur réseau de soutien social, d'être à l'aise. C'est un lieu où elles expriment librement leurs pensées et leurs sentiments, même les sujets les plus sensibles, sans crainte ni jugement, et où elles peuvent s'épanouir avec leurs pairs. 


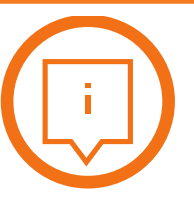

Les programmes centrés sur les divers besoins de l'AJF et qui s'attaquent aux multiples déterminants du risque (l'isolement social et la déscolarisation) donnent aux AJF les moyens de prendre des décisions et d'influer positivement sur des résultats importants pour elles-mêmes, leurs familles et leurs communautés.

\section{Type d'espace sûr dans le projet SWEDD}

Dans le projet SWEDD, à chaque cohorte, l'espace sûr accueille des AJF en deux tranches d'âge : la première inclut des AJF âgées de 10 à 14 ans et la seconde est constituée des AJF âgées de 15 à 19 ans. Les bénéficiaires des espaces sûrs sont réparties en des groupes de 25 membres en moyenne. Leur participation dans ces espaces sûrs leur permet de développer des compétences de vie. Ceci est important vu que la plupart des adolescentes de 10 à 19 ans vivant dans des pays à faible et moyen revenus sont bien placées pour développer des capacités, réaliser leur potentiel et contribuer à la santé et au bien-être de leur famille et de leur communauté.

Les espaces sûrs sont utilisés pour remédier aux risques encourus par les AJF qui sont difficiles à atteindre par des canaux de prestation formels tels que les écoles et les services de santé. Ces programmes sont appelés "espace sûr" car ils se réunissent dans des espaces communautaires que les AJF et les parents perçoivent comme sûrs et privés, réduisant ainsi les obstacles à la participation et leur permettant de discuter de questions sensibles. Les espaces sûrs peuvent être installés dans les écoles ou au sein de la communauté.

Dans le projet SWEDD, ceux qui sont dans les écoles sont placés sous l'autorité du Ministère en charge de l'Éducation nationale, les bénéficiaires sont les adolescentes à risque d'abandon scolaire. Les espaces sûrs relevant de la communauté sont placés sous l'autorité du Ministère en charge du genre ; les bénéficiaires sont les adolescentes et les jeunes AJF n'ayant pas eu la chance d'avoir accès à l'école, ou sont des déscolarisées, confrontées à la précarité, à la vulnérabilité et sont prédisposées à être mariées avant d'atteindre l'âge de 18 ans. Une priorité est donnée aux adolescentes de 10 à 14 ans et aux orphelines.

Dans ces groupes, les AJF rencontrent régulièrement un responsable (par exemple, un mentor) qui utilise diverses méthodes pédagogiques pour aborder la santé sexuelle et reproductive (SSR), la prévention du $\mathrm{VIH}$, les compétences de vie courante, l'autonomisation économique et financier, et d'autres sujets.

La gestion des espaces sûrs est confiée à une ONG ou à une représentante d'un ministère (e.g. le ministère de la famille ou de l'éducation) choisie en fonction de ses compétences et de son expérience d'intervention dans la région d'implantation des espaces sûrs.

\section{Paquet d'activités minimum dans un espace sûr}

La stratégie des espaces sûrs repose sur une intervention tridimensionnelle (sur la fille, sur la communauté et sur les institutions) qui vise la réduction des mariages précoces en les retardant à l'âge minimum de 18 ans, la réduction des grossesses précoces des adolescentes et le recours à la planification familiale (Figure 1). 
Figure 1 : Approche modélisée du paquet intégré de services qu'est l'espace sûr

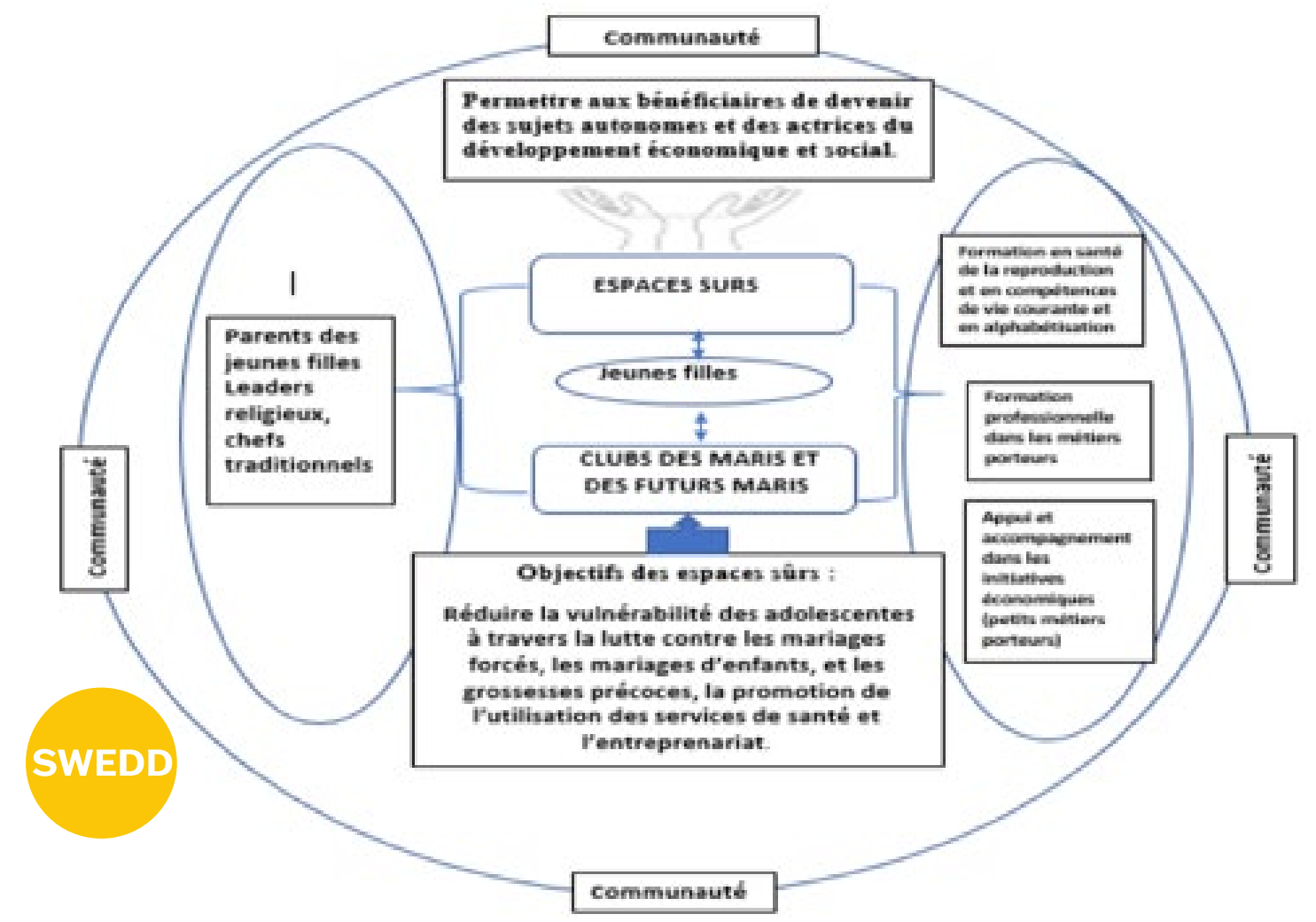

Ainsi, les axes d'intervention comprennent les sessions éducatives, les séances d'alphabétisation, les visites à domicile, les activités de formation professionnelle ou d'accompagnement des initiatives économiques, l'engagement communautaire et, dans certains pays, d'autres activités complémentaires pour les jeunes femmes ou pour les garçons adolescents. Pour plus de détails sur le contenu approprié pour les espaces sûrs, veuillez-vous référer à la section : Un contenu programmatique approprié pour des espaces sûrs afin de développer des "compétences" et des aptitudes à la vie quotidienne pertinentes (Étape 2, page 38).

\section{Ressources}

Rassembler à ce niveau les ressources humaines, matérielles, et financières nécessaires (Pour plus de détails, consulter la section sur les considérations relatives au coût (page 22).

1. Ressources d'apprentissage dans les espaces sûrs : outils adaptés aux modules tels que la contraception, les serviettes hygiéniques pour faire des démonstrations.

2. Ressources matérielles pour le fonctionnement des espaces sûrs : nattes, décorations, tableau, craie, chiffon, container pour l'eau potable.

3. Ressources espaces sûrs pour les mentores et les superviseurs: les tablettes pour enregistrer la présence des AJF, les modules dispensés, le suivi par les superviseurs.

4. Ressources pour les AJF : collations/snacks, jeux à leur intention, livres, (qu'elles peuvent utiliser pendant les temps libres même après les heures de rencontre des espaces sûrs). 


\section{ÉTAPE 1 : CONCEPTION INTENTIONNELLE; \\ LA CONCEPTION ET LA PLANIFICATION DES PROGRAMMES}




\section{ÉTAPE 1: CONCEPTION INTENTIONNELLE ; LA CONCEPTION ET LA PLANIFICATION DES PROGRAMMES}

Ce chapitre se concentre sur les détails à considérer lors de la conception d'un programme centré sur les AJF. II est essentiel d'être intentionnel ${ }^{3}$ dans la conception afin d'augmenter la probabilité que les interventions soient mises en œuvre avec qualité et efficaces et qu'elles atteignent les objectifs visés. La conception du programme doit tenir compte des réalités et des expériences vécues par les AJF, de leur hétérogénéité et de leurs besoins particuliers en fonction de leur situation (état civil, scolarité, etc.).

Investir dans les AJF, plus particulièrement celles exclues des communautés les plus pauvres, améliore la santé, réduit la fécondité, augmente les niveaux d'éducation et de compétences. De même, il crée une base pour des moyens de subsistance décents et des économies locales plus fortes, et introduit des pratiques environnementales durables dans les foyers, les communautés et dans la gestion globale des ressources ${ }^{4}$.

Résumé de la conception du programme :

- Qui : établir les critères optimaux de ciblage et de recrutement.

- Où : normes pour impliquer les communautés et créer un environnement propice aux espaces sûrs.

- Quoi : considérations pour passer à la conception et la planification du programme à l'échelle.

\section{Une programmation conçue intentionnellement avec les AJF au centre}

L'approche de la conception intentionnelle ${ }^{5}$ est un processus de conception par étapes d'un programme de développement des compétences utilisé pour les programmes centrés sur les AJF.

II est essentiel pour l'approche de Développement des Compétences de Protection (voir page 39 pour la définition des compétences de protection) de prendre des décisions de programme (sur la conception, la mise en œuvre et le suivi) basées sur des informations locales. L'expérience du Population Council confirme que les programmes centrés sur les AJF basés

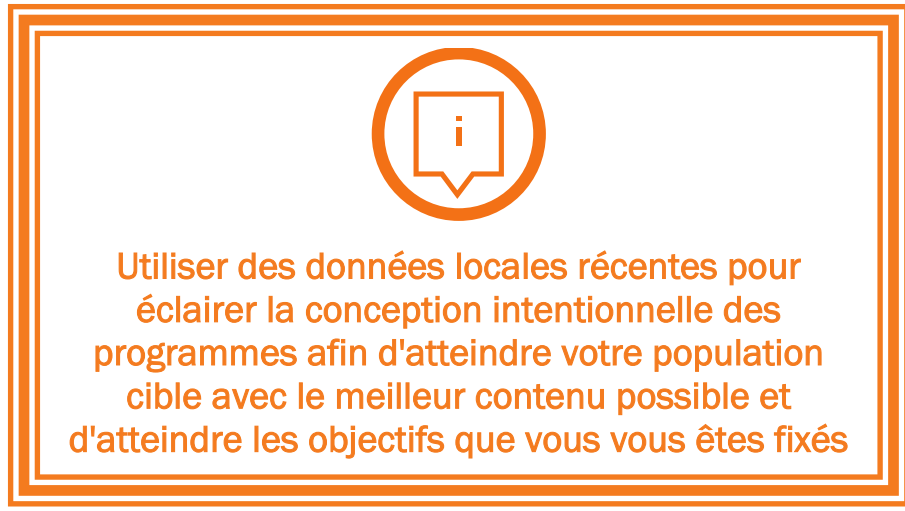
sur des informations fiables et spécifiques au contexte ont plus de chance d'atteindre leurs bénéficiaires et leurs objectifs que

\footnotetext{
3 Cela signifie qu'il faut prendre des décisions explicites à chaque étape critique de la planification du programme, en se basant sur des preuves et des informations locales

${ }^{4}$ Adapté de : Intentional Design : Reaching the Most Excluded Girls in the Poorest Communities : A Guide for Practitioners and Advocates, Population Council

${ }^{5}$ Adapté de : Adolescent Girls Community of Practice | About| What is Intentional Design ?, Population Council
} 
les programmes basés sur une conception de programme préexistant ou sur des hypothèses non testées.

Un certain nombre d'étapes peuvent être utiles pour soutenir l'apprentissage durant la planification, la mise en œuvre et le suivi d'un programme fondé sur des faits. Ces mesures sont résumées dans le Processus de conception par étapes d'un programme de développement des compétences. Ce processus de conception peut permettre d'intégrer les concepts de l'Approche de développement des compétences de protection dans votre programme centré sur les AJF. 6

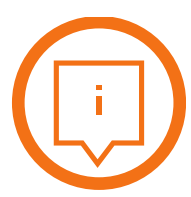

Peu importe où vous en êtes dans ce processus, il est possible d'améliorer les étapes que vous répétez; vous pouvez toujours utiliser ce qui a été acquis lors d'une étape pour informer des autres étapes du processus.

\section{DES NORMES MINIMALES POUR UN CIBLAGE ET UNE COUVERTURE OPTIMALE}

\section{Une programmation qui cible et atteint les AJF appropriées}

Bien que les bénéficiaires des espaces sûrs aient parfois le même âge (ex 15 ans), elles sont socialement distinctes ; leurs vies contrastent à bien des égards, notamment en ce qui concerne les pressions quotidiennes, les endroits où elles se sentent à l'aise et les services auxquels elles peuvent accéder en toute sécurité. Ainsi, un groupe de pairs n'est pas simplement constitué d'individus qui partagent un même âge et un même sexe, mais d'individus qui ont également des liens significatifs entre eux grâce à une expérience partagée ou similaire.

Les AJF sont plus à l'aise avec celles qui ont des expériences de vie similaires et qui sont du même âge. Les programmes pour les pairs permettent de mettre en place des plans de recrutement solides, des accords de mentorat et de développer le contenu, se traduisant généralement par une plus grande participation et une meilleure rétention dans les programmes. En bref, ces programmes ne sont pas simplement centrés sur les AJF, ils sont centrés sur les pairs.

Pour avoir un impact durable, il est essentiel que les programmes centrés sur les AJF atteignent les AJF "appropriées." Si les AJF participantes au programme sont plus nombreuses à être confrontées à un risque élevé de mariage d'enfants, de grossesse précoce, d'abandon scolaire et autres, il est plus probable que le programme aura les effets souhaités. Plutôt que de traiter les AJF et les jeunes femmes comme un groupe homogène, il est important de comprendre les risques et les opportunités uniques auxquels sont confrontées les différentes sous-populations ou

${ }^{6}$ Adapté de : $\underline{\text { https://www.popcouncil.org/uploads/pdfs/2016PGY_GirlsProtectiveAssetsTools_fr.pdf }}$ 
segments ${ }^{7}$ d'AJF et d'adapter le matériel du programme en conséquence. Le tableau 1 démontre un exemple d'une segmentation possible pour des AJF en utilisant des exemples de caractéristiques.

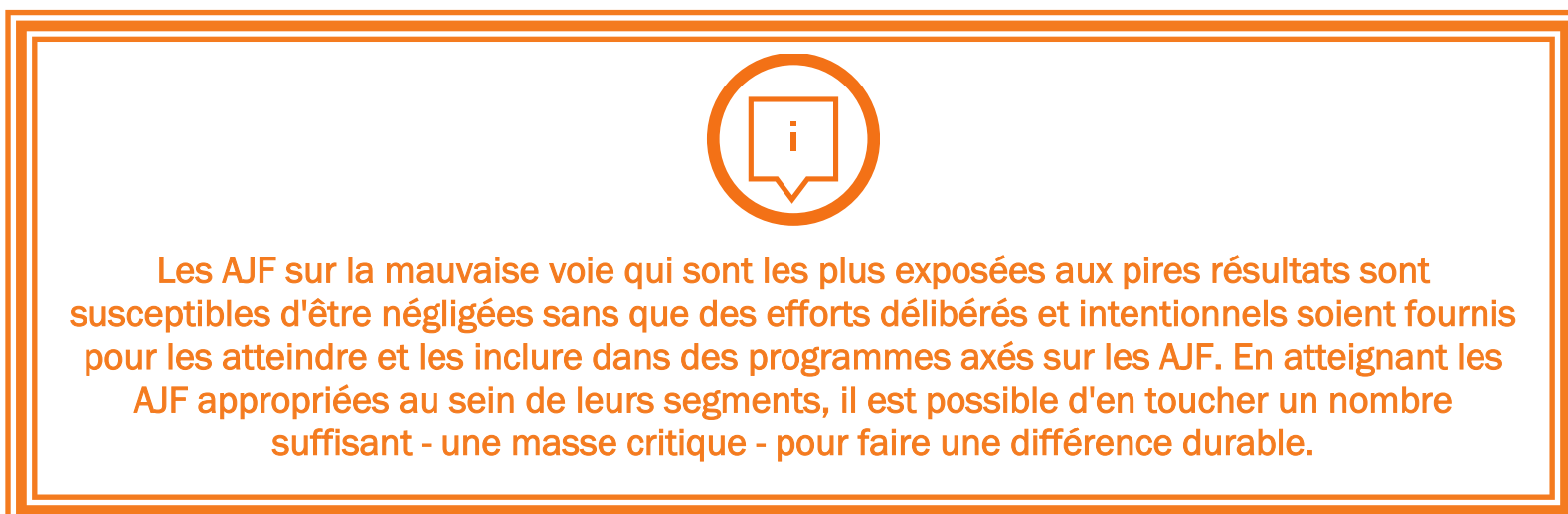

Tableau 1. Un exemple d'une segmentation des AJF pour les programmes utilisant des indicateurs sur l'éducation et le mariage

\begin{tabular}{|c|c|c|c|}
\hline \multirow{2}{*}{ Segment d'AJF } & \multicolumn{2}{|c|}{ Indicateur de l'éducation } & \multirow{2}{*}{$\begin{array}{l}\text { Indicateur de } \\
\text { mariage }\end{array}$} \\
\hline & Statut scolaire & Le niveau scolaire & \\
\hline $\begin{array}{l}\text { AJF " relativement " } \\
\text { avantagées }\end{array}$ & À l'école & Niveau secondaire & Jamais mariées \\
\hline $\begin{array}{l}\text { AJF " ordinaires " ou } \\
\text { moyennes }\end{array}$ & À l'école & Niveau primaire & Jamais mariées \\
\hline AJF vulnérables & $\begin{array}{l}\text { Ne sont jamais } \\
\text { allées à l'école ou } \\
\text { ne sont pas } \\
\text { scolarisées }\end{array}$ & $\begin{array}{l}\text { Pas d'école ou } \\
\text { quelques années de } \\
\text { scolarité }\end{array}$ & Jamais mariées \\
\hline AJF les plus vulnérables & $\begin{array}{l}\text { Ne sont jamais } \\
\text { allées à l'école ou } \\
\text { ne sont pas } \\
\text { scolarisées }\end{array}$ & Pas d'école & Mariées \\
\hline
\end{tabular}

${ }^{7}$ Cela signifie un groupe d'AJF qui ont des expériences et caractéristiques en commun.

Ex : jeunes mères, adolescentes, mariées, et non-scolarisées 
À FAIRE : Réfléchir à certaines des différences entre les AJF et à la manière dont leurs expériences et situations peuvent affecter leurs opportunités et leur potentiel (ex. AJF handicapées, AJF réfugiées ou déplacées, adolescentes mariées, orphelines, AJF migrantes vivant en dehors de leur famille, jeunes mères, AJF nomades/très mobiles, etc.)

À ÉVITER : Croire que toutes les adolescentes sont les mêmes et que les problèmes auxquels elles sont confrontées et le soutien dont elles ont besoin seront les mêmes. 
Comment identifier des segments des AJF les plus vulnérables, qui bénéficieront le plus de notre programme?

En général, vous avez besoin d'informations locales en temps réel pour compléter les statistiques nationales et infranationales (régionales) disponibles qui ne sont pas suffisamment désagrégées ou détaillées pour comprendre les ressources et les obstacles dans les communautés du point de vue des AJF et des jeunes femmes. Ces informations sont essentielles pour recruter les AJF " appropriées " et en développant une programmation qui sera pertinente et accessible à leur vie, elles peuvent être collectées à l'aide de techniques numériques ou à l'aide d'un crayon et d'un papier.

Un moyen de collecter ces informations locales est l'outil numérique pratique du Population Council, "Le Girl RosterTM ".

Le Girl Roster (Le registre des AJF) peut aider à comprendre la communauté quelles AJF vivent dans la communauté de programme -et obtenir des informations fondamentales pour relier intentionnellement les AJF - en particulier les adolescentes les plus marginalisées - aux ressources, aux installations et aux services vitaux auxquels elles ont droit, mais souvent ont un accès limité ou inexistant.

Le Girl Roster élaboré par le Population Council et ses partenaires est un outil

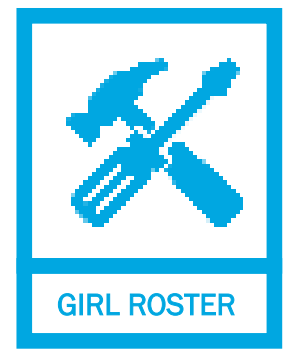
pratique pour la conception des programmes facilitant l'accès des AJF aux ressources vitales, aux structures et aux services. Il est composé de deux parties :

1. Un questionnaire destiné aux ménages qui donne une idée du nombre de AJF présentes dans la zone d'intervention du programme, et

2. Un outil d'analyse rapide qui catégorise les AJF par âge, scolarité, état civil, grossesse ou modalité de vie.

L'outil est utile pour les chargé(e)s de programmes qui commencent à travailler dans une nouvelle région et pour celles et ceux qui veulent évaluer et améliorer leur couverture ${ }^{8}$ actuelle. L'outil aide à : (1) reconnaître les différents sous-groupes de AJF (univers complet) dans une zone spécifique du programme ; et (2) améliorer l'accès des AJF à une part équitable des ressources communautaires, grâce à un engagement communautaire adéquat.

Remarque : Le Girl Roster n'a pas pour objectif d'être un outil de recherche, de suivi ou d'évaluation.

${ }^{8}$ Cela signifie le nombre et types d'AJF " atteintes " par votre programme 
L'outil peut aider les programmes à identifier les AJF les plus vulnérables et à apporter des changements mesurables dans leur vie, augmentant ainsi l'efficacité du programme. L'outil a été développé pour réduire l'exclusion, tout en reconnaissant que même avec les meilleurs intentions, certains programmes pour AJF n'appliquent pas des pratiques fondées sur des données probantes pour mieux aider ces dernières, et finissent dès lors par recruter moins d'AJF que ce qu'ils pourraient. L'expérience montre constamment que certains programmes " captent l'élite " où la majorité des avantages vont aux AJF qui sont relativement " sur la bonne voie " pour accomplir une transition en sécurité vers l'âge adulte, et pas celles qui sont " marginalisées ". 9

Des informations locales actualisées permettent de bien comprendre la communauté dans laquelle nous travaillons et les AJF qui y vivent. Avec une image claire de la population des AJF dans la communauté, vous pouvez définir des segments prioritaires d'AJF et planifier intentionnellement pour atteindre, recruter et retenir les plus vulnérables d'entre elles.

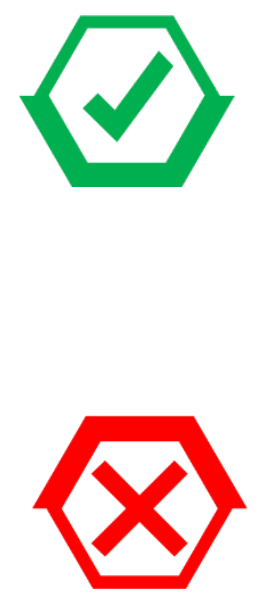

À FAIRE : Réfléchir de manière critique aux AJF qui sont les plus vulnérables et utilisez des informations actuelles et spécifiques au contexte pour identifier et atteindre les adolescentes les plus vulnérables.

Analysez systématiquement la couverture, y compris les détails sur les participantes, afin de déterminer si le groupe cible participe au niveau attendu.

À ÉVITER : "L'inversion des avantages " ou "l'accaparement des ressources par les élites " c'est-à-dire ceux/celles qui commencent par les plus grands avantages typiquement plus âgé(e)s, les enfants scolarisé(e)s - reçoivent plus de ressources ; ceux qui commencent avec le moins d'avantages (plus jeunes, les AJF non scolarisées) reçoivent moins de ressources.

\section{Plans intentionnels pour éviter "l'accaparement des ressources par les élites" et "l'inversion des avantages"}

Les participantes attirées par les activités et qui sont en mesure d'y accéder (physiquement, financièrement et socialement) sont plus susceptibles de participer. Ce sont probablement les AJF dans la communauté relativement avantagées (à l'école, jamais mariés, etc.) et moins exposées au mariage des enfants, aux grossesses précoces, au décrochage scolaire que les autres AJF.

Les AJF les plus exposées aux pires conséquences, comme le mariage des enfants, les grossesses précoces et l'infection par le $\mathrm{VIH}$, ne bénéficient souvent pas des programmes du secteur social

\footnotetext{
${ }_{9}$ Adapté de " Développer les compétences de protection des AJF: Ensemble d'Outils pour la Conception d'un Programme " du Population Council
} 
en raison de leur isolement et de leur marginalisation. Ce concept est souvent appelé "l'inversion des avantages" (Figure 2). Cette figure illustre que les AJF les plus vulnérables ont des besoins plus importants, mais qu'elles bénéficient le moins des programmes typiques tandis que les AJF qui sont relativement plus avantagées souvent reçoivent le plus grand bénéfice des programmes.

Figure 2. Distribution typique de programme : l'inversion des avantages

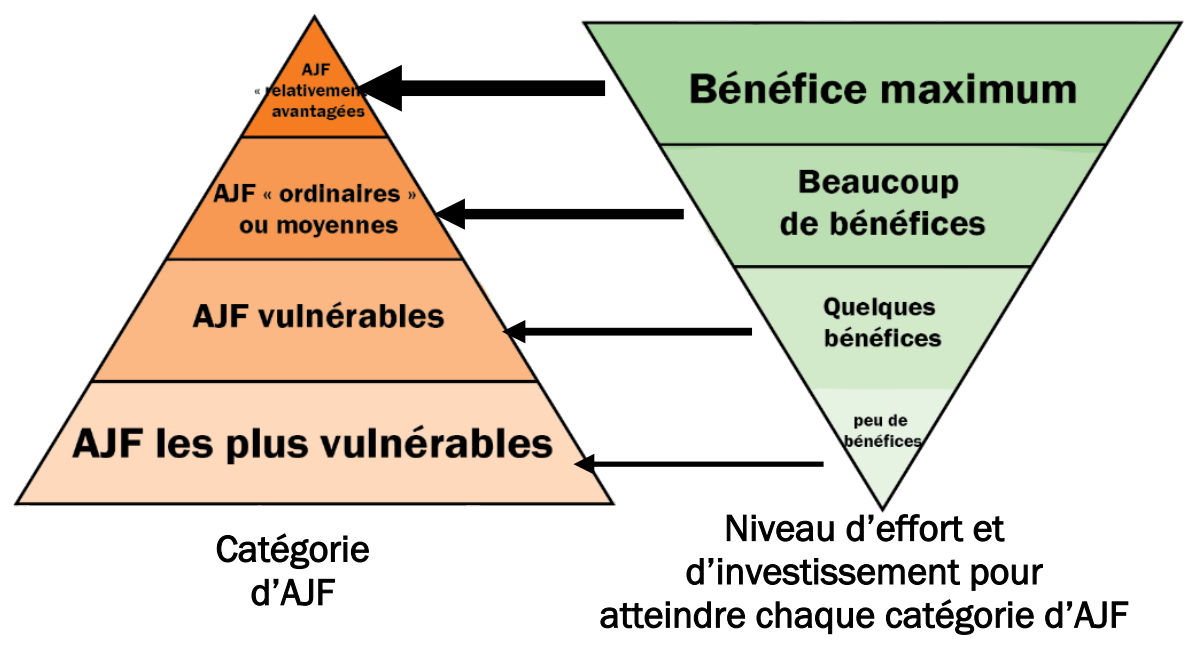

Les AJF n'ayant pas de contact avec les écoles où se déroulent souvent les programmes destinés aux jeunes peuvent également être exclues des services officiels de santé et financiers et des marchés du travail. Et malheureusement, les adolescentes qui ont accès aux établissements de santé reçoivent rarement des services adaptés aux adolescents ; les prestataires peuvent négliger leurs besoins spécifiques en matière de santé ou les traiter avec insensibilité ${ }^{10}$.

" De nombreux programmes " au service des jeunes" avec les meilleures intentions servent avant tout celles qui sont les plus privilégiées et manquent les segments des jeunes ayant le plus besoin de services et de soutien. Cette " inversion des avantages " peut être le résultat d'un recrutement basé sur la demande, qui attire les jeunes déjà mobiles et capables d'affirmer leur pouvoir. Des étapes intentionnelles doivent être suivies pour recruter celles qui sont le moins en mesure d'exprimer leur souhait de participer. L'organisation d'une réunion des parents pour expliquer le programme et avoir leur approbation risquerait d'exclure les AJF qui n'ont pas de parents, ou celles dont les parents n'approuvent pas leur participation, ou encore celles dont les parents n'étaient pas au courant de la réunion ou ne pouvaient pas y assister. Le recrutement par les écoles passe à côté des AJF qui ne sont pas scolarisées." 11

10 World Health Organization (WHO). Making Health Services Adolescent Friendly: Developing National Quality Standards for Adolescent Friendly Health Services. Geneva: WHO; 2012. Accessed May 19, 2020. https://www.who.int/maternal_child_adolescent/documents/adolescent_friendly_services/en/ 11 Population Council. (2016) “Développer les compétences de protection des AJF: Ensemble d’Outils pour la Conception d'un Programme. " https://www.popcouncil.org/uploads/pdfs/2016PGY GirlsProtectiveAssetsTools fr.pdf dans Temin and Heck. " Close to Home : Evidence on the Impact of Community-Based Girl Groups 


\section{(1)}

Pour les programmes qui ciblent les AJF et jeunes femmes et qui visent à améliorer la santé, l'éducation et l'autonomisation des AJF-comme le SWEDD-cette inversion des avantages se produit souvent, sans le vouloir. Cela peut signifier que les AJF les plus pauvres et les plus vulnérables qui courent le plus grand risque des pires résultats (niveau bas d'éducation, mariage précoce, grossesse précoce, taux élevé de fécondité) sont exclues non-intentionnellement des activités, entravant ainsi le progrès. Des efforts intentionnels sont nécessaires pour atteindre et inclure les AJF les plus à risque.

\section{DES NORMES MINIMALES POUR L'ENGAGEMENT COMMUNAUTAIRE :}

Après avoir établi clairement votre cible, l'étape suivante portant sur la conception consiste à cerner les besoins des AJF ciblées et la communauté dans laquelle elles vivent, ensuite de comprendre comment les caractéristiques structurelles et sociales d'une communauté de AJF jouent un rôle essentiel dans la détermination des effets que les programmes peuvent obtenir.

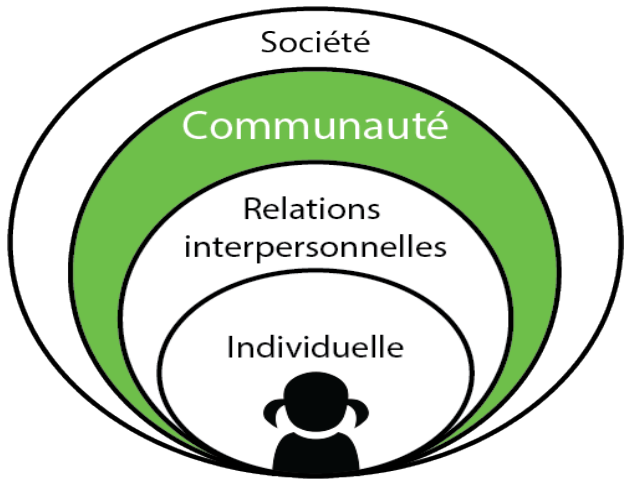

Les communautés de AJF comprennent à la fois les espaces physiques dans lesquels elles doivent se déplacer mais aussi l'ensemble des adultes et des pairs qui façonnent leurs croyances sur le mariage, le travail et l'éducation. En d'autres termes, la communauté participe activement au programme, et son influence sur les programmes destinés aux AJF est tout aussi importante que celle des mentores, du personnel du programme et des autres personnes qui ont un rôle officiel dans la mise en œuvre. 12

Comprendre l'environnement opérationnel afin d'éclairer la conception d'une programmation centrée sur les AJF

Il faut comprendre quelles ressources existent pour les AJF dans la communauté et qui y a accès. Ces informations sur la communauté locale nous permettront de voir et de comprendre les risques, les opportunités, les obstacles et les ressources qui existent dans la communauté pour les AJF de leur propre point de vue plutôt que des suppositions. Par exemple : ce qu'une AJF perçoit comme étant un endroit risqué ou dangereux dans sa communauté peut différer de ce qu'un membre masculin du personnel du projet (qui prend les décisions concernant le lieu des réunions dans un espace sûr) percevrait comme un endroit dangereux ou risqué.

12 Adapté de: "More than a Backdrop"

https://www.popcouncil.org/uploads/pdfs/2019PGY_CommunityActionGuide.pdf du Population Council 
Où trouver ces informations :

- Des entretiens avec

- Des parents,

- Des enseignants,

- Des employés,

- Des ONG,

- Des leaders féminins,

- Des leaders communautaires, et

- Des prestataires de soins de santé.

- Les exercices qui font participer les AJF, tels que la cartographie physique (ex. la page 18) et sociale pour identifier les ressources et les activités auxquelles elles participent ou non, et pourquoi, sont instructifs.

- Les informations sur les personnes de confiance sur lesquelles les AJF comptent au sein de la communauté peuvent apporter une valeur ajoutée.

Des consultations permanentes avec les membres de ces groupes peuvent contribuer à instaurer la confiance et apporter des nuances importantes aux programmes. 13

\section{Une conception de programme ancrée dans le propre point de vue de l'AJF}

Pour comprendre les réalités des AJF, l'équipe du programme doit se concentrer sur l'accès réel des AJF aux ressources au sein de leur "communauté accessible à pied " ", autrement dit des ressources et des structures auxquelles elles ont accès à pied et qui leur fournissent une sécurité personnelle en leur permettant d'exprimer leur pouvoir. Leur " communauté " devrait être définie non seulement par leurs frontières physiques, mais aussi par les personnes et les ressources qui déterminent leur espace social.

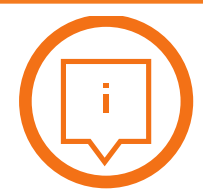

Examinez les jours, l'heure, la fréquence, et la durée possibles des réunions des espaces sûrs avec vos segments d'AJF recrutées afin de déterminer le moment le plus approprié pour un accès sécurisé et une participation maximum aux espaces sûrs pour chaque segment d'AJF et chaque contexte. 14

En ce qui concerne les segments cibles, les jours, les heures et la fréquence des sessions des espaces sûrs peuvent potentiellement rendre les réunions plus ou moins accessibles, ce qui fait une réelle différence sur le long terme.

Lors de l'examen de vos segments cibles, le choix du lieu idéal pour vos espaces sûrs communautaires est vital pour la réussite du programme. La figure 3 ci-dessous présente deux fiches de travail potentiellement utiles pour identifier ces détails logistiques, dans une communauté, qui sont essentielles de comprendre pour un espace sûr réussi et bien fréquenté.

13 Blake, Sarah and Miriam Temin. 2019. "Understanding the Role of Communities in Programming for Adolescent Girls: More Than a Backdrop." New York: Population Council. https://www.popcouncil.org/uploads/pdfs/2019PGY CommunityActionGuide.pdf 14 Adapté de https://www.popcouncil.org/uploads/pdfs/2016PGY_GirlsProtectiveAssetsTools.pdf 
Figure 3. Feuilles de Travail : Timing, fréquence et durées des réunions et Lieux Appropriés ${ }^{15}$

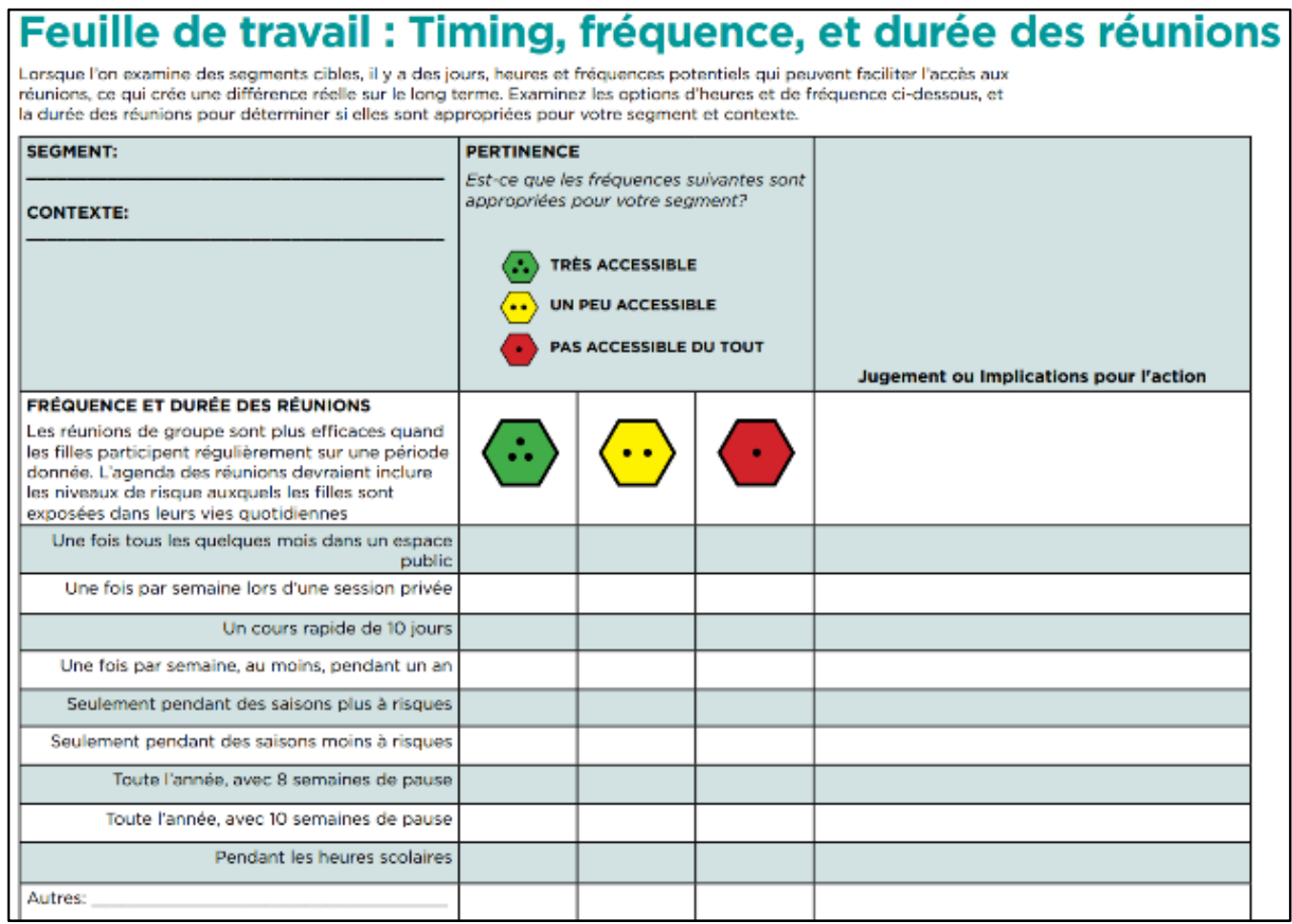

\section{Feuille de Travail : Lieux Appropriés}

Lors de l'examen de vos segments cibles, le choix du lieu idéal pour vos plates-formes communautaires de filles est vital

pour la reussite du programme. En plus de s'assurer que le lieu est sor, privé et régul erement accessible aux filles et aux

jeunes femmes, il doit aussi etre acceptable pour les parents et les membres de la communaute. Considerez les options de

lieux et de determiner leur pertinence compte tenu de votre segment et le cadre du programme.

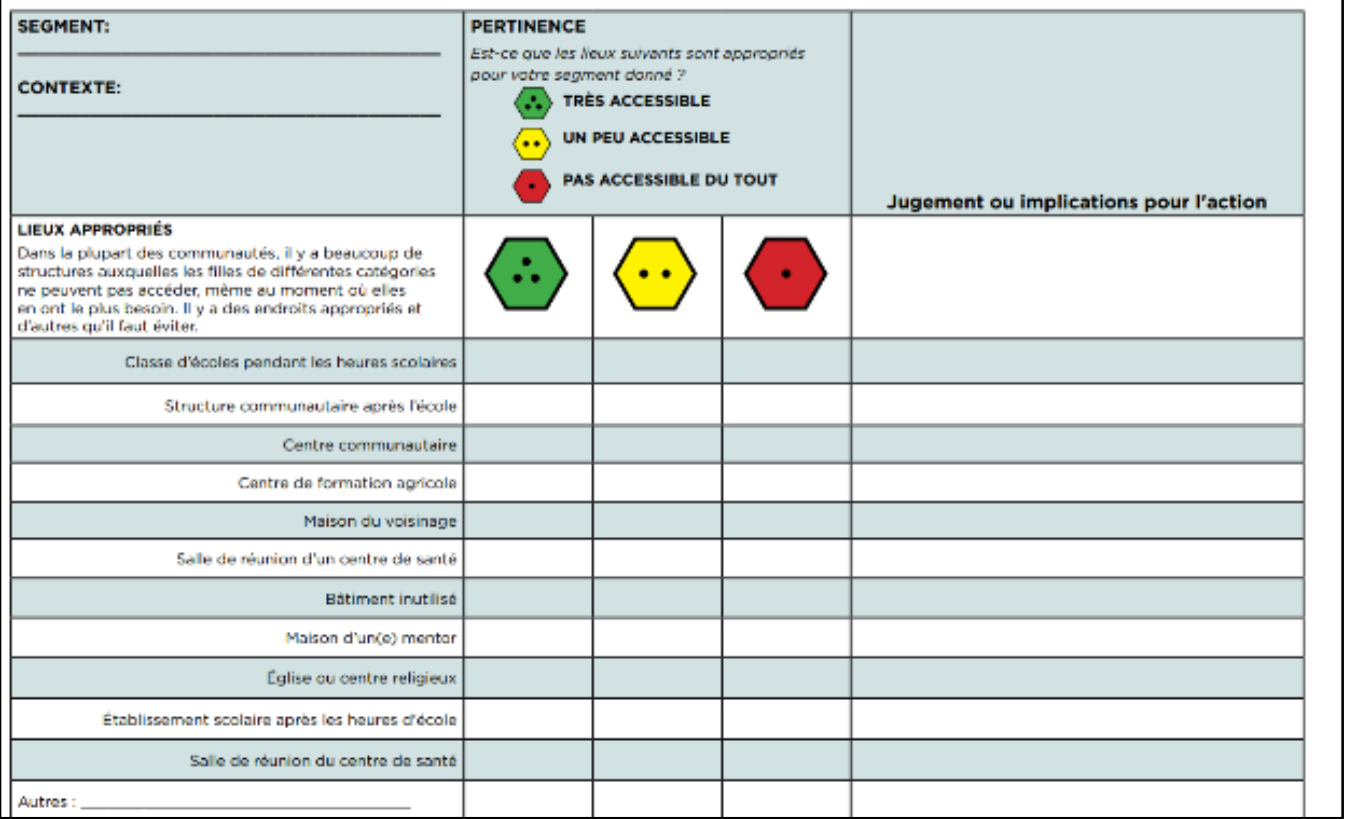

15 Adapté de : https://www.popcouncil.org/uploads/pdfs/2016PGY GirlsProtectiveAssetsTools fr.pdf (Pages 29- 31) 


\begin{tabular}{|c|}
\hline STRUCTURE DE LA SESSION DE L'ESPACE SÛR 16 \\
\hline La structure de l'espace sûr concerne : le lieu de rencontre, les jours de la semaine, les heures, \\
les adultes impliqués, etc. \\
\hline - Où trouver un lieu de rencontre sûr dans la communauté ? \\
- Où trouver un lieu de rencontre accessible dans la communauté ? \\
- À quelles heures les AJF peuvent-elles se rencontrer en sécurité ? \\
- Quels jours de la semaine les AJF peuvent-elles se rencontrer? \\
- Combien de fois par semaine et pendant combien de temps les AJF ont-elles le temps de se \\
- De quels adultes importants dans leur vie les AJF doivent-elles obtenir la permission de \\
- À qui les AJF s'adressent-elles et demandent-elles conseil et soutien en cas de difficulté ? \\
- De façon objective, quel est le coût d'opportunité pour l'AJF de répondre aux séances \\
retenues et comment le minimiser?
\end{tabular}

\section{Que signifient ces spécificités de la structure d'un espace sûr pour la conception de composants d'une manière centrée sur les AJF?}

Pour pouvoir créer un programme réussi, il faut adapter l'approche d'après les spécificités des AJF dans une communauté. II faut comprendre comment l'expérience qu'elles ont de leur environnement physique et social change avec la puberté.

En utilisant des informations spécifiques et détaillées (collectées à l'aide de certains outils énumérés ci-dessous) on peut identifier la population des AJF au sein d'une communauté. À savoir :

- Combien d'adolescentes y a-t-il ?

- Quelles sont leurs caractéristiques (scolarité, statut matrimonial, etc., vit avec les parents, les employées de maison, etc.) ?

- Qui est considéré comme " membre de la communauté "? (Les migrantes, les déplacées internes, les employées de maison, etc. sont-elles exclues des ressources et des opportunités de la communauté ?)

Cela vous permettra de visualiser la communauté du point de vue des AJF (Figure 4).

\footnotetext{
16 Adapté de "Conception d'un programme centré sur les AJF ; Boite à outils pour l'élaboration, le renforcement et l'expansion de programmes destiné aux adolescentes " du Population Council
} 


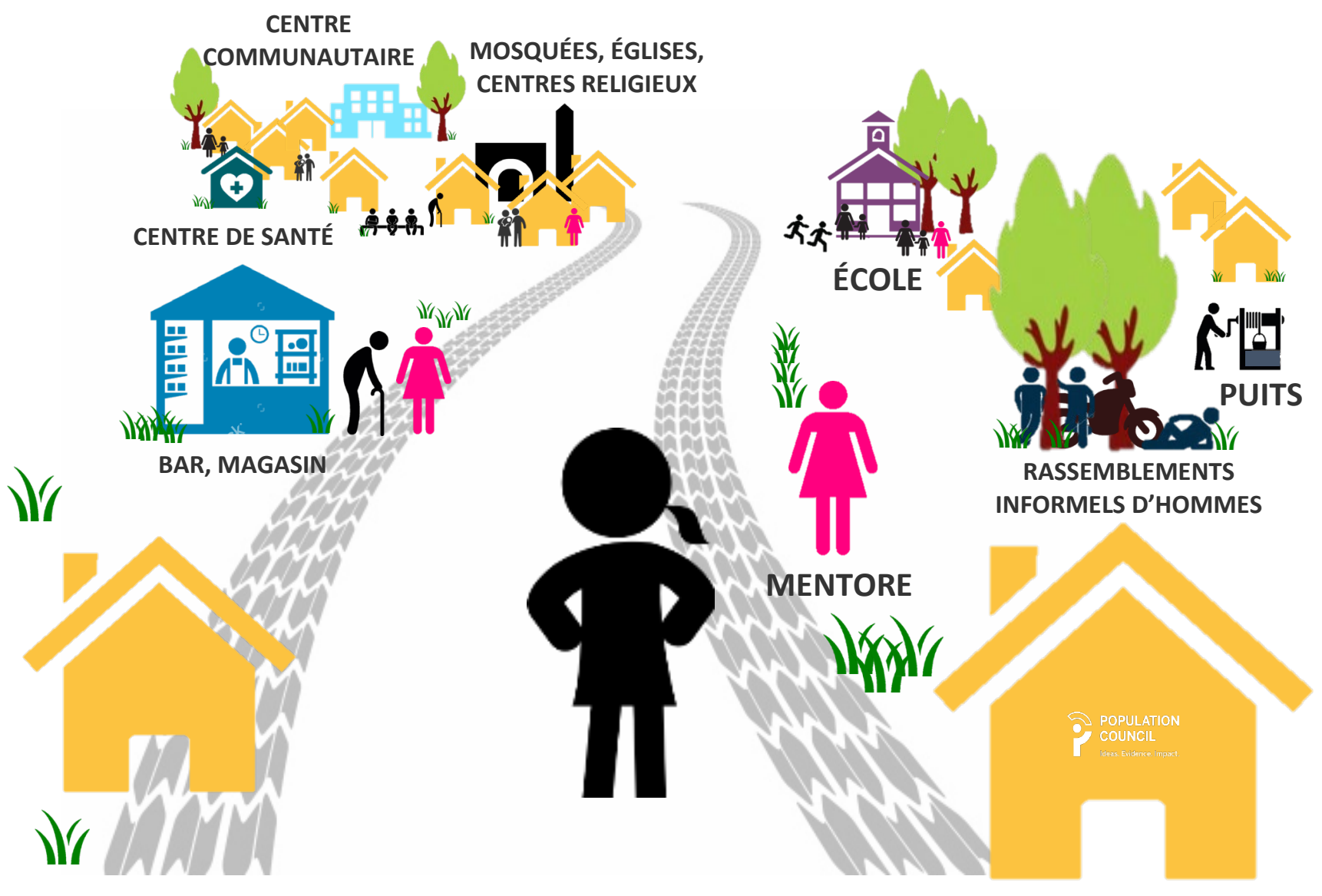

Certains outils et activités du Population Council peuvent vous fournir ces informations spécifiques sur les communautés dans lesquelles vous travaillerez avec les AJF :

- Le Girl Roster ; (décrit en détail plus haut dans ce document à la page 10)

- L'exercice de balayage communautaire ;

- L'exercice de cartographie communautaire et autres.

- Outils d'évaluation de la sécurité

Balayage des ressources communautaires 17

Le Balayage des Ressources Communautaires est un outil de programme qui fournit une description rapide des caractéristiques géographiques et sociales des communautés de AJF. Avec cet outil, le personnel du programme peut systématiquement explorer la zone d'intervention d'un programme en couvrant intentionnellement la région à pied tout en enregistrant les caractéristiques élémentaires et les structures clés, en utilisant un stylo et du papier ou une application de téléphone mobile. L'outil de programme est destiné à fournir un balayage de la communauté, et non un inventaire complet. Le Balayage de

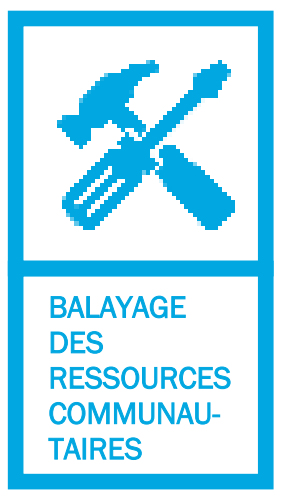

17 Adapté de https://www.popcouncil.org/uploads/pdfs/2016PGY GirlsProtectiveAssetsTools fr.pdf (Pages 16-17) 
Ressources Communautaires peut être utilisé en combinaison avec l'outil Le Girl Roster pour fournir une cartographie informative de l'endroit où les AJF habitent par rapport aux ressources environnantes.

L'intérêt du Balayage des Ressources Communautaires est de pouvoir éclairer les décisions prises par les chargé(e)s de programme pour savoir où situer les plateformes d'AJF, quelles ressources et structures existent dans la communauté, et comment mettre les AJF en contact avec les ressources dont elles ont besoin.

\section{Exercice de cartographie communautaire 18}

Les cartes communautaires sont des outils importants pour améliorer la compréhension de l'endroit où les AJF peuvent aller ou non dans la communauté. Cet outil aide les AJF à identifier les ressources communautaires clés ainsi que les zones qui peuvent être considérées comme problématiques ou dangereuses (écoles, marchés, sources d'eau, rassemblements d'hommes, bars, etc.). L'objectif est d'identifier les perceptions des AJF concernant les ressources et les structures communautaires, ainsi que les organisations ou les institutions importantes pour elles.

Il est utile de mettre en évidence les zones d'accès fréquent, occasionnel, rare et nul par les AJF, ainsi que les zones qui peuvent être dangereuses ou perçues

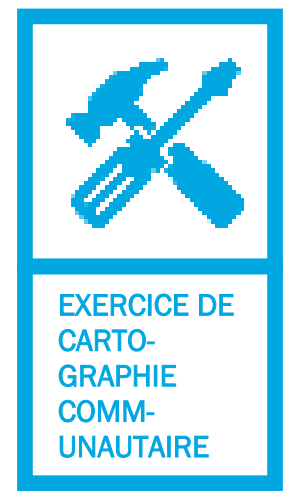
comme dangereuses, et les raisons de ces perceptions (Figure 5).

Figure 5 : Grille de la carte de caractéristiques du facilitateur

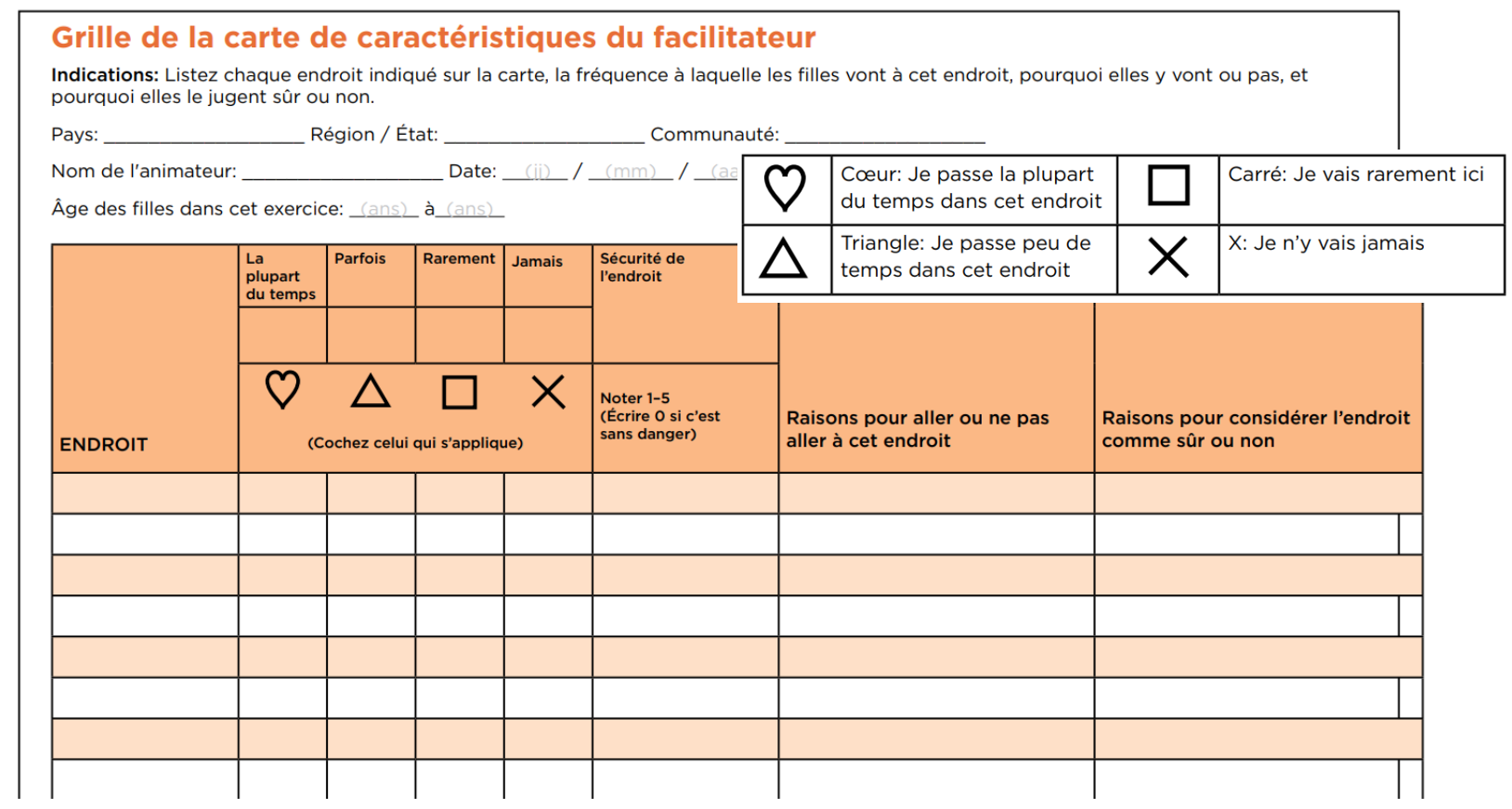

18 Adapté de https://www.popcouncil.org/uploads/pdfs/2016PGY GirlsProtectiveAssetsTools fr.pdf (Pages 19-21) 
Outils d'évaluation de la sécurité 19 :

Les outils d'évaluation de sécurité 20 aide le personnel de programme à comprendre les heures, les conditions et les situations dans lesquelles les AJF se sentent ou non en sécurité. Cet outil se compose des feuilles de travail (Figure 6) que les facilitateurs peuvent sélectionner pour explorer la sécurité dans des différents domaines, comme par exemple :

1. Moment de la journée,

2. Sécurité en fonction du jour de la semaine,

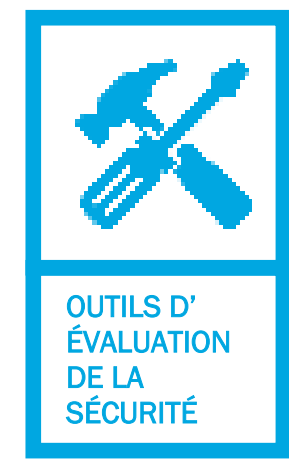

3. Période de l'année,

4. Différents endroits de la communauté,

5. Déplacements ou certaines activités (exemple ci-dessous),

6. Situation (confrontation avec un policier par exemple),

7. Réseaux sociaux (exemple ci-dessous).

19 Adapté de https://www.popcouncil.org/uploads/pdfs/2016PGY GirlsProtectiveAssetsTools fr.pdf Pages 26-28)

20 Austrian, K. and D. Ghati. 2010. Girl Centered Program Design: A Toolkit to Develop, Strengthen \& Expand Adolescent Girls Programs. Population Council. http://www.popcouncil.org/research/girl-centeredprogram-design-a-toolkit-to-develop-strengthenand-expand-ado 
Figure 6 : Exemple des outils d'évaluation de sécurité

\section{Sécurité lors des déplacements ou de certaines activités}

\begin{tabular}{|l|l|l|l|l|}
\hline Situation & Toujours en sécurite & $\begin{array}{l}\text { Parfois en } \\
\text { sécurite }\end{array}$ & $\begin{array}{l}\text { Jamais en } \\
\text { sécurite }\end{array}$ & Ce qui aiderait : \\
\hline $\begin{array}{l}\text { Prendre les transports } \\
\text { publics }\end{array}$ & & & & \\
\hline Prendre un taxi & & & & \\
\hline Services de santé & & & & \\
\hline Se rendre à lécole & & & & \\
\hline Se rendre à la banque & & & & \\
\hline Se rendre à la paste & & & & \\
\hline Sur un terrain de foot & & & & \\
\hline Au salon de beauté & & & & \\
\hline Á l'église/a la mosquée & & & & \\
\hline Autre & & & & \\
\hline
\end{tabular}

\section{Réseaux sociaux}

\begin{tabular}{|c|c|c|c|c|}
\hline Dans une situation d'urgence ... & Oui & Non & Pas sure & Remarques \\
\hline Avez-vous cinq amies en dehors de la famille? & & & & \\
\hline $\begin{array}{l}\text { Avez-vous un lieu ou retrouver vos amies au moins une } \\
\text { fois par semaine? }\end{array}$ & & & & \\
\hline $\begin{array}{l}\text { Avez-vous une personne vers laquelle vous tourner } \\
\text { en cas de problème personnel (problème vous } \\
\text { concernant ou concernant une autre personne)? }\end{array}$ & & & & \\
\hline $\begin{array}{l}\text { Connaissez-vous une personne à qui vous pouvez } \\
\text { emprunter de l'argent? }\end{array}$ & & & & \\
\hline Avez-vous un lieu sûr oú posser la nuit? & & & & \\
\hline $\begin{array}{l}\text { Avez-vous une personne vers laquelle vous tourner en } \\
\text { cas de problème de santé? }\end{array}$ & & & & \\
\hline $\begin{array}{l}\text { Avez-vous une personne vers laquelle vous tourner en } \\
\text { cas de probleme économique? }\end{array}$ & & & & \\
\hline
\end{tabular}

Toutes ces spécificités locales sont importantes pour une conception intentionnelle des programmes centrés sur les AJF comme les espaces sûrs de SWEDD et peuvent améliorer la sécurité des AJF dans leurs communautés (e.g. la fourniture de bons de transport, la promotion de l'installation de lumières, etc.). 
Afin de mettre en œuvre des projets de transformation en matière de genre, comme les espaces sûrs de SWEDD, il est essentiel d'obtenir le soutien des chefs de communauté, des parents et des autres membres de la communauté. Ces acteurs sont essentiels au succès de tout programme d'espace sûr, car ils sont les gardiens des ressources de la communauté ainsi que les participants eux-mêmes.

En savoir plus sur les attitudes et les croyances de la communauté a des implications pratiques en termes de réduction de l'opposition à votre programme et des réactions négatives potentielles.

Actuellement, tous les pays SWEDD s'engagent avec les parties prenantes de la communauté. Une approche est en cours de mise en œuvre dans un pays SWEDD qui s'est avérée prometteuse pour l'engagement communautaire. Il s'agit de la création d'une "Session Zéro " dans le programme de mentorat. Dans ce pays, l'équipe a conçu la nouvelle session pour guider les mentores dans la présentation du concept et du contenu des espaces sûrs aux membres de la communauté et aux dirigeants locaux. Cette stratégie s'est avérée fructueuse et a permis de créer une relation de confiance entre le personnel et les activités du projet, et les communautés où les espaces sûrs étaient mis en œuvre. Dans le même ordre d'idées, un autre pays a revu ligne par ligne le curriculum d'études avec les dirigeants locaux afin d'être transparent et direct avec la communauté sur le contenu du programme des espaces sûrs. Ces approches pourraient contribuer à dissiper toute préoccupation potentielle concernant les leçons incluses dans les espaces sûrs.

\section{DES NORMES MINIMALES POUR UNE EXPANSION DE QUALITÉ DES ESPACES SÛRS}

Ce qui fonctionne dans les petits projets n'est pas le même que ce qui va fonctionner à grande échelle, l'expansion doit être planifiée tout en maintenant la qualité qu'une surveillance étroite peut aider. Pour une mise en échelle avec qualité, une prise de décision intentionnelle est essentielle pour concevoir le où, le qui, le quoi et le comment.

Une approche visant à optimiser la couverture consiste à utiliser les données existantes pour localiser les points chauds, ce qui peut aider à identifier de nouvelles communautés et populations de zones de chalandise. L'outil Girl Roster (décrit à la page 10) a été utilisé afin de rendre les communautés visibles et recueillir les informations nécessaires pour aider à façonner les programmes et à établir des priorités pour les groupes cibles.

Ces informations vous aideront à décider où étendre la mise en œuvre d'espaces sûrs. II faut utiliser des informations au niveau de la communauté pour identifier et investir dans les " épicentres " où le besoin est plus élevé (c'est-à-dire : un taux élevé de mariage précoce, taux de fécondité élevé, taux de scolarisation bas chez les AJF, etc.).

Après avoir identifié ces zones "épicentres " d'après des informations actuelles au niveau de la communauté, vous seriez en position de simplifier le recrutement des AJF puisque la communauté entière est plus à risque donc on pourrait en principe recruter toutes les AJF dans cette communauté qui bénéficieront des espaces sûrs dans cette communauté. 
De même, la superposition/le chevauchement d'autres activités de la sous-composante $1.2^{21}$ et aussi d'autres composantes du SWEDD dans ces "épicentres" de risque aux AJF apportera les mêmes bénéfices que la superposition à petite échelle comme décrit dans la section " $\underline{A m p l i f i e r}$ les avantages du SWEDD en superposant les activités du SWEDD et en coordonnant les contenus" (Étape 2, Page 57)

Quant au curriculum, les variations de contenu seront encore plus importantes pour un programme à grande échelle. Un ensemble de programmes de base en plus des adaptations guidées spécifiques au segment seraient nécessaires pour répondre aux besoins de chacun.

Combien cela va-t-il coûter? Veiller à ce que les plans du programme d'espace sûr soient $\underline{\text { budgétisés }}$

Les éléments à budgétiser doivent comprendre :

- Allocation de mentore ;

- Location, rénovation d'espaces ;

- Fournitures pour les AJF ;

- Copies de matériel d'apprentissage ;

- Activités de recrutement ;

- Activités d'introduction dans la communauté ;

- Salaires du personnel ;

- Transport ; et

- Téléphone et crédit/unité pour la communication.

Il existe des éléments nécessaires qui doivent être payés pour que le projet soit couronné de succès. Les considérations pour certains éléments à budgétiser au niveau d'un espace sûr sont présentées dans le Tableau 2.

21 La sous-composante 1.2 contribuera à l'amélioration de l'accès aux informations et services relatifs à la santé sexuelle et reproductive, à l'augmentation des compétences de vie, au maintien des filles à l'école ainsi qu'à l'autonomisation économique des filles non scolarisées, déscolarisées et des filles sans emploi. 


\begin{tabular}{|c|c|}
\hline $\begin{array}{l}\text { Allocation de Mentore/ } \\
\text { de superviseur } \\
\text { dech }\end{array}$ & $\begin{array}{l}\text { La rémunération doit être régulière, à temps et suffisante pour le travail } \\
\text { effectué par le superviseur ou la mentore. }\end{array}$ \\
\hline & $\begin{array}{l}\text { Dans l'idéal, les communautés feront don d'un espace sans frais. Dans } \\
\text { certains cas, un budget peut être nécessaire pour la location de l'espace. } \\
\text { Dans tous les cas, des fournitures et des décorations pour l'espace sont } \\
\text { nécessaires. Il s'agira probablement de peinture, de tapis, de bancs, } \\
\text { d'autres meubles, de jeux, de décorations, de fournitures artistiques, d'un } \\
\text { coffre-fort (pour stocker le matériel lorsqu'il n'est pas utilisé) }\end{array}$ \\
\hline s pour les AJF & $\begin{array}{l}\text { Il faut décider ce qu'il faut acheter au minimum pour la participation et la } \\
\text { rétention des AJF. } \\
\text { Des éléments qui sont le plus essentiels seront des collations, de l'eau } \\
\text { potable et des matériaux de bureau (cahiers et crayons). On peut } \\
\text { également leur fournir des produits hygiéniques féminins réutilisables et } \\
\text { d'autres articles utiles. } \\
\text { Mais vous devez considérer comment les coûts supplémentaires peuvent } \\
\text { affecter votre capacité à atteindre les AJF à l'échelle et viser à fournir le } \\
\text { minimum de ce dont chaque fille a besoin pour s'engager pleinement dans } \\
\text { le programme. }\end{array}$ \\
\hline $\begin{array}{l}\text { Copies } \\
\text { d'appre } \\
\text { d'autres }\end{array}$ & $\begin{array}{l}\text { Il s'agit généralement d'un matériel imprimé, tableau de papier, feutres } \\
\text { etc. } \\
\text { Exemplaires des formulaires pour le suivi, évaluation et apprentissage au } \\
\text { niveau de l'espace sûr et aussi au niveau de la supervision (peut être une } \\
\text { tablette). } \\
\text { D'autres fournitures seront probablement des listes de présence/des } \\
\text { registres et beaucoup de stylos! }\end{array}$ \\
\hline & $\begin{array}{l}\text { Les coûts associés avec le transport pour les superviseurs et les mentores } \\
\text { devraient être pris en compte. Ces mesures n'affecteront pas toutes les } \\
\text { mentores ou tous les superviseurs de la même manière. } \\
\text { N'oubliez pas que l'accès à l'espace sûr est essentiel (pour la mise en } \\
\text { œuvre et le suivi) et que le coût du transport est un élément important } \\
\text { pour rendre un lieu accessible ou non. }\end{array}$ \\
\hline $\begin{array}{l}\text { Téléphone et crédit/unité } \\
\text { pour la communication }\end{array}$ & $\begin{array}{l}\text { Des deux côtés, les mentores et les superviseurs doivent pouvoir se } \\
\text { contacter. La fourniture d'un crédit téléphonique mensuel peut faciliter le } \\
\text { soutien que le superviseur peut apporter à la mentore et que les mentores } \\
\text { peuvent se donner mutuellement. }\end{array}$ \\
\hline $\begin{array}{l}\text { Activités d'introduction } \\
\text { dans la communauté }\end{array}$ & $\begin{array}{l}\text { Activités d'introduction et de recrutement dans les communautés et avec } \\
\text { les parents (dialogues communautaires). Ces activités pourraient inclure } \\
\text { des rafraîchissements, du crédit téléphonique pour le personnel et peut- } \\
\text { être la location d'une salle. }\end{array}$ \\
\hline $\begin{array}{l}\text { Formation/recyclage des } \\
\text { mentores }\end{array}$ & $\begin{array}{l}\text { Les mentores et les superviseurs auront besoin d'une formation initiale } \\
\text { dans tous les domaines d'interventions ainsi que d'une formation de } \\
\text { recyclage et de possibilités de mise en réseau avec d'autres mentores. Ces } \\
\text { coûts comprennent les déplacements, les indemnités journalières de } \\
\text { formation, le lieu de la formation, etc. }\end{array}$ \\
\hline
\end{tabular}




\section{ÉTAPE 2: MISE EN CEUVRE DU PROGRAMME}




\section{ÉTAPE 2 : MISE EN OEUVRE DU PROGRAMME}

Cette section se concentre sur la manière dont les espaces sûrs du SWEDD peuvent être mis en œuvre avec succès. Les lecteurs découvriront des moyens utiles de recruter des mentores et AJF appropriées, de transmettre des contenus pertinents aux AJF ainsi que des moyens de mettre les AJF en contact avec les ressources communautaires existantes. Cette partie va traiter les sujets suivants :

- Les ressources humaines,

- Le contenu des espaces sûrs,

- Les sessions d'espaces sûrs engageantes et dynamiques,

- Les liens et soutiens pour l'utilisation d'autres ressources communautaires, et

- Les stratégies pour motiver et maintenir une fréquentation régulière des AJF.

\section{DES NORMES MINIMALES POUR DES RESSOURCES HUMAINES ADÉQUATES}

\section{Un personnel formé pour assurer une planification stratégique efficace, une mise en œuvre harmonieuse et un SEA informatif}

Ce n'est pas seulement dans les espaces sûrs que la mise en œuvre d'une programmation de qualité nécessite de prévoir des ressources humaines adéquates, des financements et des mécanismes de mise en œuvre réalistes. Cela peut constituer un défi particulier lors de la planification de programmes communautaires qui se déroulent dans des zones rurales à faibles ressources comme dans le cadre du SWEDD, souvent hors de portée des systèmes sociaux et des infrastructures publiques existants. II est essentiel d'élaborer délibérément des lignes directrices en matière de ressources humaines, basées sur une analyse réaliste du nombre de personnes et de leurs compétences - nécessaires à chaque niveau de programmation. Cela inclut les ressources humaines au niveau national, au niveau de la coordination (UGP), au niveau de la mise en œuvre (ONG et/ou ministères), et au niveau de la supervision et du mentorat.

Le personnel du partenaire de mise en œuvre devrait avoir :

- Une capacité de planification stratégique, de prise de décision, de sensibilité au genre,

- Des connaissances sur le SEA,

- Un leadership, y compris le leadership en cascade, et

- Un plan réaliste des ressources humaines (et suffisamment de superviseurs - qui doivent également être formés, soutenus, et assistés). 


\section{Un cadre de mentores adéquatement équipées, soutenues et habilitées}

En tant que principales transmetteuses du contenu du programme, les mentores jouent un rôle déterminant quant au succès des espaces sûrs. Elles doivent également être considérées comme un groupe bénéficiaire du programme. Pour effectuer leur travail efficacement et être retenues, elles ont besoin de plusieurs éléments qui sont énumérés dans le Tableau 3 ci-dessous. ${ }^{22}$

Tableau 3: Les composantes essentielles d'un cadre de mentores adéquatement équipées, soutenues et habilitées

\begin{tabular}{|l|l|} 
Critères d'éligibilité des \\
mentores des espaces sûrs \\
du SWEDD
\end{tabular}

22 Adapté de "Valoriser le potentiel des mentores: Recrutement, formation, et soutien des mentores pour des programmes dédiés aux adolescentes" du Population Council https://www.popcouncil.org/uploads/pdfs/2019PGY_MentorToolkit_fr.pdf 
Quelles sont les qualités d'une bonne mentore ? Choisir les bonnes personnes est la clé d'une mise en œuvre réussie du programme. L'expérience montre qu'une caractéristique essentielle des mentores est leur origine et leur lieu de résidence ; qu'elles soient locales, qu'elles viennent de la communauté qu'elles servent et qu'elles y vivent. Pourquoi est-ce important?

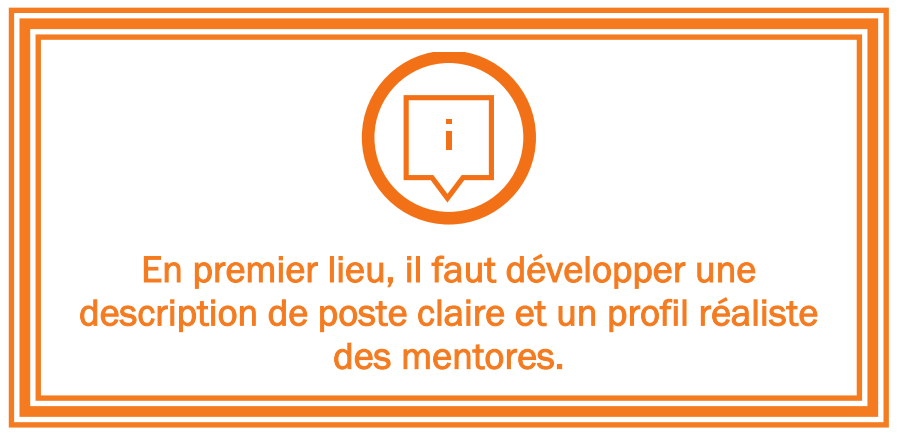

- Le fait d'être locale devrait accroître la capacité des mentores à comprendre et à répondre aux défis auxquels les AJF sont confrontées et permettre aux AJF de s'identifier à elles.

- Le fait d'être de la communauté augmente les probabilités que les parents et les tuteurs fassent confiance aux mentores et permettent aux AJF de participer. Cela garantit aussi l'accessibilité des mentores en cas de besoin en dehors des heures de réunion habituelles.

- Le recrutement local est un investissement dans le leadership féminin, qui permet aux AJF plus âgées et aux jeunes femmes ayant un potentiel de leadership de développer et d'exercer des compétences qui seraient autrement négligées.

- Cela peut remettre en question les normes communautaires sur l'accès des AJF et des jeunes femmes aux ressources, en fournissant des modèles alternatifs positifs pour les AJF dans leur communauté et en leur donnant plus de "voix" dans la prise de décision dans leur espace de vie.

II ne faut pas oublier que ce qui est idéal n'est pas toujours réaliste. II faudrait s'enrichir des expériences de la mise en œuvre des espaces sûrs pendant SWEDD I et rassembler les attributs minimums pour une mentore appropriée. Quelles adaptations sont nécessaires pour SWEDD II afin de faire face aux différents défis qui se présenteront?

La suite est un exemple de liste de qualifications qui peuvent être utilisées comme critères de sélection des mentores.

La liste précise également les conditions requises pour être une enseignante ou une mentore communautaire, dont les rôles diffèrent dans le programme à l'origine de cet outil. L'outil énumère également les étapes de sélection des mentores.

\section{Cette liste doit être adaptée afin d'inclure des critères appropriés et pertinents pour chaque projet et contexte}

Une mentore est une femme âgée de 20 à 40 ans résidante dans la même communauté que l'espace sûr (ou à proximité : régulièrement accessible et sûr) et ayant terminé un certain niveau de scolarisation (qui est logique pour le contexte). Elle doit également avoir certaines qualifications/exigences :

- Alphabète/peut lire et écrire ;

- Respectée dans la communauté, servant de modèle aux jeunes femmes ;

- Posséder des qualités de leader ;

- Expérience de travail avec la communauté et les groupes vulnérables ;

- Expérience en matière de formation et/ou d'animation de groupes ; 
- Connaissances de base en matière de santé sexuelle et reproductive préférées ;

- La volonté et la flexibilité de répondre aux préoccupations des AJF en dehors des heures d'ouverture de l'espace sûr ;

- Engagée à améliorer la situation des AJF dans sa communauté ;

- L'expérience de l'utilisation de la technologie telle que les téléphones intelligents ou les tablettes constitue un avantage supplémentaire ;

Étapes de la sélection des mentores 23

- Liste restreinte de mentores : trois personnes par catégorie

- Établir un calendrier d'entretiens avec le partenaire local

- Appel aux candidats pour les entretiens

- Mener les entretiens par un panel de l'école (si elle est basée à l'école) ou de la communauté (si elle est basée dans la communauté) : 2-3 membres et le partenaire local

- Sélectionner un candidat

- Informer le partenaire local concerné

- Invitation à la formation (la lettre sera fournie par un partenaire local)

II est parfois difficile dans les pays SWEDD d'identifier des candidates locales qui ont le niveau d'éducation et d'alphabétisation désiré ou idéal pour suivre un curriculum et ensuite l'apprendre aux AJF. II existe d'autres caractéristiques importantes des mentores à sélectionner quand le besoin se présente et il y a des expériences et des matériaux qui peuvent être utilisés comme décrit dans l'étude de cas AGI-K ci-dessous et adaptés à un contexte différent.

${ }^{23}$ Adapté de "Valoriser le potentiel des mentores: Recrutement, formation, et soutien des mentores pour des programmes dédiés aux adolescentes" du Population Council https://www.popcouncil.org/uploads/pdfs/2019PGY_MentorToolkit_fr.pdf 


\section{ÉTUDE DE CAS - INITIATIVE ADOLESCENT GIRLS-KENYA : PROGRAMME ADAPTATION POUR LES MENTORES FAIBLEMENT ALPHABÉTISÉES}

Le Population Council a mis en œuvre un programme, "the Adolescent Girls InitiativeKenya (AGI-K), qui a testé des combinaisons d'interventions en matière de santé, de prévention de la violence, de création de richesse et d'éducation afin de déterminer quel ensemble d'interventions a le plus d'impact et à quel coût. Ce programme a été mis en œuvre à Wajir, une région isolée et sous-développée du Kenya, le long de la frontière nordest avec la Somalie.

En raison des faibles taux d'alphabétisation et des différences d'expérience en matière de facilitation dans le site AGI-K de Wajir, le Population Council et ses partenaires d'exécution étaient en présence de difficultés à recruter et à retenir des mentores capables de faciliter le curriculum de l'espace sûr. Environ six mois après le lancement, l'équipe du programme a reconnu qu'elle devait adapter le programme pour que Wajir puisse s'adresser à des mentores peu alphabétisées.

Les membres de l'équipe ont traduit les curricula écrits en scripts audio avant de les traduire dans la langue locale. Ils ont par la suite fait des enregistrements audios de chaque session afin de les copier sur des clés USB, et remis à chaque mentore une simple radio avec un port USB. Le coût de chaque radio et de chaque USB était approximativement de 30 dollars par mentore.

Les sessions audios ont aidé les mentores à animer efficacement les séances, même si leur niveau d'alphabétisation était faible. Au-delà de la question de l'alphabétisation, les sessions audios ont permis d'ouvrir le dialogue entre les mentores et les participants sur les questions de santé sexuelle et reproductive souvent évitées dans la communauté plus réservée de Wajir. De plus, les sessions audios ont permis de standardiser leur contenu, car l'information était fixée sur l'enregistrement et non sur le mentor individuel qui décidait de ce qu'il fallait dire ou ne pas dire lors de l'animation des sessions.

(Adapté de "Valoriser le potentiel des mentores: Recrutement, formation, et soutien des mentores pour des programmes dédiés aux adolescentes" du Population Council)

Pour plus d'informations: Austrian, K., E. Soler-Hampejsek, J. Mumah, B. Kangwana, Y. Wado, B. Abuya, V. Shah, and J. Maluccio. 2018. “Adolescent Girls Initiative-Kenya: Midline Results Report." Nairobi: Population Council.

\section{Rémunération équitable}

Les mentores ne sont pas des bénévoles; leur travail est d'une grande importance et doit être rémunéré de manière appropriée. En les rémunérant, on augmente les chances qu'elles soient dévouées au programme et qu'elles fournissent un travail de qualité. Cela permet également de démontrer que le travail qu'elles accomplissent est précieux et crée une opportunité de subsistance pour les jeunes femmes de la communauté. À l'inverse, ne pas les payer décemment peut entraîner une rotation sur une distance plus importante et augmenter les coûts de formation, réduisant ainsi la durabilité du programme. Ne pas les payer suggère que leur travail n'est pas précieux ni valorisé. 
Les salaires varient d'un programme à l'autre en fonction du lieu et des attentes des mentores (en termes de temps consacré et de tâches). Toutefois, les programmes devraient toujours verser aux mentores un montant similaire aux salaires pratiqués dans la communauté pour un travail comparable, comme les agents du gouvernement ou les agents

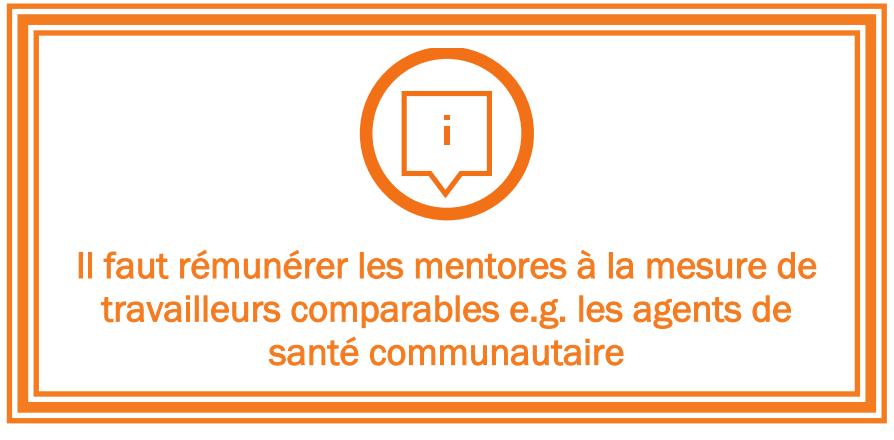
de santé communautaires. Dans certains contextes, les mentores ont reçu des primes pour diriger des groupes supplémentaires, et non pour remplir et soumettre des informations sur l'assiduité ou d'autres outils de suivi, car ces tâches font partie des attentes pour le rôle de mentor. Certains programmes offrent une rémunération supplémentaire, comme des vélos, le paiement du transport, le déjeuner, des bottes de pluie et des imperméables, des primes de vacances, des crédits de téléphone et des forfaits Internet pour les téléphones, et des augmentations de salaire pour chaque année passée en tant que mentore (par exemple, une augmentation de 5 à $10 \%$ ).

Le paiement lié à l'accomplissement des responsabilités énoncées dans le contrat de la mentore est important. Toutefois, les contrats détaillés peuvent également susciter des plaintes de la part des mentores lorsqu'il leur est demandé d'effectuer des tâches qui ne relèvent pas de leurs responsabilités habituelles. II sert de s'entendre à l'avance avec les mentores sur les attentes claires quant à leurs responsabilités tout en ne limitant pas trop les petites tâches supplémentaires qui peuvent les aider à accomplir leur travail sans exiger une rémunération plus élevée. Quoique difficile à appliquer dans la pratique, certains programmes prévoient des dispositions visant à réduire la rémunération des mentores dans le cas où elles manquent des séances ou falsifient des dossiers. Les mentores apprécient également les avantages non financiers, tels que les certificats délivrés à l'issue de la formation, l'expérience du mentorat, le développement de nouvelles compétences et la reconnaissance ou les récompenses pour un engagement ou des performances exceptionnels. Par exemple, le programme Abriendo Oportunidades au Guatemala offre aux mentores la possibilité de participer à une formation spéciale sur des sujets d'intérêt, tels que les droits de l'homme et la recherche sociale. Le statut social de la mentore au sein de la communauté peut également constituer une motivation pour les mentores.

Les modes de rémunération des mentores prennent diverses formes. La plupart des programmes exigent des mentores qu'elles ouvrent un compte bancaire - le premier de ce genre pour la plupart d'entre elles - et qu'elles déposent leurs allocations mensuelles après avoir remis leur rapport mensuel. Les programmes peuvent également envisager des transferts d'argent par téléphone portable pour payer les mentores lorsqu'elles sont disponibles. Les paiements en espèces sont déconseillés car ils peuvent exposer les mentores à des risques de vol. 24

\footnotetext{
${ }^{24}$ Adapté de "Valoriser le potentiel des mentores: Recrutement, formation, et soutien des mentores pour des programmes dédiés aux adolescentes" du Population Council https://www.popcouncil.org/uploads/pdfs/2019PGY_MentorToolkit_fr.pdf
} 


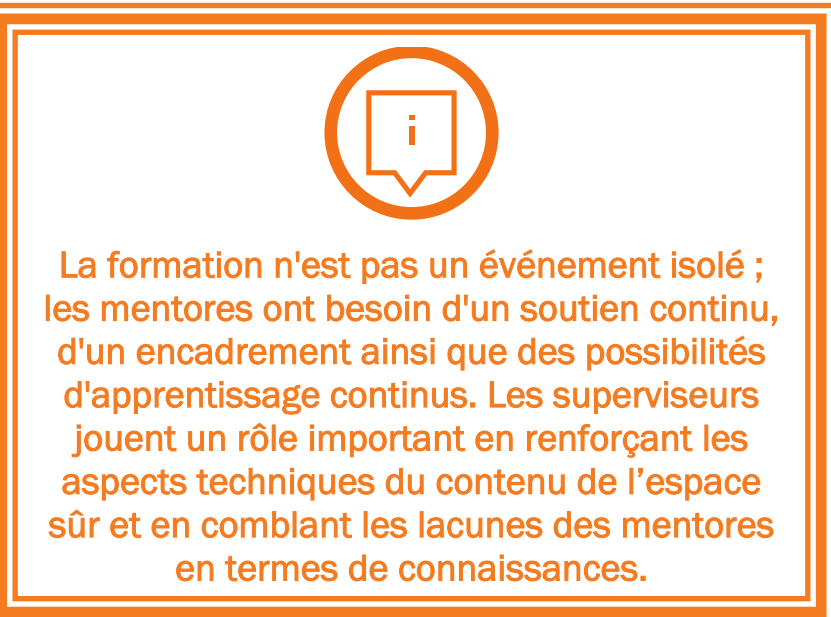

Pour que le programme soit couronné de succès, il est essentiel que les mentores soient formées et recyclées de manière adéquate pour remplir leur rôle. Les mentores reçoivent une formation sur les curriculums qu'elles vont dispenser, les comportements à tenir en tant que mentore, ainsi que leurs responsabilités en matière de suivi. Elles doivent être à l'aise avec le matériel du programme et pratiquer de nouvelles techniques d'enseignement et d'animation. De nombreuses personnes en formation sont censées enseigner un matériel qui ne leur est pas familier ; leur formation est l'occasion de transmettre des valeurs clés, en particulier autour de sujets sensibles comme la santé sexuelle et reproductive. Lorsque vous organisez une formation initiale de mentores, vous devez vous assurer que la formation aborde les multiples aspects du mentorat. Cette formation peut s'étendre sur une période de quatre jours à deux semaines et comprend généralement le curriculum à dispenser, des sujets tels que les compétences de gestion de groupe, la discussion des responsabilités de la mentore, et aborde aussi des sujets comme les visites à domicile ou les réunions

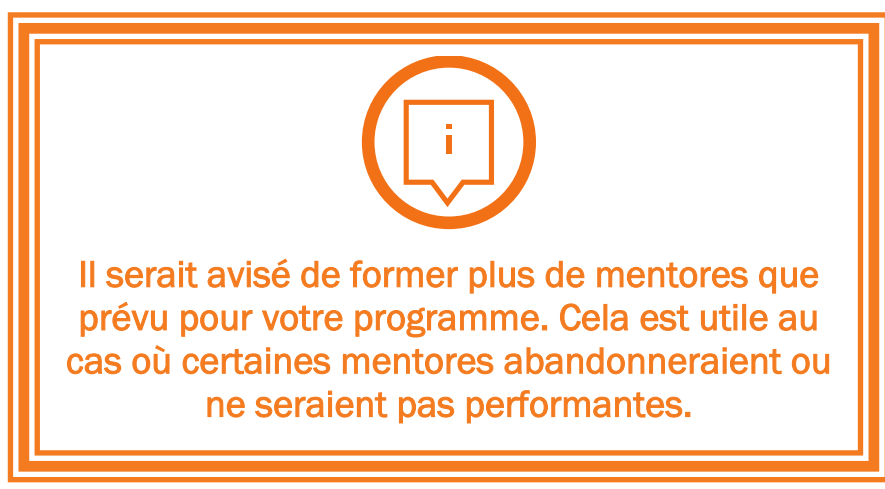
communautaires.

Lorsqu'il est requis des mentores d'utiliser des téléphones portables ou des tablettes, les programmes devraient également inclure des formations sur ces compétences particulières.

Les formations doivent être interactives et offrir aux mentores la possibilité de jouer des rôles et de mettre en pratique les compétences qu'elles utiliseront dans le cadre du programme.

La durée appropriée de la formation initiale sera déterminée par la quantité et le type de matériel que les mentores sont censés maîtriser, et par le niveau de familiarité des mentores avec le contenu et les approches.

Les opportunités pour les mentores d'apprendre et de pratiquer leurs compétences suivent souvent une formation initiale. Ces opportunités ont généralement lieu lors de formations de mise à jour organisées deux à trois mois après le début d'un programme, et lors de réunions hebdomadaires ou mensuelles avec les coordonnateurs de site. Au cours de ces réunions 
mensuelles ou hebdomadaires, les mentores soumettent leurs données de présence, discutent des succès et des défis, et suivent de brèves formations en rapport avec les sessions à venir. 25

\section{Formation sur le genre, les VBG et orientation vers les services appropriés}

La formation sur le genre, les VBG et orientation vers les services appropriés, doit aider les mentores à comprendre et à décrire les concepts clés et les questions fondamentales qui soustendent toutes les formes de VBG, ainsi qu'aider à :

- Accroître la capacité des mentores à discuter des concepts clés de manière à ce qu'elles puissent être bien comprises par la communauté et les AJF dans les espaces sûrs ;

- Identifier la relation entre l'abus de pouvoir et les VBG ;

- Comprendre que le terme "violence" dans le contexte des VBG signifie l'utilisation d'un certain type de force, qui peut ou non inclure la force physique ;

- Comprendre la signification du "consentement éclairé" et son lien avec les VBG ;

- Comprendre la relation entre les droits de l'homme et les VBG. ${ }^{26}$

Ainsi, pendant cette formation, vous voulez vous assurer que les mentores comprennent et sont capables de définir et de différencier : les VBG de l'exploitation sexuelle ; les violences sexuelles des VBG, les contacts physiques qui sont acceptables ou non, les manières de toucher qui sont déroutantes. Ce guide explorera plus profondément comment la mentore peut aider une AJF victime de VBG dans la section "Un système de référence solide et des canaux de référence pour les VBG dans la communauté " (Étape 2, Page 55) mais il est important qu'elle comprenne les VBG pour mieux aider les AJF.

Les mentores doivent également être capables d'enseigner et d'aider les AJF à comprendre et surtout enseigner dans les espaces sûrs : comment se sortir d'une situation d'exploitation ; comment signaler la violence ; comment prévenir et traiter les abus sexuels ; prévenir un viol, les abus sexuels et les scénarios de violence familiale ; comment traiter les abus sexuels et les viols ; prévenir les avances non désirées, etc.

De plus, vous voudriez que les mentores soient habilitées à connaître les termes suivants :

Consentement : Donner la permission ou l'accord pour que quelque chose se produise

Viol par un petit ami : viol entre des personnes qui se fréquentent ou se connaissent ; également appelé viol par une connaissance

\footnotetext{
25 Adapté de "Valoriser le potentiel des mentores: Recrutement, formation, et soutien des mentores pour des programmes dédiés aux adolescentes" du Population Council https://www.popcouncil.org/uploads/pdfs/2019PGY MentorToolkit fr.pdf

${ }^{26}$ Adapté de "Valoriser le potentiel des mentores: Recrutement, formation, et soutien des mentores pour des programmes dédiés aux adolescentes" du Population Council https://www.popcouncil.org/uploads/pdfs/2019PGY_MentorToolkit_fr.pdf
} 
Atteinte/Exploitation sexuelle sur mineur : Relations sexuelles avec une personne que la loi définit comme trop jeune pour consentir légalement, qu'elle ait consenti ou non (AJF de moins de 18 ans)

Exploitation : Utiliser une situation à son propre avantage sans se soucier de ce que ressent l'autre personne ; parfois en utilisant la force, la pression ou des ruses

Le genre : Ce qu'une société ou une culture attend de vous selon que vous êtes un homme ou une femme (rôles, comportements, etc.)

Les violences basées sur le genre (VBG) : Tout acte qui entraîne ou susceptible d'entraîner un préjudice physique, sexuel ou psychologique ou la souffrance d'une personne (garçon ou fille) sur la base des attentes et des suppositions concernant le rôle des sexes 27

II convient de renforcer les capacités des mentores afin qu'elles puissent fournir aux bénéficiaires d'un espace sûr une liste complète des ressources communautaires locales et leurs coordonnées. Ces ressources comprennent les services de de signalement des VBG, les services de conseil psychosocial pour les survivantes de violences, les services juridiques (il peut être utile de faire des fiches avec des images des ressources communautaires), ainsi qu'une liste des organisations de la communauté dédiées aux violences ou abus sexuels (si telles organisations existent dans la communauté ou les environs). 28

En résumé, les mentores doivent être formées à entreprendre des activités de prévention sur la violence et à apporter aux victimes de viols, souvent très jeunes, une information et un soutien adapté à leur culture. II est donc important, lors de la formation des mentores, de couvrir les concepts clés et les questions fondamentales qui sous-tendent les VBG. ${ }^{29}$

Un outil de formation des mentores sur les VBG se trouvent dans le document: "Valoriser le potentiel des mentores".

\section{Soutien social avec d'autres mentores
et soutien technique adéquat des superviseurs}

Les mentores peuvent s'aider mutuellement à s'épanouir lors de rencontres programmées sur une base régulière dans un espace précis où elles peuvent partager leurs défis et leurs succès. Par exemple, en donnant aux mentores l'occasion de se réunir et de s'exercer à animer des séances entre elles, on renforce le capital social et on les aide à se motiver, à apprendre les unes des autres et à se soutenir mutuellement. Cela les aide également à construire leurs réseaux sociaux.

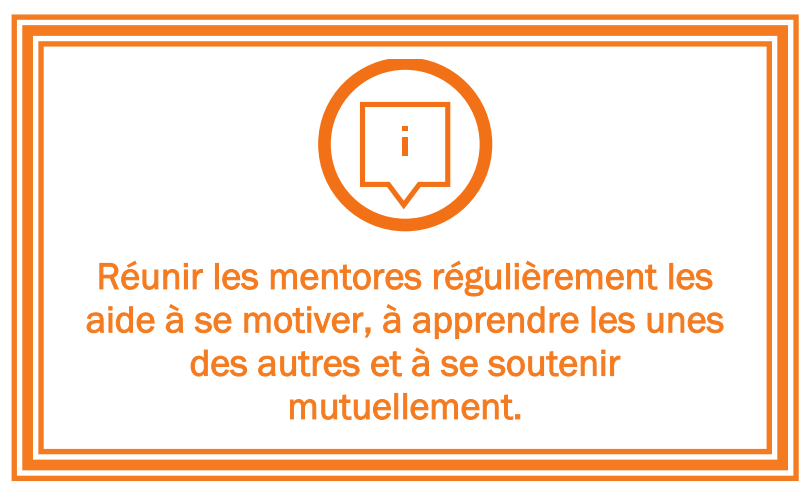

27 Adapté de " AGI-K Health Life Curriculum Wajir " du Population Council

28 Adapté de "AGI-K Health Life Curriculum Wajir " du Population Council

29 Adapté de "Valoriser le potentiel des mentores: Recrutement, formation, et soutien des mentores pour des programmes dédiés aux adolescentes" du Population Council https://www.popcouncil.org/uploads/pdfs/2019PGY_MentorToolkit_fr.pdf 
Après une formation initiale, il est utile de réunir les mentores régulièrement, par exemple tous les mois, pour discuter entre elles (et avec un superviseur) de ce qui se produit dans leur groupe et de toutes les zones floues sur lesquelles elles pourraient avoir besoin d'éclaircissement. Les réunions mensuelles sont un moment important pour les mentores, ce cadre leur permet de renforcer leurs compétences et d'apprendre les unes des autres.

Ces réunions peuvent inclure une mise à jour des réunions de l'espace sûr du mois précédent, les succès, les défis, les solutions proposées, les expériences de visites à domicile, l'examen des sessions du curriculum du mois précédent, la collecte d'exemples de réussite, l'aperçu des outils de participation, la formation pour les sessions à venir et l'examen de la soumission des rapports mensuels. 30

\section{ÉTUDE DE CAS - BALIKA RENFORCER LA CAPACITÉ DES MENTORES GRÂCE À DES SÉANCES HEBDOMADAIRES DE DÉMONSTRATION SIMULÉE}

BALIKA (Association bangladaise pour les compétences de la vie courante, les revenus et les connaissances des adolescents) était un projet de Population Council qui a fourni un soutien à l'éducation, une formation aux compétences de la vie courante et une formation aux moyens de subsistance par le biais de groupes hebdomadaires de AJF dirigés par des mentores féminins. Étant donné le rôle des mentores dans l'animation des sessions de groupes de AJF, il était essentiel de leur fournir une formation et un soutien continu.

Lors de la mise en œuvre du programme, BALIKA a utilisé un outil appelé " séances de démonstration simulées " où, chaque semaine, sept à huit mentores de sous-districts voisins se réunissaient avec leur superviseur dans les bureaux du programme pour s'entraîner à animer leurs séances pour la semaine suivante. Ces réunions en petits groupes ont permis d'améliorer la qualité des sessions et les compétences des mentores en matière d'animation, ainsi que de leur fournir un retour d'information régulier de la part des pairs et des superviseurs afin de présenter les sujets de BALIKA de manière plus efficace. Les réunions ont servi à l'analyse continue des performances des mentores et ont permis à l'équipe du programme d'identifier et d'inviter les leaders à représenter le groupe lors d'événements locaux et nationaux. Les réunions hebdomadaires ont également donné aux mentores l'espace et le temps de mieux se connaître, de se soutenir mutuellement, d'apprendre les unes des autres comment faire face aux défis de la mise en œuvre sur le terrain et de soulever les problèmes d'une voix collective.

Avec la mise en place de BALIKA, les experts locaux tels que les prestataires de soins de santé et les fournisseurs de services informatiques utilisent des sessions de démonstration hebdomadaires simulées pour dispenser une formation spécialisée dans les domaines de programme pertinents. L'équipe de BALIKA s'est rendu compte que le modèle de réunions hebdomadaires fonctionne de manière satisfaisante lorsque les mentores vivent à proximité les unes des autres et du bureau du programme. Si elles doivent parcourir de longues distances pour se rencontrer chaque semaine, le modèle peut ne pas être aussi efficace et doit être adapté.

Pour plus d'informations: Delaying child marriage through community-based skills-development programs for girls: Results from a randomized controlled study in rural Bangladesh. 2016. https://knowledgecommons.popcouncil.org/departments sbsr$\operatorname{pgy} / 557 /$.

30 Adapté de "Valoriser le potentiel des mentores: Recrutement, formation, et soutien des mentores pour des programmes dédiés aux adolescentes" du Population Council https://www.popcouncil.org/uploads/pdfs/2019PGY_MentorToolkit_fr.pdf 


\section{Opportunités d'autonomisation économique pour la mentore}

Des mentores compétentes et motivées sont essentielles au succès de tout programme d'espace sûr. Certaines stratégies visant à maintenir la motivation des mentores et leur volonté de continuer à jouer leur rôle impliquent la création d'opportunités d'autonomisation économique pour la mentore. Comme nous venons de le décrire, les rencontres programmées des mentors peuvent également leur être bénéfiques en leur donnant l'occasion d'organiser et de créer des groupes d'épargne, des initiatives de renforcement de compétences, et des possibilités de générer des revenus.

\section{ÉTUDE DE CAS - ABRIENDO OPORTUNIDADES UNE STRUCTURE DURABLE VIA DES ONG DIRIGÉES PAR DES MENTORS}

Abriendo Oportunidades $(\mathrm{AO})$ réalise des investissements essentiels dans les jeunes filles indigènes rurales âgées de 8 à 19 ans au Guatemala pour les aider à réussir leur transition d'adolescente en créant des réseaux de soutien social, en les mettant en contact avec des modèles et des mentors, en développant une base de compétences de vie et de leadership et en engageant la communauté à sensibiliser aux droits et aux besoins des filles. Les mentors $\mathrm{AO}$ sont des jeunes femmes âgées de 18 à 25 ans qui vivent dans les mêmes communautés que les groupes de filles.

Après avoir terminé le programme AO dans les hauts plateaux de l'ouest du Guatemala, le Population Council a guidé les anciennes participantes de l'AO devenues mentores communautaires à explorer la possibilité de créer une organisation non gouvernementale (ONG). En 2013, 15 mentores de I'AO ont créé une ONG qui met en place des groupes de filles de I'AO dans le cadre du programme plus large de I'AO, recevant une sous-compensation du Council pour faire le travail, en plus de gérer des groupes de filles indépendamment de la programmation du Council.

Cette ONG dirigée par des mentores a également un programme de génération de revenus dans le cadre duquel les membres produisent de l'artisanat pour le vendre et soutiennent les activités des groupes de filles. En 2016-2017, un groupe de mentores de I'AO de la région nord-est du Guatemala a créé sa propre organisation.

Cette organisation a été lancée par 18 mentores de l'AO, et elle est devenue une organisation de 25 membres. Suivant le modèle de l'AO, cette ONG facilite 12 groupes de filles dans la communauté. L'ONG a rédigé une proposition de financement d'activités visant à renforcer leur collaboration et leur coordination avec le gouvernement local. Elles prennent également des mesures pour aborder la question de la nutrition dans leur communauté en travaillant avec une autre ONG pour créer des jardins familiaux avec des plantes endémiques hautement nutritives.

Le Population Council a contribué à la création des deux organisations en leur fournissant des conseils juridiques et financiers, des conseils sur le meilleur type d'ONG à créer compte tenu des diverses exigences en matière de collecte de fonds et de responsabilité du gouvernement pour chaque catégorie d'ONG au Guatemala. Grâce au soutien du Population Council pour la collecte de fonds, les deux organisations ont reçu des fonds de donateurs privés et d'ONG internationales. La plate-forme des ONG a permis aux mentores de I'AO de se développer personnellement et professionnellement en tant que jeunes femmes. La formation de ces ONG a permis aux mentores d'élargir leurs compétences en matière d'animation et de leadership, de développer leurs compétences en matière de défense des intérêts de la communauté, de renforcer leurs réseaux de soutien social, d'améliorer leur exposition aux opportunités professionnelles dans leurs communautés, de retarder le mariage et d'éviter la migration.

Pour plus d'informations : Abriendo Oportunidades (“Ouverture des opportunités"), https://www.popcouncil.org/research/ abriendo-oportunidades-opening-opportunities. 


\section{Leadership en cascade / aspirations à la promotion}

Un modèle de développement de leadership couronné de succès dans de nombreux contextes du monde est celui où des adolescentes plus âgées de la communauté dans laquelle le programme opère sont formées comme mentores de leurs cadettes. L'approche peut se réaliser sur une période prolongée, tandis que les AJF suivent et achèvent elles-mêmes les activités du programme et apprennent à assumer des positions de leadership de plus en plus élevées, prenant notamment la tête des groupes de nouvelles inscrites. Dans la plupart des cas, le programme ne dispose cependant pas d'un temps initial suffisant pour permettre aux AJF de suivre un cycle complet de développement de leadership, et il est nécessaire de former spécifiquement un groupe d'adolescentes plus âgées au rôle de premières mentores.

Quel est donc le principe fondamental du modèle de leadership en cascade ? II s'agit de travailler avec des adolescentes plus âgées ou des jeunes femmes et d'en faire des leaders, responsables des programmes proposés à leurs cadettes.

- Une approche consiste à sélectionner un groupe de AJF plus âgées aux aptitudes prometteuses, originaires de la même communauté défavorisée, et de les soumettre à une formation intense de préparation au rôle de mentore.

- L'autre approche, plus exigeante en temps, consiste à amener les AJF, au fil des programmes qu'elles suivent elles-mêmes, à assumer des responsabilités de leadership. 


\title{
ÉTUDES DE CAS - LEADERSHIP EN CASCADE
}

\author{
Un exemple d'application du modèle de leadership en cascade est présenté ci- \\ dessous, cet exemple a découlé naturellement (" organiquement ") de la \\ croissance du programme.
}

\section{BINTI PAMOJA}

Le centre Binti Pamoja - Binti Pamoja était un programme destiné aux adolescentes de 10 à 19 ans vivant dans le bidonville de Kibera à Nairobi (Kenya). Le programme s'est développé autour d'un groupe initial de $15 \mathrm{AJF}$, divisé en deux groupes. Ces AJF ont grandi avec le programme. Au terme de deux ans au sein du programme, il est apparu clair qu'elles étaient prêtes à " recevoir leur diplôme " et à assumer des rôles de leadership, alors même que le programme était prêt à s'étendre dans la communauté. En 2006, la promotion initiale du programme a ainsi suivi une formation appelée à renforcer les compétences des AJF en matière d'animation de rencontre, de développement de groupe, de communication et de résolution de conflits. Les AJF ont été par la suite affectées, deux par deux, à la formation de leurs propres groupes de AJF dans la communauté. Les mentores/anciennes élèves ont assumé la responsabilité du recrutement des AJF, de la recherche d'un espace de rencontre au sein de la communauté, de la planification et animation des rencontres hebdomadaires, du soutien et conseil apportés aux AJF, et de la gestion du budget mensuel du groupe. Ces anciennes élèves ont touché un petit salaire et ont participé chaque mois à des réunions d'encadrement avec le personnel de Binti Pamoja.

En un an, le "Programme des Espaces sûrs " de Binti Pamoja comptait 20 anciennes élèves et 10 groupes. Devenu trop grand pour ses deux responsables originales, le programme a engagé deux anciennes élèves au titre d'agents de terrain à temps partiel, les chargeant de l'encadrement de celles responsables des espaces sûrs et de leurs groupes respectifs. Le programme a continué à croître, d'année en année, tandis que de nouvelles AJF achevaient le cycle principal de Binti, devenaient à leur tour anciennes élèves et formaient leurs propres groupes dans leur communauté. Dès 2009 , il comptait 50 anciennes élèves et 25 groupes et devenait, une fois encore, trop vaste pour ses deux agents de terrain. Un nouvel échelon de leadership a donc été introduit entre les anciennes élèves/leaders de groupe et les agents de terrain : les coordinatrices de village. Au nombre de cinq, celles-ci étaient responsables, chacune, d'un secteur géographique précis de Kibera. Elles supervisaient chacune quatre à six groupes de AJF. Elles aidaient les anciennes élèves de leur secteur à recruter, à identifier les espaces de rencontre appropriés, à superviser le contenu abordé par le groupe et à planifier les événements et les formations.

Suivant le modèle du leadership en cascade, Binti Pamoja a ainsi pu grandir, d'un programme initial de $40 \mathrm{AJF}$ se réunissant en un seul emplacement de Kibera à un programme de plus de $1000 \mathrm{AJF}$, composé de 30 groupes (de 15 à 25 AJF chacun), 75 anciennes élèves assumant un rôle de leadership ou l'autre, avec une présence dans chaque village de Kibera.

(Adapté de : "Conception d'un programme centré sur les AJF ; Boîte à outils pour l'élaboration, le renforcement et l'expansion de programmes destinés aux adolescentes ", du Population Council.) 


\section{DES NORMES MINIMALES POUR LE CONTENU PROGRAMMATIQUE "APPROPRIÉ "}

\section{Un contenu programmatique approprié pour des espaces sûrs afin de développer des "compétences" et des aptitudes à la vie quotidienne pertinentes}

Dans SWEDD I, l'intention était de séparer les AJF en deux tranches d'âge pour les espaces sûrs et aussi avec la création des espaces sûrs communautaires et des espaces sûrs scolaires, démontrant ainsi une segmentation par statut de scolarisation des AJF.

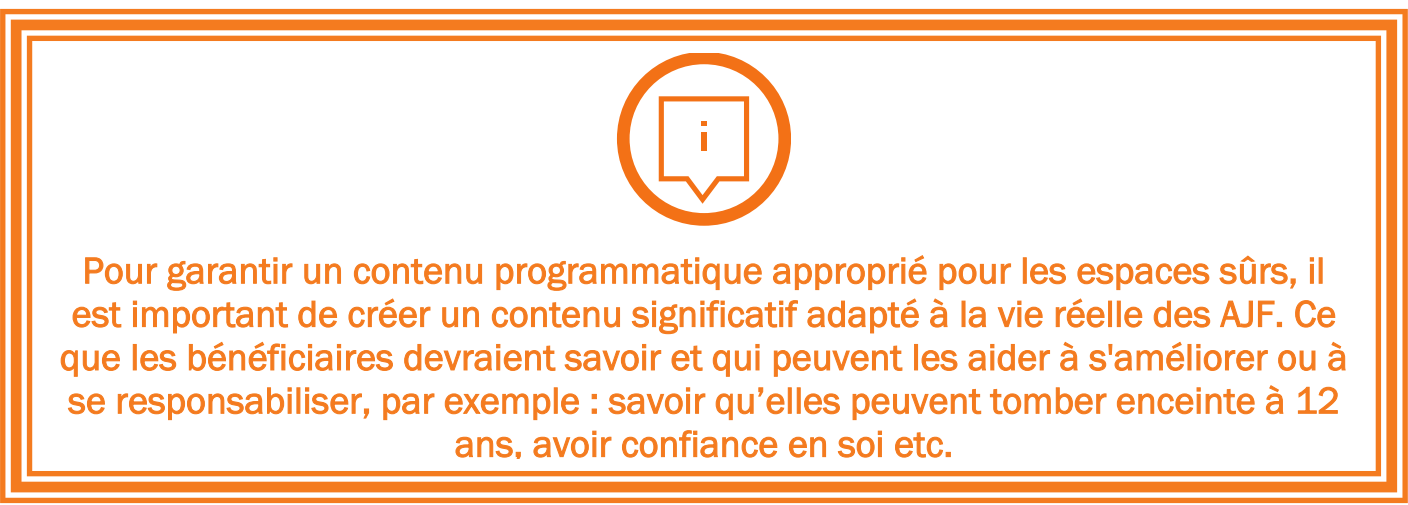

Le contenu des programmes destinés aux adolescentes est souvent trop généralisé. II n'est pas adapté à des populations spécifiques, à savoir qu'il ne reconnaît pas l'hétérogénéité des AJF et présente la vie des AJF comme trop rose. Il est présomptueux de croire que le matériel en question est initialement destiné à atteindre des populations plus privilégiées. Pour qu'un programme réussisse, le contenu doit être pertinent pour les AJF ciblées par SWEDD et doit prioriser des sujets en accordance avec les besoins des segments d'AJF ciblés ; cela peut se réaliser par le biais de la construction et le renforcement des compétences. ${ }^{31}$

\section{Un ensemble essentiel d'activités pertinentes pour les espaces sûrs du SWEDD}

Pour s'assurer que les AJF et leur communauté trouvent un programme utile et digne de leur temps, il est important qu'elles le considèrent comme une valeur ajoutée à leur vie et à leur communauté. L'ensemble constitutif des activités doit être basé sur des preuves et faire partie de la théorie du changement du programme liant les activités et les interventions aux résultats et aux objectifs. Il est donc indispensable de créer des compétences qui peuvent être utiles aux AJF audelà de leur participation aux sessions des espaces sûrs.

La combinaison de différents types de compétences crée des synergies et peut accroître l'utilité et la pertinence du AJF. A titre d'exemple, nous pouvons renforcer les connaissances de l'AJF en matière de recherche de soins de santé, accroître la demande de services de santé, fournir un soutien social pour surmonter les craintes et les hésitations, et inclure des stratégies de microépargne pour aider l'AJF à choisir et à accéder aux services de santé. (Pour plus d'informations sur

${ }^{31}$ Adapté de "Intentional design: reaching the most excluded girls in the poorest communities: A Guide for Practitioners and Advocates" du Population Council 


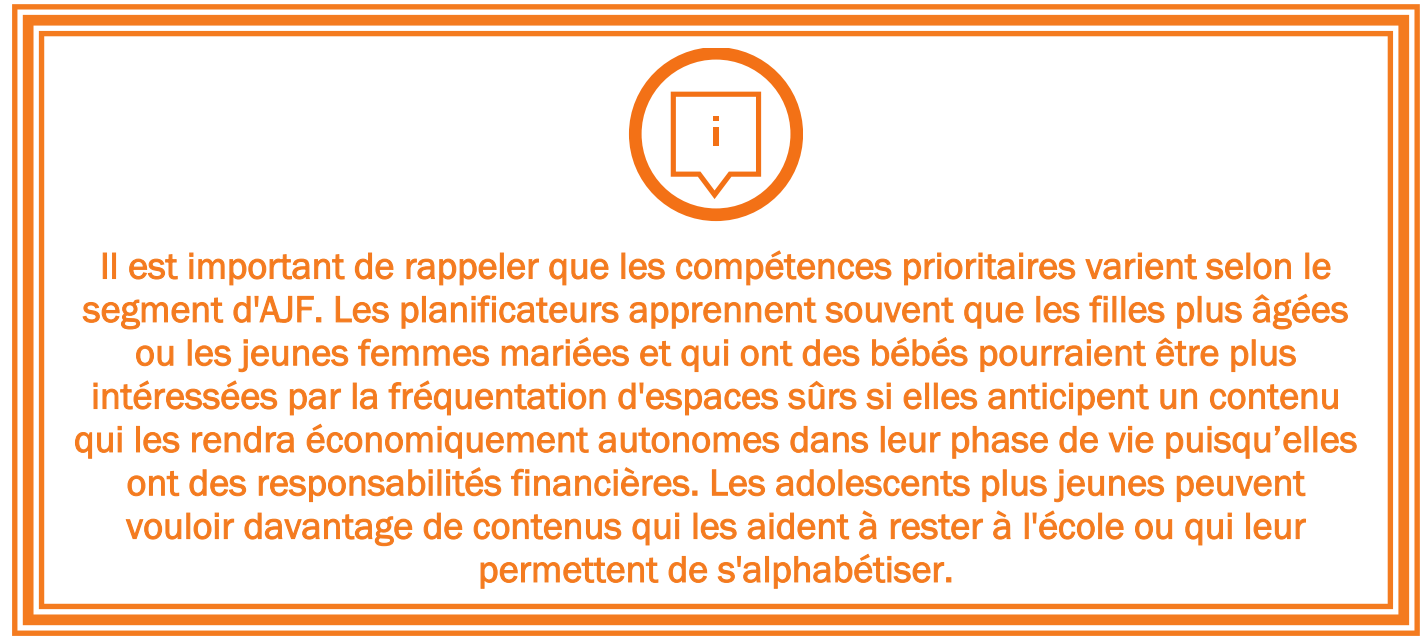

Une compétence est une réserve de valeur - qui peut être concrète ou abstraite - que les programmes renforcent afin de responsabiliser les AJF, et leur permettre de réduire les risques auxquels elles sont confrontées et d'élargir leurs possibilités. À titre d'exemple, les programmes contribuent à fournir et renforcer des compétences et des ressources de protection telles que : des cartes d'identité, une amélioration de l'efficacité personnelle dans l'utilisation du préservatif, un renforcement des notions d'arithmétique, de même qu'un soutien social sur lequel les AJF peuvent s'appuyer pour négocier et réduire les risques quotidiens ou protéger leur épargne. Les compétences de protection peuvent être de nature humaine, sociale, économique et cognitive. ${ }^{32}$

Tableau 4: Types de compétences de protection que les AJF peuvent renforcer grâce à leur participation à un espace sûr 33

\begin{tabular}{|c|c|c|}
\hline $\begin{array}{l}\text { Les Types de } \\
\text { compétences }\end{array}$ & Explications des types de compétences & $\begin{array}{l}\text { Illustrations des types de } \\
\text { compétences }\end{array}$ \\
\hline $\begin{array}{l}\text { Les compétences } \\
\text { sociales }\end{array}$ & $\begin{array}{l}\text { Les réseaux auxquels les AJF peuvent } \\
\text { accéder pour les aider à tirer parti des } \\
\text { opportunités. Espaces sûrs pour les AJF, le } \\
\text { soutien social, avoir au moins trois amies } \\
\text { n'appartenant pas à la famille, l'accès au } \\
\text { mentorat (guide, conseiller), le } \\
\text { développement des compétences de vie } \\
\text { (la prise de décision, la négociation, } \\
\text { l'établissement de relations avec les } \\
\text { autres). }\end{array}$ & 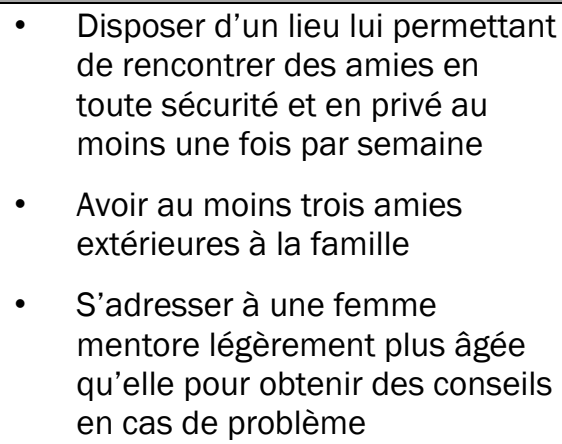 \\
\hline
\end{tabular}

\footnotetext{
32 Adapté de“ développer les compétences de protection des AJF : Ensemble d'Outils pour la Conception d'un Programme " du Population Council

33 Adapté de "développer les compétences de protection des AJF : Ensemble d'Outils pour la Conception d'un Programme " du Population Council
} 


\begin{tabular}{|c|c|c|}
\hline $\begin{array}{l}\text { Les Types de } \\
\text { compétences }\end{array}$ & Explications des types de compétences & $\begin{array}{l}\text { Illustrations des types de } \\
\text { compétences }\end{array}$ \\
\hline $\begin{array}{l}\text { Les compétences } \\
\text { cognitives }\end{array}$ & $\begin{array}{l}\text { Compétences souvent nourries à l'école } \\
\text { (et idéalement au-delà). Mathématiques, } \\
\text { l'alphabétisation }{ }^{34} \text {, la confiance et l'auto- } \\
\text { expression claire, l'analyse critique, la } \\
\text { communication et la résolution de } \\
\text { problèmes. }\end{array}$ & $\begin{array}{l}\text { - Savoir demander à une autorité } \\
\text { féminine si elle est mal à l'aise } \\
\text { avec un homme } \\
\text { - } \quad \text { Avoir réfléchi attentivement aux } \\
\text { compétences nécessaires qui lui } \\
\text { permettraient d'avoir un emploi } \\
\text { qui lui plaît } \\
\text { - Posséder des compétences de } \\
\text { négociation et de résolution des } \\
\text { problèmes pour affirmer son } \\
\text { souhait de poursuivre sa } \\
\text { scolarité }\end{array}$ \\
\hline $\begin{array}{l}\text { Les compétences } \\
\text { humaines/santé }\end{array}$ & $\begin{array}{l}\text { Les choses qui aident à protéger la vie des } \\
\text { AJF et celle de ceux qui les entourent. Les } \\
\text { connaissances sur la santé sexuelle et } \\
\text { reproductive (y compris les infections } \\
\text { sexuellement transmissibles), la recherche } \\
\text { d'une clinique de santé, des signes de } \\
\text { danger pendant la grossesse et le travail, } \\
\text { la façon de traiter un jeune enfant } \\
\text { souffrant de diarrhée (pour les AJF qui } \\
\text { prennent soin des enfants plus jeunes) }\end{array}$ & $\begin{array}{l}\text { - Connaître le lieu où se trouvent } \\
\text { les centres de secours dans la } \\
\text { communauté } \\
\text { - Connaître les caractéristiques de } \\
\text { la menstruation et comment les } \\
\text { gérer de manière sûre et } \\
\text { hygiénique } \\
\text { - Connaître les mutilations } \\
\text { génitales féminines (comment et } \\
\text { à quel moment elles sont } \\
\text { pratiquées, comment aider une } \\
\text { personne menacée de MGF, et } \\
\text { savoir qu'elles sont illégales) } \\
\text { Savoir à quel moment se laver } \\
\text { les mains et comment le faire } \\
\text { correctement dans la vie } \\
\text { quotidienne et dans le contexte } \\
\text { des épidémies et des maladies } \\
\text { infectieuses }\end{array}$ \\
\hline
\end{tabular}

34 Dans le cadre du SWEDD I, l'axe des sessions modulaires intègre l'alphabétisation. En effet, les adolescentes bénéficient aussi de cours d'alphabétisation pour savoir lire, écrire et calculer, de l'établissement d'actes de naissance si elles n'en ont pas et d'un bilan de santé. Ce paquet d'activités doit conduire les adolescentes à acquérir des connaissances en vue de changer de comportement et à les utiliser pour acquérir l'estime et la confiance en elles, pour leur propre éveil afin de se prémunir contre les mariages et les grossesses précoces et d'améliorer leur état de santé. Elles sont fortement encouragées à fréquenter les centres de santé et à disposer des documents d'état civil. En outre, les adolescentes doivent avoir les aptitudes à lire, compter et calculer pour développer des activités économiques et financières susceptibles de contribuer à favoriser leur autonomie par l'entreprenariat. Telle est la condition pour que les adolescentes et les jeunes femmes puissent vivre dignement en réduisant leur dépendance sociale et économique.

Le programme d'alphabétisation est mis en place avec l'appui du Ministère en charge de l'alphabétisation. Un instructeur est commis par les services compétents. II existe un programme d'alphabétisation standardisée. Les séances se tiennent en alternance avec les sessions éducatives soit deux ou trois fois par semaine sur une période de six mois. 


\begin{tabular}{|c|c|c|}
\hline $\begin{array}{l}\text { Les Types de } \\
\text { compétences }\end{array}$ & Explications des types de compétences & $\begin{array}{l}\text { Illustrations des types de } \\
\text { compétences }\end{array}$ \\
\hline $\begin{array}{c}8 y^{2}=0 \\
\text { Les } \\
\text { compétences } \\
\text { économiques }\end{array}$ & $\begin{array}{l}\text { Des compétences spécifiques qui aident les } \\
\text { AJF à planifier pour l'avenir. Éducation } \\
\text { financière selon l'âge, sachant comment } \\
\text { créer un budget simple (établir un compte } \\
\text { d'épargne), et la connaissance sur les } \\
\text { opportunités de formation pour le travail. }\end{array}$ & $\begin{array}{l}\text { - } \begin{array}{l}\text { Avoir des épargnes qu'elle peut } \\
\text { utiliser en cas d'urgence } \\
\text { personnelle ou familiale }\end{array} \\
\text { - } \quad \text { Avoir un plan pour se rendre à } \\
\text { la banque la plus proche ou } \\
\text { dans tout autre établissement } \\
\text { de services financiers avec des } \\
\text { amies ou un parent } \\
\text { - } \quad \text { Posséder les compétences et la } \\
\text { confiance nécessaires pour } \\
\text { créer un budget et assurer le } \\
\text { suivi des revenus et des } \\
\text { dépenses } \\
\text { - Posséder une compétence } \\
\text { productive permettant de } \\
\text { gagner de l'argent } \\
\text { Avoir les documents } \\
\text { personnels nécessaires pour } \\
\text { accéder aux services financiers }\end{array}$ \\
\hline
\end{tabular}

\section{Des compétences de vie adaptées à l'âge pour les AJF}

Dans le cadre du paquet de base, il est important de vérifier si le contenu est approprié et dans le cas contraire l'adapter en conséquence. Ainsi il est nécessaire d'identifier au préalable la connaissance et les " compétences de protection " dont les AJF ont besoin et veulent pour réduire de manière adéquate et durable les risques. Cela est d'autant crucial que différents segments ont des besoins prioritaires différents pour réduire les risques de mariage d'enfants, de grossesse précoce, et d'abandon scolaire.

Par conséquent, une fois que vous avez déterminé quelles compétences créer dans vos espaces sûrs, la prochaine étape consiste à décider de qui bénéficiera de quelles compétences. Il est vrai que le choix du contenu ne vous appartient pas toujours intégralement. De nombreux programmes sont préconçus ou limités aux activités prédéfinies.

Quant au projet SWEDD, le développement des compétences de vie et en santé, de même que le développement de tout autre type de compétence s'organisent en vingt-six (26) sessions de formation regroupée dans les trois domaines suivants :

- La santé sexuelle et reproductive : la puberté, la reproduction, la menstruation et l'hygiène de la jeune fille, les IST y compris et VIH/Sida, la planification familiale, la nutrition, les grossesses, l'accouchement et l'avortement.

- Le genre et les droits humains : le genre, les droits des AJF, les violences basées sur le genre, les risques liés à la mutilation génitale féminine, les risques liés au mariage d'enfant et à la grossesse précoce.

- Les compétences de vie : l'estime en soi et la conscience en soi, les valeurs, la capacité à s'identifier et à gérer ses émotions, les relations saines et malsaines, la résolution de problèmes et de conflits, la négociation, les relations interpersonnelles et la capacité à dire "NON", les techniques de refus (pression de la part de l'entourage), les capacités de communication et d'écoute, l'aptitude à se fixer des objectifs et à travailler en équipe, à servir d'exemple et à prendre une décision motivée. 
En revanche vous avez la possibilité de prendre des décisions, dans les limites de ces contraintes, sur l'objet précis de votre concentration, d'autres éléments à inclure, ainsi que sur les groupes d'âge et l'ordre dans lequel vous allez présenter vos thèmes.

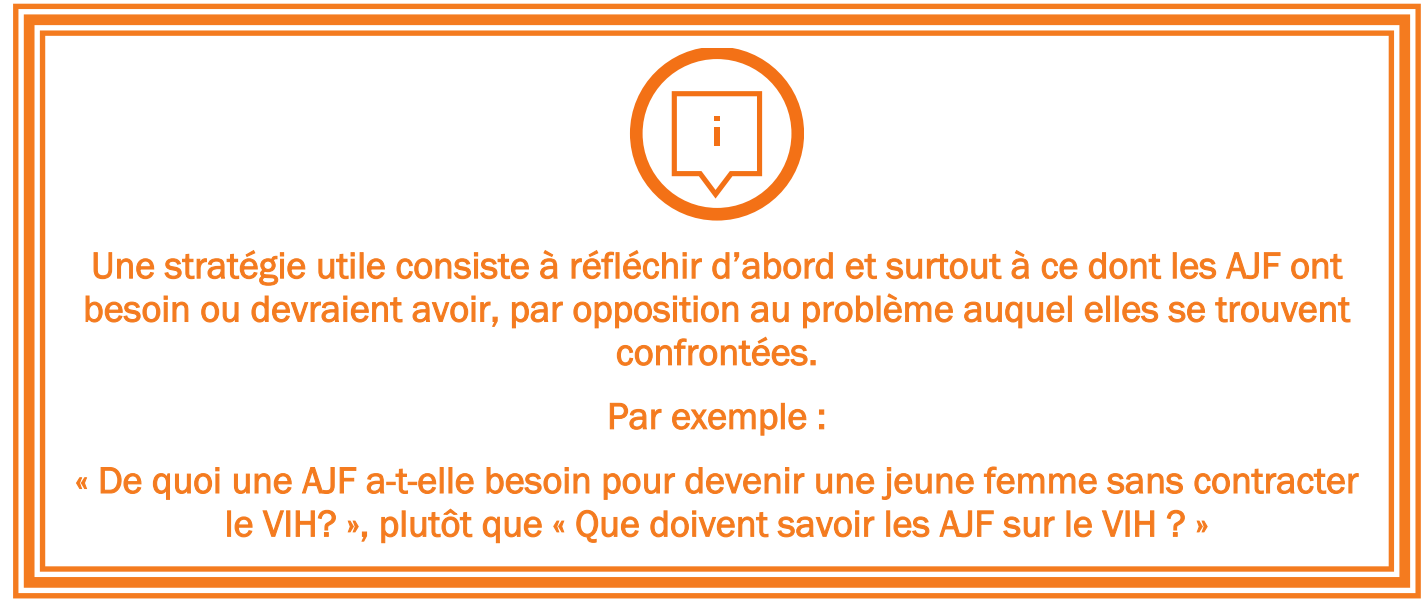

L'exercice de " cadre de création de capital 35 " pourrait vous aider à réfléchir à toutes les formes de "capital " - compétences et ressources - dont une fille a besoin pour effectuer sainement sa transition vers l'âge adulte. À défaut d'une activité particulière, cette approche représente plutôt un mode de réflexion.

Les compétences de la plus grande importance peuvent être mises en valeur ainsi que l'âge auquel elles sont nécessaires. Pensez aux AJF dans votre programme et referez-vous à la figure 7. Qui sont-elles ? Quel âge ont-elles ? Que font les AJF dans la communauté et que veulent-elles? Quand est-ce

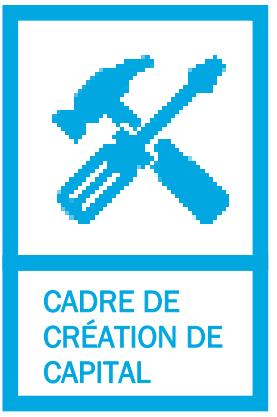
qu'elles ont un besoin particulier de connaissances, de compétences, ou de ressources ? II se pourrait que votre programme comporte des travailleuses domestiques de 10-14 ans ou des jeunes adolescentes avec des bébés. Existe-t-il des compétences qui sont particulièrement importantes pour ce segment de AJF ? Par exemple, si vous travaillez avec une population mobile (nomade), est-il primordial pour eux d'avoir un compte d'épargne ? Est-il possible d'adapter les compétences aux besoins de votre population particulière ?

${ }^{35}$ Adapté de "Conception d'un programme centré sur les AJF : Boîte à outils pour l'élaboration, le renforcement et l'expansion de programmes destinés aux adolescentes "

https://knowledgecommons.popcouncil.org/cgi/viewcontent.cgi?article=1879\&context=departments sbsr -pgy du Population Council 

d'AJF

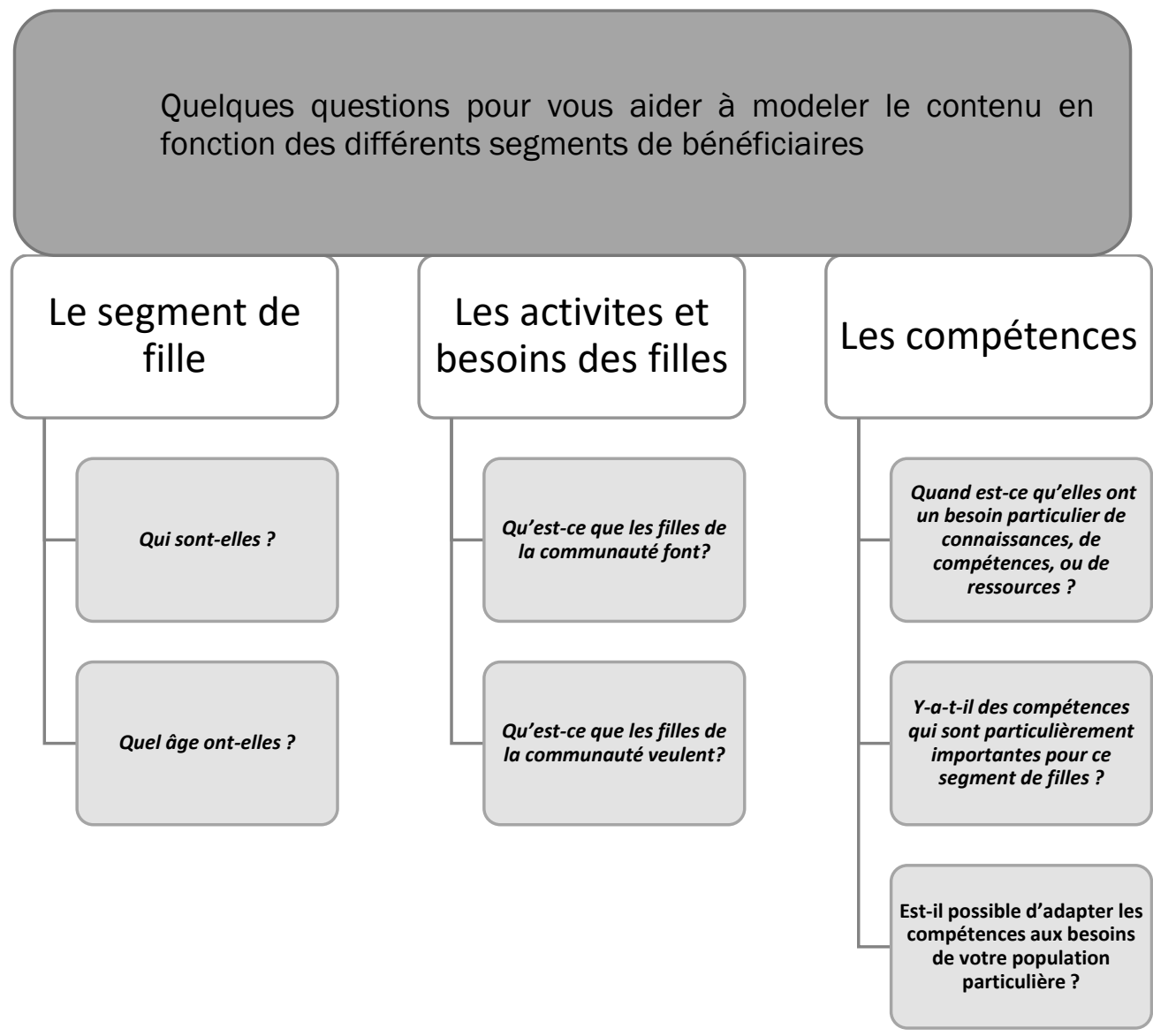

Après avoir répondu à ces questions, vous pouvez avoir une discussion sur les activités du programme qui aideront les AJF à obtenir les compétences dont elles ont besoin et au moment où elles en auront besoin.

L'exercice de renforcement des compétences ${ }^{36}$ exige des parties prenantes qu'elles répondent à des questions importantes liées à la situation des AJF dans leur contexte, notamment :

- Quelles sont les notions qu'une fille doit connaître à l'âge de 12 ans ?

- Quelles compétences une fille a besoin pour faire face aux pressions auxquelles elle est confrontée?

- Qu'est-ce qu'une fille doit être capable de faire pour relever ces défis? Cet exercice doit définir des objectifs réalisables à court terme et moins large au niveau des AJF, par exemple, "sortir de la pauvreté ", et moins vagues et négatifs que de simplement demander aux AJF " d'éviter la grossesse. "

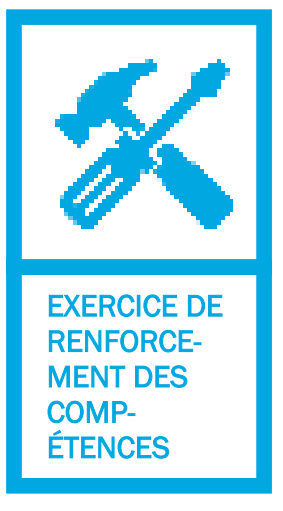

36 Bruce, Judith, Sarah Engebretsen, et Kimberly Glazer. 2016. "Boîte à Outils: Renforcement des Compétences.” Traduction française. New York: Population Council. https://knowledgecommons.popcouncil.org/cgi/viewcontent.cgi?article=1666\&context=departments sbsr -pgy 
L'exercice implique que les décideurs des programmes, les mentores potentielles et les AJF ellesmêmes mènent une nouvelle réflexion sur ce qui est essentiel à leurs yeux, et aident les différentes parties prenantes à réfléchir aux compétences qui peuvent améliorer la capacité des AJF à mieux gérer les défis et les opportunités au jour le jour et à identifier les contenus de programmes les plus significatifs. 37

Il est déterminant dans la mesure où l'exercice de renforcement des compétences correspond à une "vision renouvelée " des programmes consacrés aux AJF et peut impliquer les parties prenantes à différents niveaux et selon différents points de vue. Ensemble, ces opinions génèrent un dialogue fondé sur des données probantes."

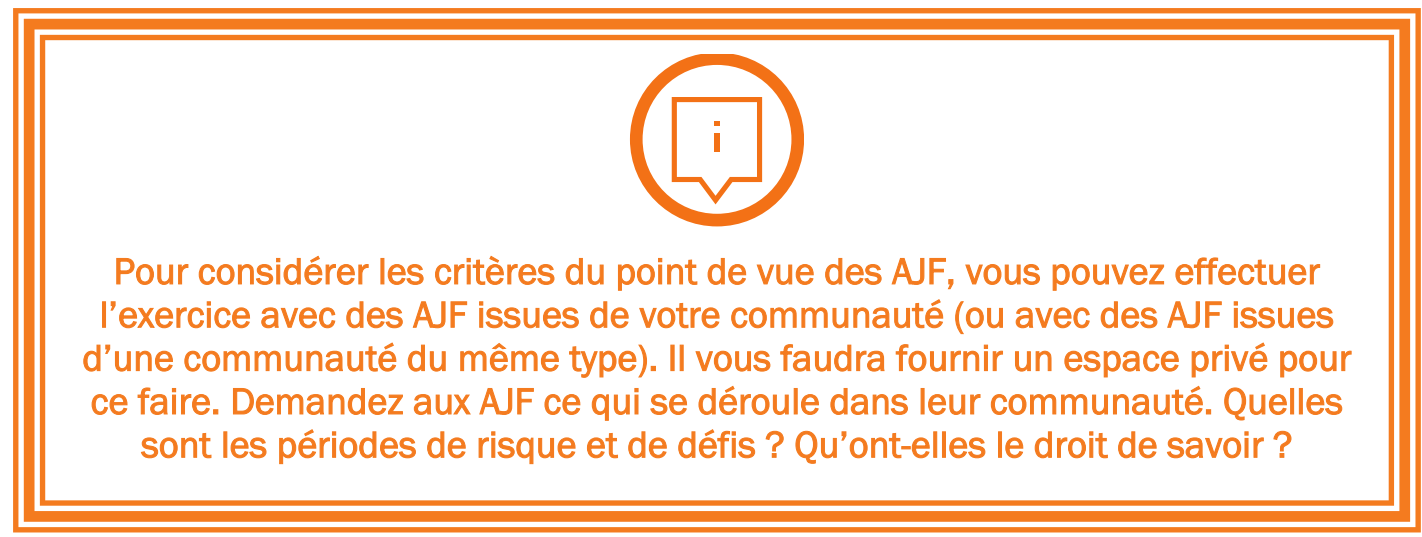

En Haïti, par exemple, les AJF âgées de 10 à 14 ans vivant dans un camp de personnes déplacées après le tremblement de terre de 2010 estimaient qu'il était important de porter à la connaissance des AJF dès l'âge de 6 ans les risques de se promener dans le camp avec les jeunes garçons qui flirtent avec des jeunes AJF. Leur calendrier est-il différent de ce que les responsables du programme avaient prévu ?38

En une journée (ou moins), un outil tel que décrit ici pourrait vous aider à établir une liste des compétences par segment (âge, géographie, l'état matrimonial, ou un mélange). Elles peuvent être divisées en 10-20 compétences principales (celles qui sont d'une importance cruciale et que vous avez décidé d'inclure) et un autre groupe de compétences "souhaitables " (celles qui sont importantes comme un objectif atteignable). II y a souvent (mais pas toujours) un accord sur ce que ces groupes devraient être. Néanmoins, une fois terminée, vous aurez une liste de compétences qui peuvent servir de base pour développer un contenu adapté aux segments différents des AJF dans votre programme. 39

\footnotetext{
37 Adapté de "Boîte à Outils : Renforcement des Compétences/ développer des repères positifs pour les adolescentes " du Population Council

38 Adapté de "Boîte à Outils : Renforcement des Compétences/ développer des repères positifs pour les adolescentes " du Population Council

39 Adapté de "Boîte à Outils : Renforcement des Compétences/ développer des repères positifs pour les adolescentes " du Population Council
} 
Les AJF, même celles qui vivent dans la même communauté, constituent un groupe diversifié. Certaines ont des expériences et des situations similaires, comme le fait d'avoir 15 ans, de vivre avec leurs deux parents et d'aller à l'école, tandis que d'autres sont très différentes : elles sont déjà mariées à 15 ans, ne vont pas à l'école et vivent avec leur mari. C'est pourquoi il est nécessaire de segmenter les AJF dans les zones de vos programmes ou adapter un contenu spécifique à chaque segment. 40

Vous aurez tendance à comparer idéal et réalité, mais concentrez-vous sur l'âge auquel une fille devrait savoir ou avoir quelque chose. II est acceptable qu'elle connaisse ou possède ces compétences plus tôt, mais dans les circonstances actuelles ou normales (sombres comme il pourrait l'être) À quel âge devrait-elle savoir?

Pour décider des compétences dont chaque segment de AJF de votre programme a besoin il faut refléter sur les questions suivantes:

- À quel moment sont les AJF vraiment en danger du mariage précoce ou de la traite ?

- À quel âge doivent-elles se confronter à leur famille au sujet de leurs propres besoins (faim, trouver de la nourriture, défendre leurs droits, trouver des façons de gérer leur revenu)?

Vous pouvez utiliser la "Boîte à Outils : Renforcement des Compétences " du Population Council41 (décrit brièvement à la page 88) pour faciliter ce processus.

Tableau 5 : Les phases de l'adolescence 42

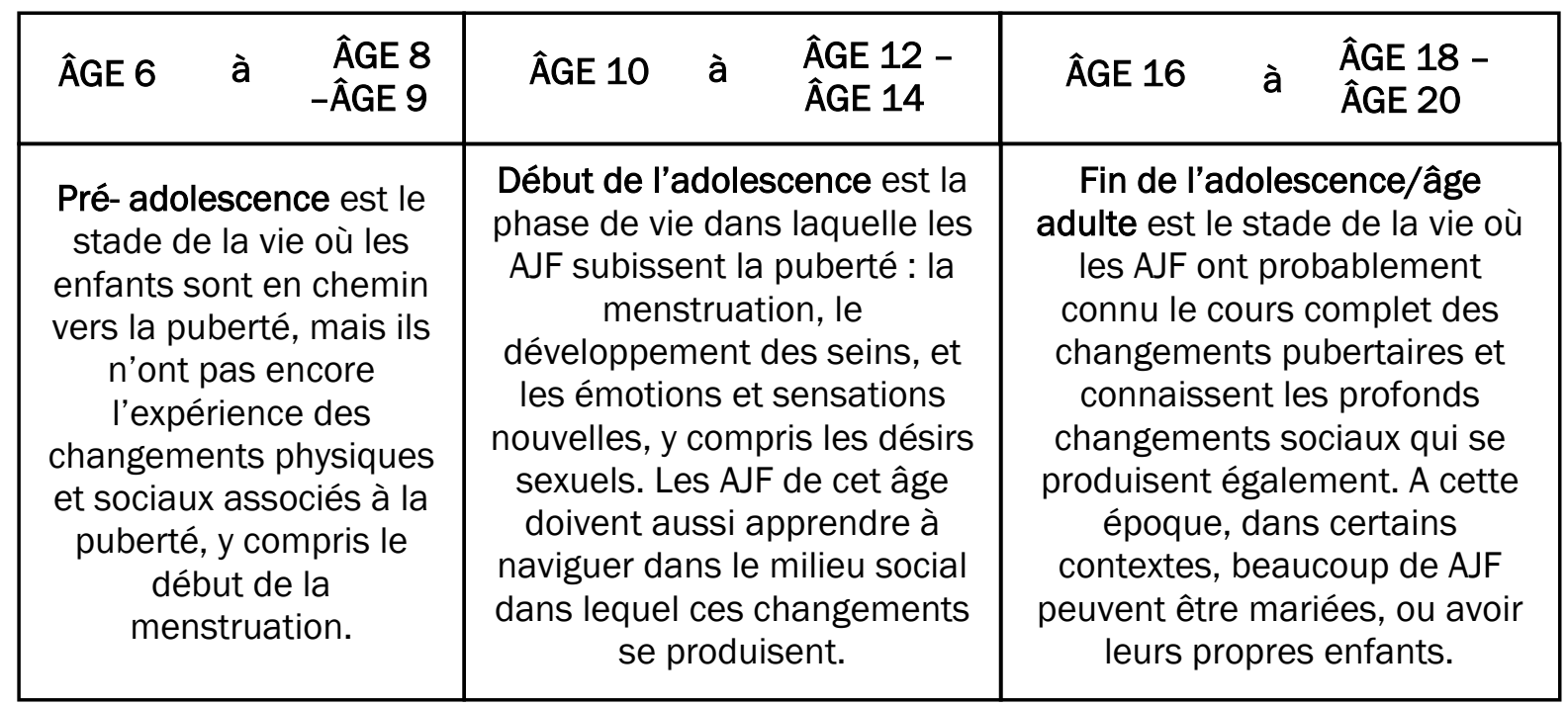

40 Adapté de "Intentional design: reaching the most excluded girls in the, poorest communities: A Guide for Practitioners and Advocates" du Population Council

${ }^{41}$ Adapté de "Boîte à Outils : Renforcement des Compétences/ développer des repères positifs pour les adolescentes " du Population Council

42 Adapté de "Boîte à Outils : Renforcement des Compétences/ développer des repères positifs pour les adolescentes " du Population Council 
Tableau 6 : Exemples de curriculums qui pourraient consultables en tant que projets devant être adaptés au contexte local du SWEDD.

\begin{tabular}{|c|c|}
\hline & $\begin{array}{l}\text { Leadership et mentorat des filles: Perspectives sur les programmes centrés } \\
\text { sur les filles } \\
\text { Ce document d'orientation sur le mentorat fournit un curriculum pour les } \\
\text { programmes centrés sur les filles. } \\
\text { https://www.popcouncil.org/uploads/pdfs/2012PGY GirlsFirst Leadership.pdf }\end{array}$ \\
\hline & $\begin{array}{l}\text { Un SEUL Programme } \\
\text { Ce curriculum fournit des directives et des activités pour les éducateurs afin } \\
\text { de fournir une approche unifiée de l'éducation à la sexualité, au genre, au VIH } \\
\text { et aux droits de l'homme. } \\
\text { Volume } 1 \text { : Guide } \\
\text { https://www.popcouncil.org/uploads/pdfs/2011PGY ItsAllOneGuidelines fr.pdf } \\
\text { Volume } 2 \text { : Activités } \\
\text { https://www.popcouncil.org/uploads/pdfs/2011PGY_ItsAllOneActivities_fr.pdf }\end{array}$ \\
\hline & $\begin{array}{l}\text { Santé et compétences de la vie courante pour les filles des zones rurales } \\
\text { Cet exemple de curriculum est issu de l'Initiative pour les adolescentes-Kenya } \\
\text { et est destiné aux adolescentes des zones rurales. II leur permet d'acquérir } \\
\text { des connaissances et des compétences sur des sujets tels que l'eau, } \\
\text { l'assainissement et l'hygiène, la nutrition et le VIH et le sida. } \\
\text { https://www.popcouncil.org/uploads/pdfs/2015PGY AGl- } \\
\text { K HealthLifeCurriculum Wajir.pdf }\end{array}$ \\
\hline $8 \mathrm{AGLKK \textrm {KB }}$ & $\begin{array}{l}\text { Éducation financière pour les filles des zones rurales } \\
\text { Ce curriculum type de l'Initiative pour les adolescentes - Kenya est destiné aux } \\
\text { adolescentes des zones rurales et couvre des sujets tels que les dépenses, } \\
\text { l'épargne, les possibilités de gagner de l'argent et la gestion du stress pour les } \\
\text { aider à passer de la dépendance économique à l'indépendance. } \\
\text { https://www.popcouncil.org/uploads/pdfs/2015PGY_AGl- } \\
\text { K FinancialCurriculum Wajir.pdf }\end{array}$ \\
\hline itr & ntered-program-resol \\
\hline
\end{tabular}

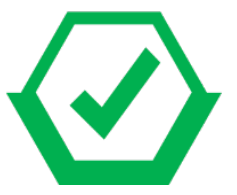

À FAIRE : Prendre le temps de réfléchir aux compétences et ressources dont les AJF du programme ont besoin, à quel âge, et déterminer celles auxquels le programme peut contribuer.

À ÉVITER : Supposer que les AJF de tous âges doivent toutes avoir les mêmes compétences et ressources 


\section{DES NORMES MINIMALES POUR LA MISE EN CEUVRE DES SESSIONS ENGAGEANTES ET DYNAMIQUES}

\section{Des mentores formés à l'utilisation de méthodes participatives pour encourager une participation énergique dans des groupes d'espaces sûrs.}

Utiliser des méthodes participatives est nécessaire pour engager et dynamiser les sessions de groupe. Par conséquent, la mentore doit connaître les AJF participant à l'espace sûr. Ceci est important d'autant plus qu'il peut être nécessaire de changer l'approche pour diriger les sessions, selon le groupe. A titre d'exemple, les AJF non scolarisées peuvent avoir des compétences en littératie inférieures à celles des AJF scolarisées. Pour les groupes à faible niveau d'alphabétisation, les mentores peuvent vouloir dessiner plus d'images et utiliser plus de symboles Iorsqu'ils écrivent sur le tableau.

Les mentores doivent préparer les séances en avance et utiliser un langage simple et s'assurer que les instructions sont claires avant de commencer toute activité. Aussi, elles ne doivent pas poser plus de quelques questions à la fin de chaque activité. II est important que les mentores travaillent avec les adolescentes où et comme elles sont, et non pas où ou comme la mentore pense qu'elles devraient être. Elles doivent également vérifier la compatibilité et

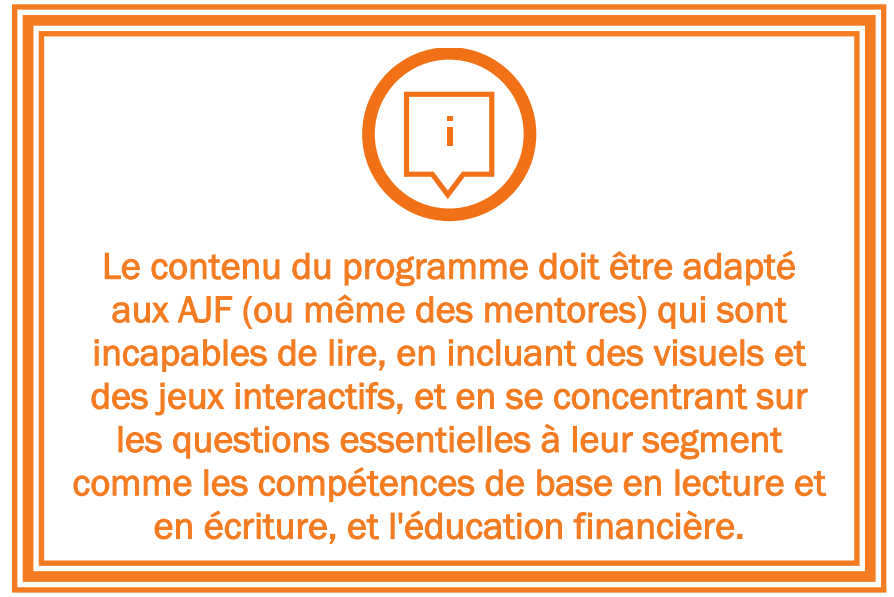
l'acceptabilité culturelles des séances. II convient de se familiariser avec les normes culturelles locales et adapter les séances en conséquence. ${ }^{43}$

De plus, l'utilisation de méthodes d'enseignement participatives, telles que la discussion de groupe, le brainstorming, les jeux de rôle, le travail en petits groupes, les jeux éducatifs et les contes sont d'excellents moyens d'impliquer les AJF dans la session (veuillez consulter le document SWEDD "Guide de la Mentore : Animatrice des 'espaces sûrs' "). Les mentores peuvent préférer donner une conférence et peuvent trouver l'animation d'un jeu ou d'un autre exercice interactif un peu difficile. Cependant, les jeux et les activités donnent aux participantes l'occasion d'interagir librement, et génèrent souvent des discussions qui ne peuvent être obtenues par d'autres méthodes. 


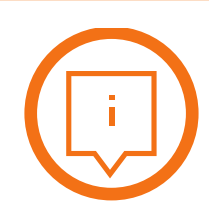

N'oubliez pas que les rencontres de groupe sont censées être un

environnement amusant et détendu, et NON un environnement qui ressemble à une salle de classe !
Les techniques participatives sont des moyens de s'exercer à utiliser de nouvelles compétences (ex., la communication et la prise de décision) pour les participantes. Imaginez une séance de formation active, parfois bruyante et amusante. Une autre façon de rendre les sessions d'apprentissage plus vivantes et engageantes est d'utiliser des aides visuelles (ex. une boite à image qui communique certains aspects des leçons). Alternativement, des objets réels peuvent être utilisés pour représenter des photos.

Des mentores formées pour appliquer les principes pratiques des sessions interactives centrées sur les participantes 44

1. Établir un contexte d'apprentissage basé sur l'égalité, le respect mutuel et les droits humains.

- Toutes les AJF doivent se sentir impliquées, écoutées, à l'aise et à l'abri du ridicule, plus particulièrement quand elles prennent le risque d'émettre de nouvelles idées.

- Encouragez la participation, en particulier parmi celles qui se sentent isolées ou intimidées.

- Les raisons de ces sentiments sont multiples : elles sont liées, notamment, aux différences de pertinence sociale associées au genre, à la classe sociale ou à l'âge, à la connaissance limitée de la langue parlée à l'école, ou au handicap physique ou autres besoins spécifiques.

- Les activités qui valorisent le respect d'autrui et l'esprit d'équipe, de même que le partage des occasions de leadership, favorisent la participation des AJF.

- En début de programme, invitez vos AJF à élaborer ensemble les directives, fondées sur le respect, qui guideront leur conduite dans l'espace sûr.

- Invitez les à respecter la vie privée les unes des autres et rappelez-leur de ne pas divulguer ce qu'elles estiment appartenir strictement à leur vie privée. N'oubliez pas que certaines AJF risquent d'en intimider ou même de maltraiter d'autres après la discussion de sujets sensibles. Rassurez les AJF que vous-même, leur mentore, garderez toutes les discussions confidentielles. Vous leur montrerez ainsi l'exemple du respect de la vie privée.

- Veillez à ce que les AJF comprennent bien qu'elles ont le droit de ne pas participer ou parler de leur expérience si l'idée les met mal à l'aise.

- Encouragez toutes les AJF à partager leurs pensées. Ne jugez pas les idées émises.

${ }^{44}$ Adapté de "Un Seul Programme. Volume 2 : Activités Pour Une Approche Pédagogique Unifié En De La Sexualité, Du Genre, Du Vih Et Des Droits Humains " 
2. Favoriser un processus qui tire parti du vécu des AJF, en intégrant de nouvelles informations et idées dans ce qu'elles savent ou pensent déjà.

- N'oubliez pas que toutes les participantes ont déjà une certaine connaissance et un certain vécu. Écoutez et posez les questions propices à leur exposition.

- Écoutez les inquiétudes exprimées et posez des problèmes hypothétiques qui reflètent la réalité de leur vie. Engagez-les dans la résolution de ces problèmes, en les amenant à opérer leurs propres choix et, ce faisant, à développer leurs idées.

- Encouragez vos AJF à explorer le sens concret, dans leur propre vie, d'un sujet abordé.

- Le rôle de la mentore devrait être comme celui d'un médiateur de débats sur les idées de vos AJF, plutôt que d'un professeur ex cathedra ou d'une simple source d'information. Au besoin, corrigez leurs erreurs factuelles ou aidez-les à reconnaître les commentaires qui manquent de respect.

- De temps à autre, il pourrait s'avérer utile de partager un épisode pertinent de votre propre expérience. Toutefois, veillez toujours à maintenir les limites appropriées par rapport à vos AJF.

3. Recourir à un vaste éventail de stratégies pour engager les AJF.

- Préparez différentes méthodes et approches adaptables aux besoins d'apprentissage de vos AJF.

- Si une leçon ou une activité ne vous est pas familière, commencez par lire attentivement l'information la concernant et passez soigneusement en revue les objectifs poursuivis. Préparez à l'avance tout le matériel nécessaire!

- Préparez un plan de rechange qui vous permet de faire aisément face aux circonstances imprévues (temps disponible, météo ou réaction des AJF, par exemple).

- Dans la mesure du possible, choisissez des activités amusantes !

4. Renforcer les capacités de réflexion, d'étude et d'analyse critique des AJF, sur leur propre vie et sur le monde qui les entoure, ainsi que de résolution des problèmes rencontrés.

- Encouragez les AJF à remettre en question la sagesse conventionnelle. Demandez-leur de réfléchir à leurs croyances et aux normes de leur communauté. Invitez-les à explorer, respectueusement, d'autres opinions.

- Commencez chaque échange par une question ouverte. Appliquez la méthode de la maïeutique, en posant la question " pourquoi ? " pour aider vos AJF à explorer leurs contradictions et à découvrir de plus profondes vérités.

- Encouragez la créativité. 
- Encouragez les AJF à prendre des risques dans leur réflexion et à ne pas craindre l'erreur. Soyez prête à démontrer vous-même cette approche.

- Organisez l'ES en petits groupes d'exploration thématique et d'activité collective.

- Encouragez l'analyse des problèmes et l'apport de solutions possibles sous différents points de vue.

5. Encourager les AJF à appliquer ce qu'elles ont appris dans leur vie et dans leur communauté pour les aider, en définitive, à devenir des citoyennes actives et la force d'un changement positif.

- Sélectionnez une session pertinente et des méthodes d'enseignement qui invitent la réflexion critique. Invitez constamment vos AJF à mettre la matière en rapport avec leur propre vie et le monde dans lequel elles évoluent. Encouragez-les à considérer la session à la lumière des principes d'équité et de justice sociale.

- Proposez des occasions d'apprentissage qui impliquent une recherche et une action dans le domaine des services ou dans la communauté. Veillez cependant à considérer la culture environnante et évitez de mettre vos AJF en danger.

- Considérez si et comment vous devrez défendre, au sein de votre établissement (et peutêtre aussi de votre communauté), la capacité et le droit des AJF de parler de leurs idées et de les mettre en pratique.

- Gardez à l'esprit que les AJF s'inspirent souvent de leurs enseignantes/mentores et des leaders de leur communauté.

L'utilisation de méthodes participatives actives présente plusieurs avantages pour l'AJF :

- Amélioration des compétences en matière de pensée critique

- Probabilité accrue que les participantes se souviennent et partagent de nouvelles informations

- Motivation accrue

- Amélioration des compétences interpersonnelles

À la page suivante, on vous présente des "astuces " clés pour une facilitation réussie par des mentores. 
1. Soyez passionnante !

Les participantes seront deux fois plus émotives que vous. Si vous êtes enthousiaste, les participantes seront deux fois plus enthousiastes. Si vous êtes ennuyeuses, les participantes seront deux fois plus ennuyées.

\section{Soyez une éléphante!}

Un éléphant a de grandes oreilles et une petite bouche : écoutez ce que disent les participantes, laissez-les parler. Un bon animateur parle $10 \%$ du temps. Posez des questions, faites rebondir les questions des participantes vers les participantes.

\section{Soyez neutre !}

N'imposez pas vos valeurs aux participantes. Le programme permet aux participantes de partager leurs valeurs librement sans l'influence des valeurs de la mentore.

\section{Soyez prête!}

Préparez-vous en expliquant les activités aux autres. Rassemblez des documents que vous aura besoin (soyez créatif si vous n'avez pas le matériel), et trouvez des réponses aux questions que vous n'avez pas pu répondre lors de la session précédente.

\section{Faites des contacts visuels !}

Le contact visuel de toutes les participantes signifie qu'elles sont à l'écoute. Si vous êtes à l'extérieur, assurez-vous que le soleil est dans le dos des participantes et dans vos yeux.

\section{Soyez honnête!}

Répondez honnêtement à toutes les questions. Ne soyez pas mal à l'aise si vous ne connaissez la réponse à une question. Dites simplement que vous ne la connaissez pas, mais que vous ferez tout votre possible pour la trouver.

\section{Soyez au même niveau que les participantes !}

Discussions sensibles : Si les participantes rient du mot "vagin", laissez-les en rire. Si elles sont autorisées à rire, elles seront plus enclines à en parler.

Langage : N'utilisez pas de grands mots qu'elles ne connaîtront pas. Traduisez si nécessaire pour que toutes les participantes comprennent ce dont vous parlez.
Soyez consciente de votre public et mettez-vous à leur place (travailler avec un groupe de AJF vulnérables).

8. Soyez cohérente!

II est facile d'enseigner aux meilleures participantes. Assurez-vous d'obtenir la participation de tout le monde. Posez des questions faciles ou simples aux participantes silencieuses pour que tout le monde participe.

\section{Soyez sensible!}

Sachez comment traiter les questions sensibles, en particulier lors des discussions où les participantes s'ouvrent et partagent leur expérience personnelle.

\section{Soyez positive!}

Le fait de rester positive maintient les niveaux d'énergie et crée un bon environnement pour la critique constructive et l'apprentissage. Faites des critiques constructives aux participantes entre deux éloges/points positifs.

\section{Faites-vous entendre !}

Volume, volume, volume! Assurez-vous que votre voix est entendue par toutes les participantes

\section{Soyez opportunes!}

Le programme prévoit du temps pour chaque activité. Ainsi, si vous faites une activité de 2 heures qui devrait être réalisée en 20 minutes, vos participantes s'ennuieront et vous manquerez d'autres activités importantes.

\section{Maîtrisez la situation!}

Les participantes s'amuseront tellement qu'elles oublieront peut-être qu'elles apprennent. Les stimulateurs rapides aident à contrôler les participantes afin que chacune fasse attention à ce que vous dites. Souvenez-vous des autres "astuces" (en particulier 1, 5, 7, 8, 10, 11, 12) pour être en contrôle de manière efficace.

\section{Soyez sur la bonne voie!}

Ne laissez pas les commentaires et les questions hors sujet des participantes vous détourner de votre objectif, qui est de transmettre les messages clés de l'activité.

45 Adapté de «Valoriser le potentiel des mentores: Recrutement, formation, et soutien des mentores pour des programmes dédiés aux adolescentes" du Population Council https://www.popcouncil.org/uploads/pdfs/2019PGY_MentorToolkit_fr.pdf 


\section{DES NORMES MINIMALES POUR ÉTABLIR LES LIENS AVEC D'AUTRES RESSOURCES COMMUNAUTAIRES QUI RÉPONDENT AUX BESOINS DES AJF}

\section{Les espaces sûrs servent de roues motrices pour relier les AJF aux services essentiels dans leurs communautés}

Il y a une limite à ce qui peut être accompli dans un espace sûr sous la direction de la mentore. Les mentores ne peuvent pas fournir, et il ne faut pas s'attendre à ce qu'elles fournissent à l'AJF, toutes les informations et tous les services dont elle pourrait avoir besoin. Au contraire, les mentores devraient être équipées pour être le pivot central d'une roue afin de coordonner l'accès aux ressources communautaires. Tous les adolescents d'une communauté n'ont pas le même accès aux ressources communautaires ; souvent, les plus pauvres, les plus vulnérables sont exclus par inadvertance (Figure 8); la mentore devrait pouvoir faciliter dans son espace sûr l'accès aux ressources communautaire pour les AJF participantes dans son espace sûr.

Figure 8. Représentation visuelle de la façon dont les adolescentes n'ont pas le même accès aux ressources communautaires (les AJF les plus pauvres sont souvent exclues)

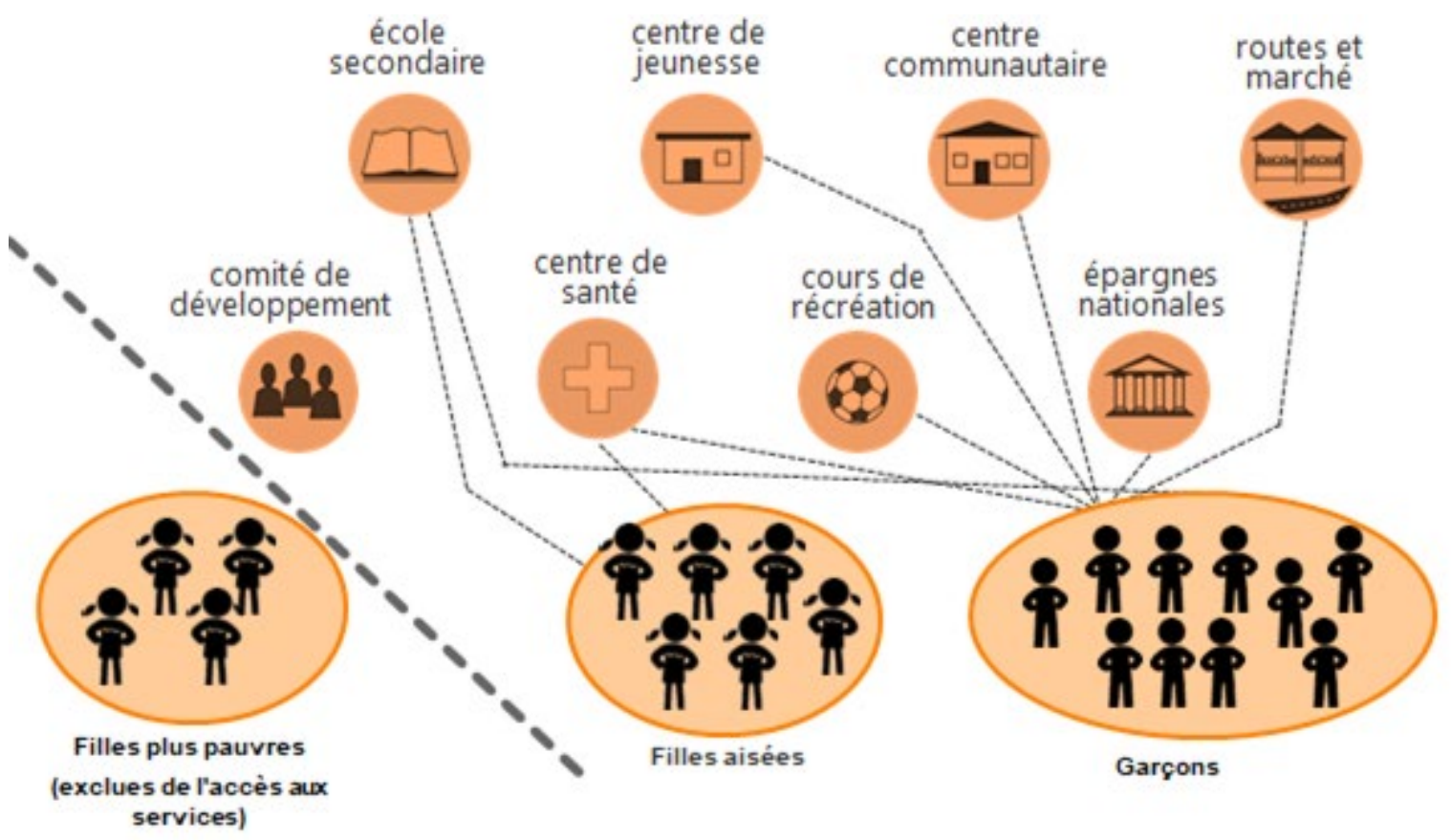

Il existe de nombreuses raisons pour lesquelles les ressources communautaires peuvent être inaccessibles à l'AJF. 
Tableau 7 : Des raisons possibles pour lesquelles les ressources communautaires peuvent être inaccessibles à l'AJF.

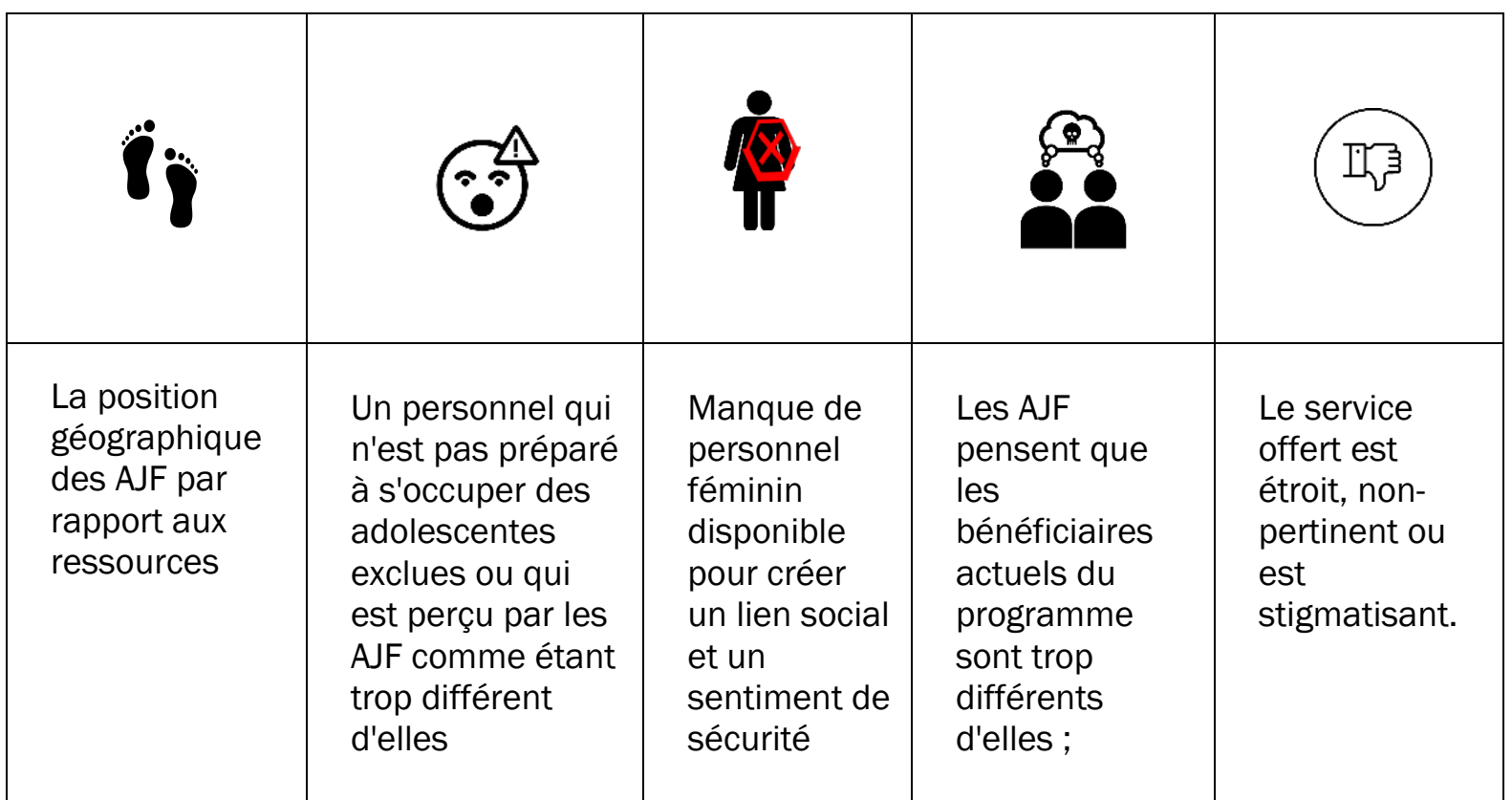

Pour aider à mettre les AJF en contact avec les services, il est utile d'effectuer un inventaire des installations, des services et des ressources dans la communauté accessible à pied grâce à un outil comme le balayage des ressources communautaires (décrit en détail à la page 17). Les résultats de l'exercice du balayage des ressources communautaires et du Girl RosterTM (décrit en détail à la page 10) peuvent être utilisés en tandem par les organisations de manière à planifier un meilleur accès des AJF aux ressources clés de la communauté et combler les lacunes éventuelles en matière de ressources. ${ }^{46}$

En vue d'accroître l'accès des AJF aux ressources communautaires de manière réfléchie, le personnel du programme doit tenir compte des limites spécifiques de la communauté du programme à couvrir. II peut être utile d'établir un engagement communautaire général pour atteindre les AJF appropriées. Cet engagement peut inclure des contrats formels par lesquels les dirigeants de la communauté acceptent publiquement de fournir un espace et un soutien aux AJF et à leurs mentores. Le processus d'apprentissage et l'engagement de la communauté aident à convertir les droits et politiques nationaux de base en ressources sur le terrain où " les vraies AJF obtiennent de vraies choses dans de vrais endroits".

L'obtention de services de santé n'est pas toujours une tâche aisée pour les AJF. Le fait même de demander des services représente une reconnaissance consciente 1) de leur activité sexuelle, 2) d'un risque de problème de santé/IST et/ou 3) d'une présence symptomatique.

II s'agit souvent là d'une importante étape mentale à franchir pour les AJF. Les autres obstacles concernent les coûts (du transport à la clinique et des services en soi), la peur d'être montrée du 46 Adapté de "Intentional design: reaching the most excluded girls in the, poorest communities: A Guide for
Practitioners and Advocates" du Population Council 
doigt et mal traitée par le personnel de la clinique, ou celle d'être vue dans le voisinage de la clinique et d'être ainsi couverte d'opprobres.

Vous pouvez établir le lien avec des services de santé conviviaux aux AJF, auxquels les participantes peuvent accéder, et envisager

l'accompagnement initial des AJF dans le cadre d'une « sortie éducative $\gg$.

Adapté de "Conception d'un programme centré sur les AJF: Boîte à outils pour l'élaboration, le renforcement et l'expansion de programmes
À moins que votre programme n'offre lui-même des services de santé, il vous appartient d'établir le contact avec des établissements de santé à proximité au cas où vous êtes tenue d'y envoyer les bénéficiaires de votre programme. Peut-être vous faudra-t-il aussi assurer le transport des AJF et obtenir de la clinique des tarifs spéciaux ou même la gratuité des services pour vos AJF. Offrir vos services à la clinique pour l'aider à se montrer plus sensible et " conviviale " aux AJF pourrait survenir. Une stratégie bénéfique à certaines AJF

consiste à les emmener en " sortie éducative " dans une clinique locale (prévenue de votre arrivée !). II se pourrait que les AJF aient eu un mauvais souvenir d'une expérience médicale passée. Les accompagner lors de cette première visite et les aider à établir le contact avec le secteur des services de santé peuvent être avantageux à leur recours ultérieur à ces services. Et par-dessus tout ne manquez pas d'inclure des informations quant aux modalités d'accéder à ces services dans le curriculum des espaces sûrs. ${ }^{47}$

En outre, avoir des visiteurs expertes invitées à participer aux sessions de groupe, peut être très bénéfique pour les AJF, particulièrement lorsque certains sujets et sessions tirent avantage de l'intervention d'une invitée ayant une expérience et une expertise sur le sujet. Cela s'applique en particulier aux sujets sur lesquels la mentore ne se sent pas en confiance, ou à ceux qui nécessitent des conseils techniques ou professionnels.

Afin de garantir le succès de la session, une mentore est tenue d'informer l'invitée à l'avance, lui fournir des guides de session qu'elle est censée animer et s'assurer que tous les documents pertinents sont prêts. De même, il est impératif de sensibiliser l'oratrice invitée sur les principes et les attitudes mentionnés tout au long du programme afin d'éviter de créer des contradictions. II est requis d'Informer au préalable les visiteurs expertes invitées que leur participation est volontaire et non rémunérées. 48

\footnotetext{
47 Adapté de "Conception d'un programme centré sur les AJF : Boîte à outils pour l'élaboration, le renforcement et l'expansion de programmes destinés aux adolescentes " du Population Council 48 Adapté de "Valoriser le potentiel des mentores: Recrutement, formation, et soutien des mentores pour des programmes dédiés aux adolescentes" du Population Council https://www.popcouncil.org/uploads/pdfs/2019PGY_MentorToolkit_fr.pdf
} 
La mise en place d'un système d'orientation solide en cas de VBG demande aux mentores de savoir quelles ressources sont offertes dans les environs. Lors de la formation initiale des mentores (voir page 31), ceux-ci peuvent dresser une carte des services de soutien disponibles dans leur communauté, tels que les services de santé sexuelle et reproductive du centre de santé locale, le soutien psychosocial, les unités de soutien aux victimes de la police et les systèmes d'orientation en matière de violences basées sur le genre (VBG). Cependant, tout le travail ne doit pas reposer seul sur la mentore, car dans les situations difficiles (comme les conflits avec les parents ou les employeurs), elles peuvent avoir besoin de

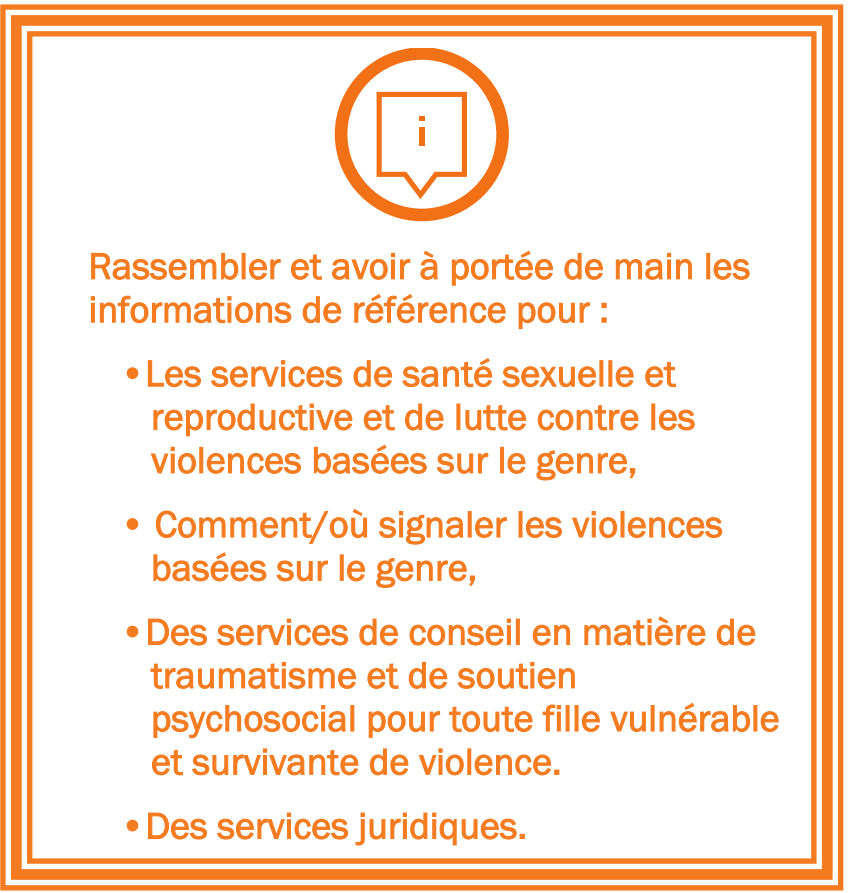
l'intervention du personnel du programme. 49

Qui plus est, la participation des AJF à l'espace sûr devrait les aider à développer une connaissance des signes et dangers des VBG, une capacité de réaction et de réponse à la violence interfamiliale (avoir un plan d'urgence/plan de sécurité), une connaissance des personnes à qui s'adresser en cas de violences physiques ou sexuelles, et par-dessus tout la connaissance de leurs droits humains. 50

En outre, l'espace sûr doit être le lieu où les adolescentes apprennent que les abus sexuels/ rapports sexuels forcés ne se limitent pas aux rapports sexuels ou à la pénétration, mais qu'ils incluent les agressions, les attouchements, ainsi que le fait de demander aux AJF de toucher les organes sexuels ou non de l'acteur.

Chaque adolescente est en droit de savoir que son corps est une propriété privée et personnelle. Personne ne doit la toucher sans son autorisation. Cela est d'autant plus important que certains abus sexuels sont commis par des membres de la famille ou des connaissances. La plupart du temps, les jeunes enfants sont la cible d'un inceste (une jeune personne peut être forcée à toucher, embrasser ou sentir les organes sexuels, ou à avoir un rapport sexuel réel avec un parent). ${ }^{51}$

49 Adapté de "Valoriser le potentiel des mentores: Recrutement, formation, et soutien des mentores pour des programmes dédiés aux adolescentes" du Population Council https://www.popcouncil.org/uploads/pdfs/2019PGY MentorToolkit fr.pdf

50 Adapté de "la santé des Adolescentes Vulnérables : un Investissement Stratégique Doublement Rentable " du Population Council

51 Adapté de " AGI-K Health Life Curriculum Wajir " du Population Council 


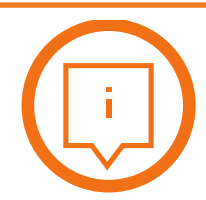

L'AJF doit savoir que les VBG sont ancrées dans un rapport de force inégal maintenu et perpétué par les normes sociales de genre, qui maintient la domination des hommes sur les femmes. Ainsi, en raison de sa position dans la famille, la personne plus âgée peut être en mesure de faire pression sur l'enfant pour qu'il accomplisse des actes sexuels sans avoir à recourir à la force. Ces crimes, y compris le viol, sont la faute de l'auteur ou de la personne âgée et non la faute de la victime ou de l'enfant.

Adapté de " AGI-K Health Life Curriculum Wajir " du Population Council

Les modules d'espace sûr doivent inclure une session à la fin de laquelle les participantes pourront :

- Identifier des adultes de confiance avec lesquels elles peuvent facilement s'ouvrir et partager leurs problèmes ;

- Identifier les ressources de leur école et de leur communauté qui aident les jeunes confrontés aux VBG et les lieux où elles peuvent signaler les cas de violence sexuelle

- Comprendre le rôle que les différentes ressources identifiées dans leur école et leur communauté peuvent jouer pour garantir l'accès à la justice des jeunes confrontés à la violence sexuelle ;

- Avoir la confiance nécessaire pour s'ouvrir et signaler les cas de violence sexuelle (en s'exerçant à signaler les cas de violence et d'abus sexuels) ;

- Comprendre ce qui fait d'une personne un adulte de confiance et qui est objectivement fiable.

À FAIRE : Former le personnel du programme et lui donner les compétences qui lui permettent de faire face aux VBG s'il s'agit d'un problème fréquent.

Renforcer le capital social et les plans de sécurité des AJF de manière à prévenir autant que possible les VBG.

Veiller à ce que I'AJF comprenne que les VBG est le résultat de problèmes structurels/sociétaux et non pas à cause de quelque chose qu'elle a fait ou de la façon dont elle s'est habillée. L'AJF n'est pas responsable des VBG ; ce n'est pas sa faute si cela lui est arrivé.

À ÉVITER : Attendre qu'il soit trop tard pour se préparer à faire face aux VBG ! 


\section{DES NORMES MINIMALES POUR MOTIVER ET MAINTENIR LA FRÉQUENTATION RÉGULIĖRE DES AJF DANS LES ESPACES SÛRS}

N'oubliez pas que votre programme ne doit pas 《 tout faire 》. II peut s'avérer utile d'élaborer un bon système d'orientation qui vous permet d'envoyer les AJF de votre programme vers un autre de la communauté avec lequel vous avez formé un partenariat.

Les programmes de formation professionnelle en sont un excellent exemple. II ne serait en effet pas nécessairement efficace pour votre programme d'organiser un cours de couture, même si de nombreuses AJF en expriment le désir. Une meilleure solution pourrait être de repérer les écoles de couture de votre région, d'établir une relation avec elles et d'y envoyer les AJF intéressées. ${ }^{52}$ (voir plus sur la formation professionnelle à la page 61)

\section{Amplifier les avantages du SWEDD en superposant les activités du SWEDD et en coordonnant les contenus}

La programmation croissante pour les adolescents est de nature multisectorielle. Les donateurs et les planificateurs de programmes reconnaissent que les déterminants sociaux comme le mariage précoce, les grossesses précoces et l'infection par le VIH se trouvent en dehors du secteur de la santé. Ces déterminants sociaux sont liés au statut socio-économique des AJF, à leur expérience scolaire, à la formation professionnelle et au travail, à leur mobilité, à leur situation géographique et à d'autres facteurs. De ce fait, les AJF ne connaissent pas seulement un type de risque, plutôt plusieurs qui intersectent. Par conséquent, celles qui sont plus exposées à un risque tel que le mariage précoce courent également d'autres risques, tel que le faible accès à l'école, faible mobilité dans la communauté, etc.

Dans la région du Sahel, ce sont généralement les mêmes AJF qui risquent l'abandon scolaire, le mariage précoce, les grossesses précoces et les possibilités limitées de génération de revenus (ou de moyens de subsistance), autant de circonstances qu'elles peuvent transmettre à leurs enfants, en particulier à leurs enfants adolescentes et jeunes filles.

Pour briser ce cycle, une approche multisectorielle qui s'attaque aux risques dans tous les secteurs et à différents niveaux : individuel, familial et communautaire est indispensable. Pour chaque AJF, le développement de ses compétences économiques - telles que l'éducation financière et l'accès à la micro-épargne - ainsi que de son soutien social et de ses compétences pratiques - telles que l'auto-efficacité pour accéder aux services de santé, la capacité de négociation - est plus susceptible de lui donner les moyens de faire face aux menaces et de réduire les risques que de s'attaquer seul à l'un de ces problèmes. "Les programmes à plusieurs composantes ont tendance à être plus performants que les programmes à une seule composante 53 ".

\footnotetext{
52 Adapté de "Conception d'un programme centré sur les AJF : Boîte à outils pour l'élaboration, le renforcement et l'expansion de programmes destinés aux adolescentes " du Population Council 53 Haberland, Nicole A., Katharine J. McCarthy, and Martha Brady. 2018. "Insights and Evidence Gaps in Girl-Centered Programming: A Systematic Review," GIRL Center Research Brief No. 3. New York: Population Council. https://www.popcouncil.org/uploads/pdfs/2018PGY_GIRLCenterResearchBrief_03.pdf
} 
Reconnaissant que les AJF vivent dans un contexte social, il est essentiel d'engager les frères, les maris, les parents et les autres membres de la communauté pour permettre aux AJF d'exercer leur pouvoir et d'amplifier les effets des programmes destinés aux individus.

Grâce à ses multiples sous-composantes et à la forte implication de plusieurs ministères sectoriels, le programme SWEDD est en bonne position pour s'attaquer aux risques dans tous les secteurs et à différents niveaux. La superposition ${ }^{54}$ d'activités connexes menées dans le cadre des différentes sous-composantes au sein des mêmes communautés, ancrées dans des espaces sûrs bien soutenus pour les AJF, permettrait d'accroître les avantages et de gagner en efficacité.

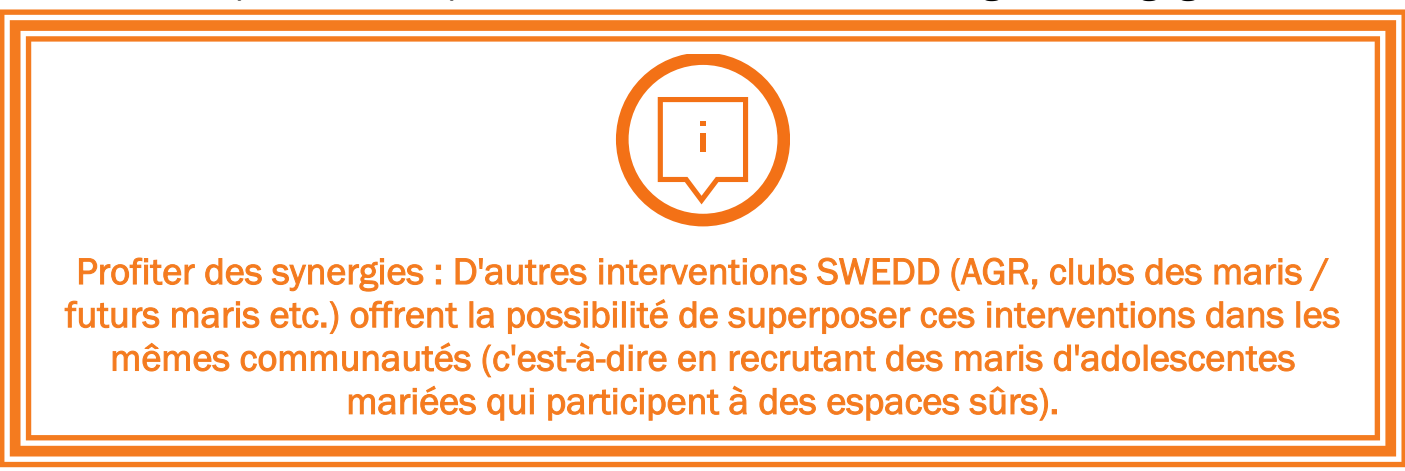

Le large portfolio de SWEDD crée une opportunité de coordination entre les différents partenaires et ministères d'exécution pour planifier les différentes interventions de SWEDD afin de s'appuyer les unes sur les autres.

Une autre façon de mettre en œuvre la superposition dans le cadre d'un grand projet tel que le SWEDD consiste à faire se chevaucher les interventions ciblant différentes démographies mais au sein de la même communauté ou du même réseau social. Pour le SWEDD, cela pourrait être la mise en œuvre délibérée de clubs des maris et de clubs des futurs maris où les participants sont les garçons et les hommes de la vie des filles qui participent aux espaces sûrs (leurs maris, pères et frères). Cette stratégie de superposition peut apporter des avantages supplémentaires aux filles elles-mêmes (puisque les hommes qui prennent les décisions dans leur vie traitent de sujets liés à l'égalité des genres). Toutefois, le fait de placer ces activités au sein d'une communauté peut également servir à "saturer", c'est-à-dire à faire bénéficier du projet une proportion plus dense de la population.

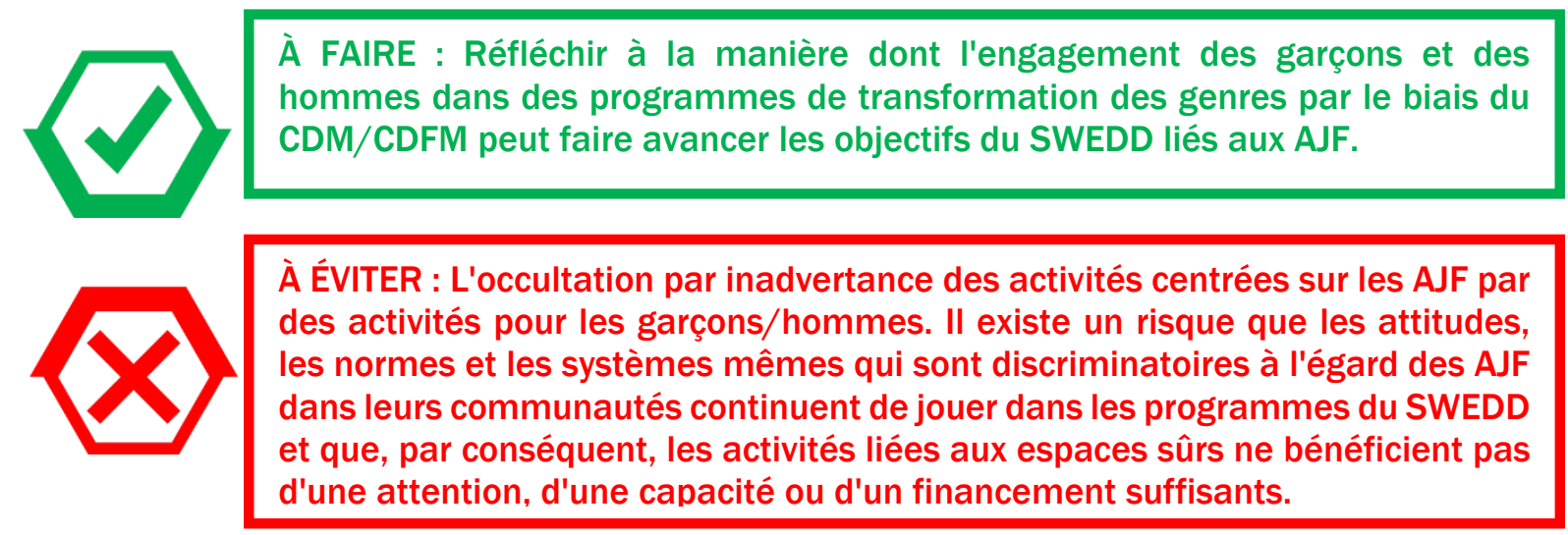

54 Cela signifie la mise en œuvre d'activités connexes au sein des mêmes communautés pour accroître les avantages et de gagner en efficacité. 
Compte tenu de son contenu multisectoriel, le SWEDD offre une excellente occasion d'investir dans l'autonomisation économique et le développement des compétences des AJF, qui sont parmi les moyens les plus urgents et les plus efficaces de faire progresser l'égalité des genres, l'éradication de la pauvreté et la croissance économique inclusive. À l'heure actuelle, les possibilités financières et de subsistance pour les AJF sont limitées dans les milieux où travaille SWEDD. Les AJF percevant un revenu travaillent souvent de longues heures dans des conditions parfois difficiles et risquées où elles peuvent être exploitées. En outre, le manque d'éducation financière des AJF signifie que les possibilités de générer des revenus ne se traduisent pas par des économies, ce qui réduit les ressources des jeunes femmes en cas de besoin.

Pour les mentores comme pour les AJF, apprendre à gérer l'argent et à se constituer un patrimoine financier peut contribuer à réduire leurs risques et à élargir leurs possibilités. Par exemple, la micro-épargne peut atténuer le risque qu'une AJF se tourne vers un homme plus âgé pour obtenir une aide financière en cas de crise. L'épargne peut également constituer un recours pour une AJF obligée quitter une situation où elle est confrontée à la violence d'un partenaire intime. L'éducation financière peut être utile dans le cadre d'un ensemble intégré de contenus autonomisant diffusés dans des espaces sûrs. L'éducation financière et la formation à des moyens de subsistance basés sur des compétences non techniques peuvent créer des aspirations et offrir une fenêtre d'opportunités pour accéder à l'enseignement professionnel ou à un emploi dans le futur. De plus, la possibilité d'acquérir des compétences, des réseaux et une expérience professionnelle pendant l'adolescence peut être un indicateur important de la capacité de production plus tard dans la vie.

En d'autres termes, les programmes conçus pourraient adresser l'influence des facteurs économiques sur la participation des AJF en créant des sources d'émancipation économique. L'émancipation économique directe (transfert d'argent par exemple) ou indirecte (à travers des formations sur l'éducation financière) des AJF peut inciter une participation régulière, réduire le coût de renonciation ainsi que l'opposition des parents ou des époux.

Pour déterminer le type d'activités d'autonomisation économique à inclure dans votre programme, il faut prendre en considération les facteurs suivants :

1. Les AJF avec lesquelles vous travaillez,

2. Les compétences de votre organisation,

3. Les partenariats ou liens possibles avec d'autres programmes, et

4. La préparation au marché.

Des recueils d'outils complets pourraient être compilés concernant chaque type de programme d'autonomisation économique (il en existe d'ailleurs déjà !) Nous allons donc nous limiter ici à un bref résumé des différents types de programmes et à quelques questions qui vous aideront dans vos décisions. Vous pourrez ainsi déterminer s'il vous serait utile d'introduire dans votre contenu des activités d'autonomisation économique, et lesquelles choisir.

En lisant le bref résumé des différentes options décrites ici, pensez à la liste de compétences et ressources que vous jugez importantes et appropriées pour les AJF de votre programme. Évaluez aussi honnêtement les capacités de votre organisation et votre préparation à ajouter un élément d'autonomisation économique à votre programme. Vous pourrez choisir ainsi la solution qui vous convient le mieux compte tenu de l'âge des AJF, de leur contexte et de leur expérience ou exposition antérieure à des activités d'autonomisation économique. 


\section{Éducation financière 55}

L'éducation financière est une compétence élémentaire de vie, qui se concentre sur les concepts de l'argent et la façon de bien le gérer. Elle couvre les connaissances, les compétences et les attitudes utiles à :

- L'adoption de bonnes pratiques de gestion de l'argent ;

- La prise de décisions relatives au gain d'argent, à l'épargne, à la dépense, à l'emprunt et à l'usage de services financiers ; et

- La fixation d'objectifs financiers, la budgétisation, le suivi de ses dépenses et l'épargne pour l'avenir.

Les AJF ont besoin d'une éducation financière pour acquérir une culture financière adaptée à leur âge et à leur vie. A titre d'exemple, la compréhension des concepts de base, comme la manière de différencier entre un " désir " et un " besoin ", et la manière d'établir un plan financier, sont des atouts importants pour toutes les AJF. L'éducation financière pourrait être envisagée pour l'intégration dans des structures d'espace sûr.

Au niveau de base, l'éducation financière se concentre sur les concepts de l'argent et les moyens de bien le gérer. Elle encourage l'épargne régulière, les dépenses judicieuses et les moyens de tirer le meilleur parti des ressources. Ces compétences servent de base aux AJF qui passent d'un rôle de dépendance à un rôle indépendant en matière de responsabilités financières et de prise de décision. De nombreux AJF, en particulier les adolescentes plus âgées, passent de la dépendance à l'égard de la famille pour gagner leur propre argent à la prise de décisions financières indépendantes. À un niveau très élémentaire, elles doivent savoir comment se débrouiller, saisir les opportunités générant des revenus, payer leurs dépenses et planifier leur avenir. L'éducation financière les aidera à apprendre à gérer leurs dépenses quotidiennes ainsi que l'avenir.

Comme pour les autres compétences, il est important de planifier les activités d'éducation financière qui profiteront le plus directement à votre segment spécifique. Les besoins financiers évoluent au fur et à mesure que les AJF grandissent, se marient et ont des enfants.

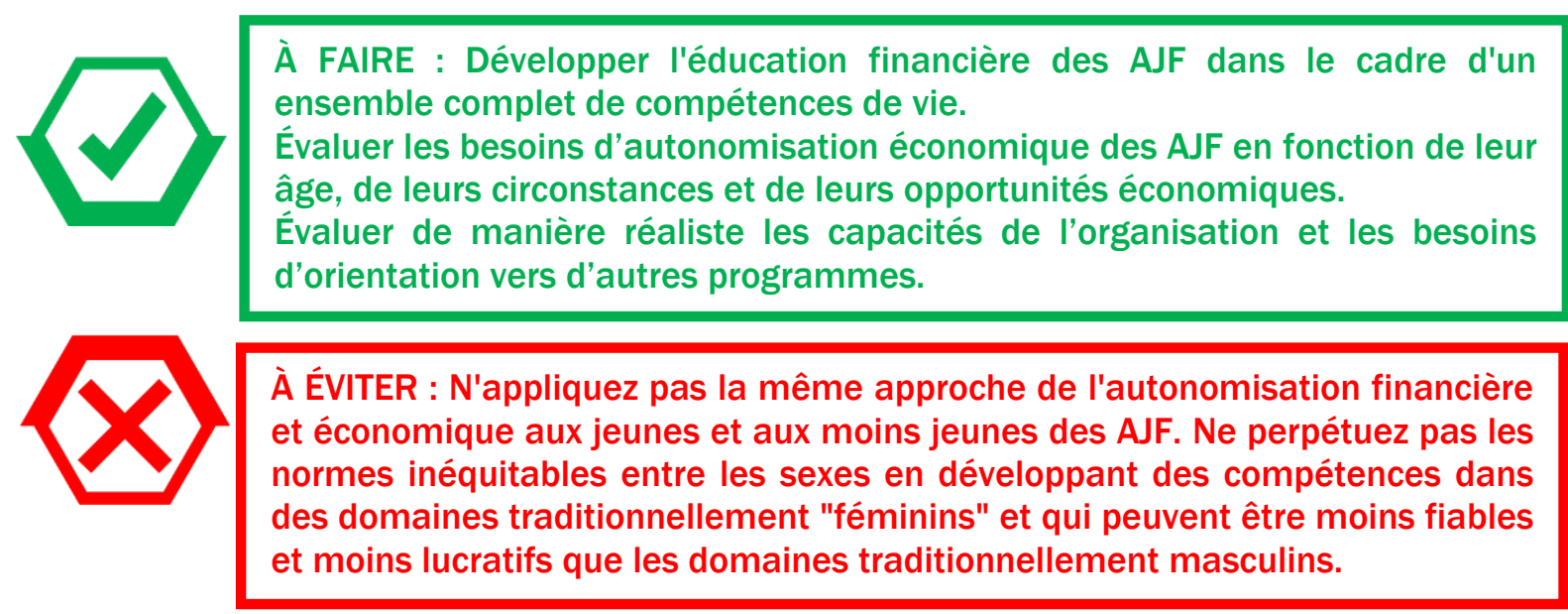

55 Adapté de "Conception d'un programme centré sur les AJF : Boîte à outils pour l'élaboration, le renforcement et l'expansion de programmes destinés aux adolescentes " du Population Council 


\section{Formation professionnelle et l'entrepreneuriat}

La finalité de l'espace sûr est de contribuer à l'autonomisation des AJF bénéficiaires. À cet effet, le programme conçoit un dispositif de formation professionnelle selon les aspirations des bénéficiaires en les encourageant à s'orienter vers les métiers non traditionnellement féminins. Le Projet SWEDD négocie un plan de formation de six à neuf mois. Ces cours de formation professionnelle peuvent être organisés les après-midis.

En outre, pour celles plus portées vers les activités de services ou de commerce, le Projet SWEDD les accompagne sous le format d'activités génératrices de revenus (AGR). Dans chacun des cas, le projet s'assure que soient dispensés deux modules : l'entrepreneuriat et le leadership, et le développement d'un business plan.

La formation professionnelle apporte aux AJF une qualification dans un certain domaine d'emploi : l'informatique, la conception graphique Web, la couture, la charpenterie, le conseil, la coiffure, la mécanique automobile, etc. La plus grande gageure des programmes de formation professionnelle concerne le risque de saturation du marché et celui des normes de genre. En premier lieu, les programmes forment souvent les AJF sans s'assurer auparavant de l'existence de débouchés sur le marché.

\section{II n'est pas rare de voir un programme former 30 jeunes coiffeuses à la fois, sans avoir toutefois répondu à la question de savoir s'il y aura 30 possibilités d'emploi pour les jeunes diplômées dans la communauté. II importe de bien évaluer la situation : plus que d'assurer la qualification des AJF, le but ultime étant de les aider à traduire cette qualification acquise en une activité génératrice de revenu.}

Deuxième gageure, les normes de genre tendent à imposer une adhérence stricte aux qualifications 《 acceptables 》 pour les AJF : la couture, la coiffure, la restauration, etc. Le premier problème, déjà mentionné, est le risque de saturation du marché. Ensuite, il pourrait être bénéfique aux AJF de recevoir une formation dans les domaines généralement réservés aux garçons et qui offrent souvent plus d'opportunités d'emploi et de meilleurs salaires. La prudence s'impose toutefois, car les AJF devront être soutenues et encadrées : il ne faudrait surtout pas les mettre dans une position qui les expose à un risque d'exploitation!

La formation à l'entrepreneuriat peut aider les AJF à développer les compétences nécessaires à l'établissement et à l'exploitation d'une entreprise couronnée de succès. Les AJF apprennent notamment à élaborer un plan d'entreprise, à décider du type d'entreprise le plus prometteur, à établir le prix de leur produit, à gérer les recettes et les dépenses de l'entreprise et à commercialiser leur produit.

Élément critique d'un bon programme d'entreprenariat, il importe d'aider les AJF à déterminer si elles désirent bel et bien assumer la responsabilité d'une entreprise. Toutes n'ont pas nécessairement la personnalité ou le désir de devenir propriétaires d'entreprise. Les AJF doivent comprendre les avantages et les inconvénients associés au lancement d'une entreprise, et elles doivent prendre leur décision en connaissance de cause. On a souvent tendance à croire, et cela peut être une erreur, que tout le monde désire lancer une entreprise et qu'il s'agit-là de la seule possibilité de génération de revenus.

L'inclusion de composantes d'autonomisation financière et économique dans la programmation des espaces sûrs peut contribuer à motiver la participation des AJF. Une tactique qui a réussi à retenir les participants consiste à intégrer des activités d'autonomisation économique dans des espaces sûrs. En superposant des activités génératrices de revenus avec des espaces sûrs, vous 
connecterez les AJF des espaces sûrs communautaires aux activités génératrices de revenus afin de réduire les abandons liés à la recherche de revenus ou d'emploi.

En agissant ainsi, les AJF mettront en pratique, grâce aux opportunités d'AGR que vous leur avez fournies, ce qu'elles ont appris lors des rencontres des espaces sûrs dans les modules d'alphabétisation financière. Cela peut être impressionnant pour l'ensemble du programme, car leur participation peut être un moyen pour elles de générer des revenus, ce qui à lui seul peut promouvoir le SWEDD dans les communautés et renforcer leur soutien.

\section{Stratégies efficaces pour encourager la fréquentation et maintenir la participation des 'AJF à des sessions dans un espace sûr}

Dans le but de maximiser la participation et retenir les AJF, le contenu du programme doit être pertinent pour elles. II est impératif pour elles de sentir et savoir que cela apporte quelque chose à leur vie et s'aligne à leurs besoins et aspirations ainsi que sur leurs expériences vécues, spécialement pour les AJF vulnérables qui courent les plus grands risques.

II se pourrait, qu'un programme éprouve ensuite des difficultés à retenir les AJF et à assurer leur participation régulière, même après un effort de recrutement couronné de succès. Les taux d'abandon peuvent être élevés, et la fréquentation irrégulière, notamment pour les raisons suivantes :

- Problème de jour/heure des rencontres (conflit avec les obligations scolaires et autres responsabilités).

- Durée du programme ou réunions trop longues, ou fréquence excessive (ou insuffisante) des rencontres.

- Manque d'appui des parents ou autres adultes importants (voir le chapitre 6).

- Besoin de garde des enfants ou des jeunes frères et sœurs dont les AJF sont responsables.

- Besoins des AJF non satisfaits/ennui.

Les programmes conçus en fonction spécifique des besoins des AJF tendent à minimiser ces problèmes. Si vous constatez cependant une baisse de présence et de participation, le moment est sans doute venu de procéder à une évaluation des besoins. (Voir l'étape 3 sur le SEA, à la page 68).

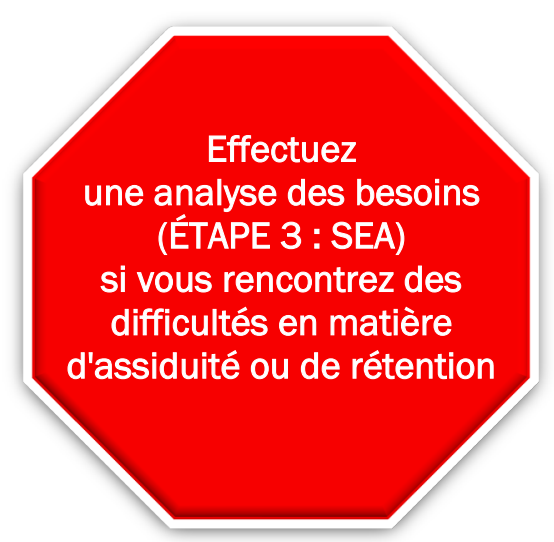


Pour la rétention des participantes, veillez à ce que la participation à un espace sûr ne crée pas de conflit entre les activités habituelles des AJF et leur programme. Cela consiste, pour les espaces sûrs scolaires, à ce que les responsables de la mise en œuvre s'assurent que le programme n'entrave pas les travaux scolaires des participantes. Quant aux AJF dans les communautés, s'assurer que la participation s'aligne avec leurs responsabilités au ménage, au marché ou aux champs et ne les entrave pas.

Une autre tactique de rétention tout aussi importante est l'emplacement, le lieu de rencontre des espaces sûrs, qui doit être facilement accessible, sûr et n'incombe pas de frais de transport pour les AJF. Le lieu choisi doit garantir l'intimité des AJF et leur offrir un espace où elles se sentent à l'aise pour parler et partager leurs opinions.

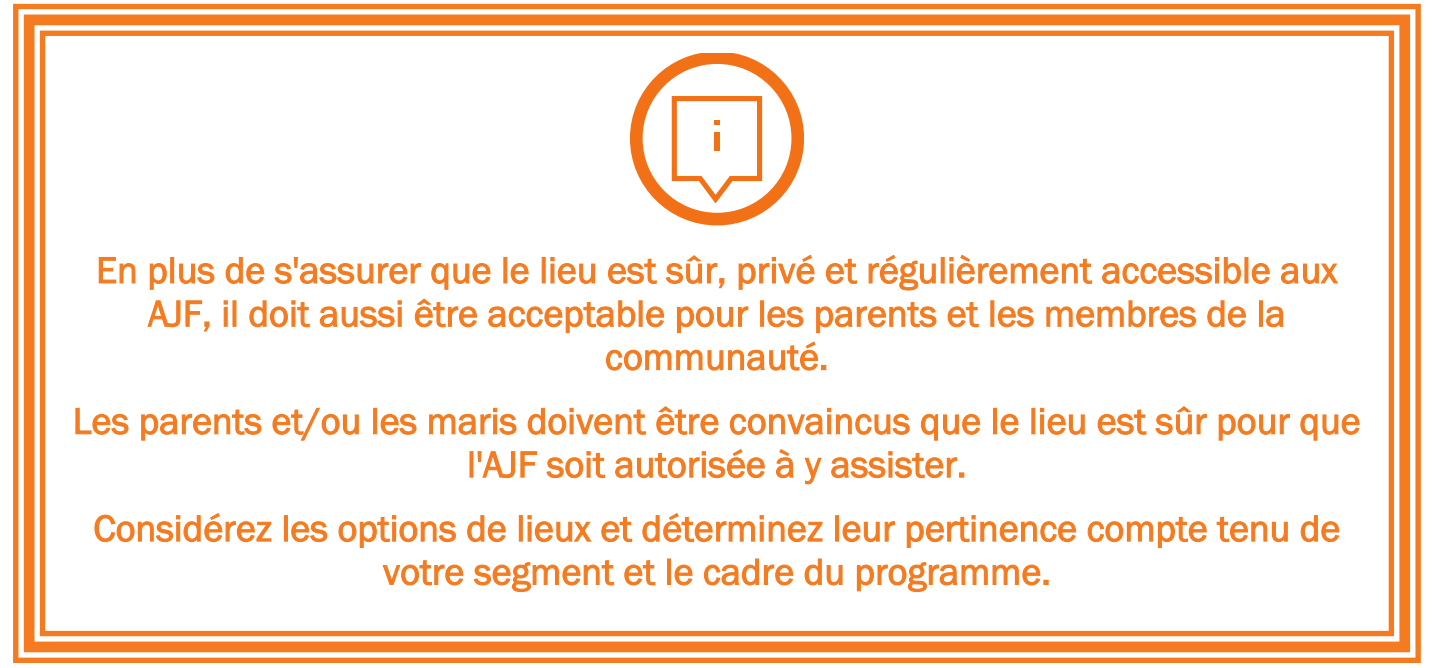

En outre, la provision d'une motivation, telle que des collations, peut être un moyen d'encourager et de motiver la participation. Cette motivation doit être planifiée et mise en œuvre systématiquement dans les espaces sûrs SWEDD. Les dispositions logistiques pour la fourniture de collations devraient dès lors faire partie de la planification et ne devraient pas surcharger les superviseurs ou les distraire de leurs fonctions essentielles.

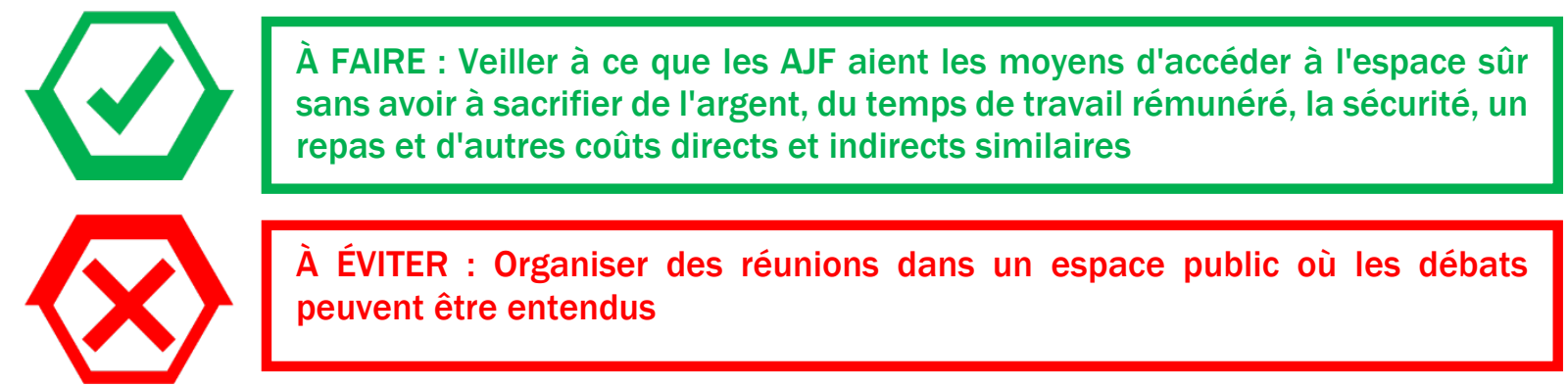




\section{DES NORMES MINIMALES POUR ADAPTER LA PROGRAMMATION DES ESPACES SÛRS À LA COVID-19 ET À D'AUTRES CRISES}

Les crises telles que le virus Ebola, la famine et les troubles politiques perturbent les activités au niveau communautaire. Avec l'émergence du coronavirus, et la pandémie mondiale de la COVID19, le monde a dû s'adapter à une nouvelle crise qui a perturbé les activités normales dans tous les secteurs.

"Les adolescentes font partie des groupes censés bénéficier des mesures que les gouvernements adoptent pour contrôler la COVID-19, telles que les restrictions de déplacements et les fermetures d'entreprises et d'écoles. Toutefois, étant donné le double désavantage auquel les adolescentes sont confrontées en raison de leur âge et de leur sexe, même les approches qui réduisent efficacement la menace de transmission de la COVID-19 peuvent exacerber d'autres menaces pour leur santé, leur sécurité et leur bienêtre. " 56

La COVID-19 présente les menaces directes (infection) et indirectes (réponse COVID-19) dans tous les secteurs et toute adaptation doit protéger les AJF, les mentores, les superviseurs, les familles et les communautés contre ces deux menaces. Les effets les plus néfastes seront les plus ressentis là où des inégalités existent déjà.

- Les pires effets de la COVID-19 le long des lignes de failles connues mettent en évidence les inégalités et la vulnérabilité existantes en raison du sexe, de l'âge et d'autres facteurs.

- La COVID-19 crée et renforce l'exclusion et l'isolement social : un défi prioritaire pour les adolescents.

Avant de créer un plan d'adaptation de la mise en œuvre des espaces sûrs, il convient de comprendre les implications pour les adolescentes dans les principaux résultats : santé, éducation, moyens de subsistance, etc. et prendre en compte les opportunités spécifiques aux adolescentes et les opportunités à l'échelle de la population, y compris les AJF.

Nous savons qu'en temps de crise, comme celle de la COVID-19, la charge domestique de l'AJF est accrue.

\section{"En règle générale, l'expérience des épidémies a montré que la charge domestique des femmes* s'alourdit avec, rendant leur part de responsabilités domestiques encore plus élevée.... "}

UNFPA, COVID-19 A Gender Lens, 2020

* et des AJF'!

\footnotetext{
56 Blake, Sarah C.; Temin, Miriam, 2020, "Evidence review: Promoting adolescent girls' health and wellbeing in low-resource settings in the era of COVID-19", https://doi.org/10.7910/DVN/B3FZ60, Harvard Dataverse, V1
} 
Les premiers travaux ${ }^{57}$ sur les effets de la COVID-19 sur les adolescentes ont identifié sept voies de risque, dont chacune s'accompagne d'opportunités :

1. La santé

2. L'éducation

3. Les moyens de subsistance

4. La violence

5. L'eau et l'assainissement

6. Le transport

7. La technologie en matière de connectivité

Exemples d'adaptations utilisées dans les programmes avec des espaces sûrs face à la COVID-19 :

1. Modification de l'organisation/structure de l'espace sûr
a. Se réunir à l'extérieur
b. Se réunir en petits groupes
c. Répartir les participants (ne pas s'asseoir trop près les unes des autres)
d. Porter un masque
e. Éviter les activités qui reposent sur le contact physique ou la proximité

2. Envisager d'autres méthodes d'engagement (si les réunions en personne ne sont pas possibles)

a. Engager une discussion au téléphone avec les adolescentes du groupe de bénéficiaires du programme, reconnaître que toutes les filles, en particulier les plus marginalisées, n'ont pas accès à un téléphone.

b. Essayez d'adapter des espaces sûrs pour diffuser le contenu : par exemple, l'apprentissage par radio.

3. Autres moyens de s'engager et de soutenir les communautés où vous travaillez

a. Avec les participants, faciliter les activités génératrices de revenus qui peuvent également profiter à la communauté, comme la fabrication de savon ou la couture de masques.

b. Travailler avec les communautés pour se préparer et s'adapter à la règlementation sécuritaire COVID-19.

c. Diffusion de conseils en matière de prévention des infections, de santé et d'hygiène.

57 Plan d'investissement pour les Adolescentes (AGIP). Plan-International, Population Council, Girls not Brides, ICRW, ODI, autres 
Étant donné la nature changeante des crises, les responsables de la mise en œuvre apprennent constamment de nouvelles façons d'adapter les programmes et d'atteindre les participants au programme en leur sein. Pour en savoir plus sur les adaptations spécifiques à ce jour (novembre 2020), cliquez ici ou visitez le lien dans la note de bas de page ci-dessous ${ }^{58}$ et consultez régulièrement les mises à jour. (NOTE : disponible uniquement en anglais, malheureusement)

\section{Reconnaissez l'opportunité de reconstruire en mieux!}

Comment les enseignements tirés de la pandémie COVID-19 peuvent-ils éclairer la manière dont les pays fonctionneront à l'avenir ? Comme dans l'exemple du Population Council Kenya d'une "école sans murs", c'est une occasion de contourner certaines des barrières traditionnelles à l'éducation. Si nous changeons notre façon de penser à l'endroit et à la manière dont l'éducation et le contenu de l'espace sûr sont dispensés, nous pourrions potentiellement atteindre plus d'AJF.

58 https://buildcommunity4girls.org/fieldexperience-covid-response-introduction/ 


\section{ÉTAPE 3 : SUIVI, ÉVALUATION ET APPRENTISSAGE (SEA)}




\section{ÉTAPE 3 : SUIVI, ÉVALUATION ET APPRENTISSAGE (SEA)}

Dans ce chapitre, nous allons décrire l'objectif de chaque élément d'un plan de suivi, d'évaluation et d'apprentissage (SEA), qui se doit d'être informatif, utilisable et réussi. Les sections ci-dessous en donneront les explications :

- Le suivi, l'évaluation et l'apprentissage, qu'est-ce que c'est ?

- Chaque composante est distincte, mais complète les autres pour informer les programmes.

- Des normes minimales pour planifier et exécuter des plans de suivi utiles et réalisables.

- Des normes minimales sur ce qu'il faut mesurer pour évaluer votre succès.

- Des normes minimales pour l'établissement d'un système et d'une culture d'apprentissage.

Un plan de SEA approprié peut vous aider à vérifier que votre programme d'espaces sûrs réalise les activités prévues et les objectifs du programme. II vous aide également à mesurer le succès de votre programme en suivant le type de changement qui se produit dans la vie de vos groupes cibles prioritaires. Un plan SEA approprié doit aussi contribuer à améliorer le programme à chaque étape de sa mise en œuvre. Le SEA crée une boucle de rétroaction qui peut informer et améliorer la coordination, la conception et la mise en œuvre des espaces sûrs.

Le projet SWEDD a élaboré le " Manuel pour le suivi, la supervision et l'évaluation des Espaces Sûrs pour les AJF " détaillant le processus de S\&E des espaces sûrs. II présente quelques outils essentiels de S\&E à chaque étape du processus de suivi et d'évaluation : (1) planification du S\&E ; (2) évaluation initiale ; (3) activités de suivi et de supervision ; et (4) évaluation des résultats. Les outils sont fondés sur des expériences antérieures dans la mise en œuvre des espaces sûrs et d'autres initiatives communautaires, ainsi que sur les curricula développés dans le cadre du projet SWEDD.

Si les pays utilisent les mêmes outils en concordance, il sera possible d'agréger toutes les données SEA - ce qui serait instructif et bénéfique pour la SWEDD dans son ensemble ainsi que pour les efforts similaires en cours dans la région.
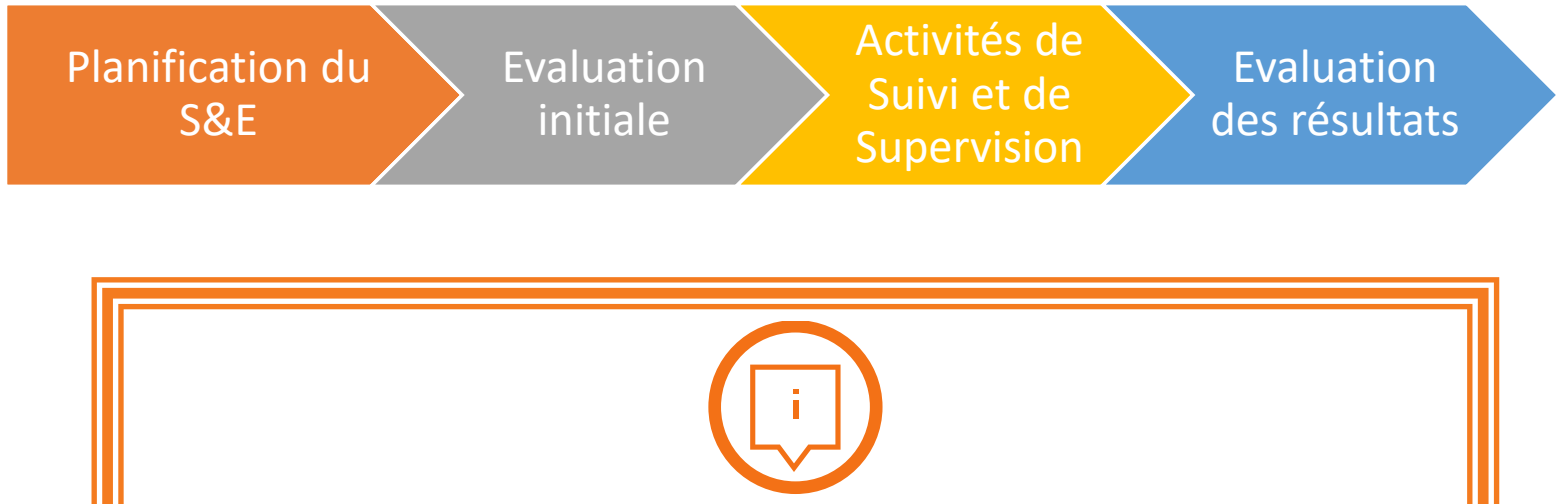

Bien qu'il soit essentiel et nécessaire d'adapter les plans SEA au contexte du pays et aux besoins du programme, il est important de discuter de vos plans SEA avec le point focal régional SEA pour assurer l'harmonisation à travers les pays SWEDD ; ce qui permettrait un échange et une comparaison plus transparente entre tous les pavs concernés. 
S'appuyant sur le manuel de S\&E, ce chapitre du guide des espaces sûrs fournit des conseils sur les normes minimales requises pour un plan SEA de chaque pays. II contient également des liens vers le manuel pour encourager l'utilisation d'outils harmonisés dans les pays. En outre, sur la base des enseignements tirés du processus SEA de la conférence SWEDD 1, ce chapitre explique comment procéder : (1) élaborer un plan garantissant que les données collectées sont utilisables ; (2) mobiliser des mentores, alphabétisées ou non, pour le suivi de routine ; (3) obtenir des données qualitatives pour l'amélioration du programme ; et (4) suivre les activités et les résultats au niveau communautaire et identifier leur lien avec votre programme d'espaces sûrs.

\section{Les distinctions entre le suivi, l'évaluation et l'apprentissage et comment une "culture de l'apprentissage " peut soutenir un SEA efficace}

Avant d'élaborer votre plan SEA, il est important de comprendre les différentes composantes et leur rôle dans la bonne mise en œuvre de votre programme. Chaque composante d'un plan SEA sert un objectif distinct, bien qu'elles soient toutes complémentaires. La figure 9 ci-dessous décrit l'objectif de chaque composante et la manière dont elles sont utilisées pour informer les programmes.

\section{Figure 9 : Les distinctions entre le suivi, l'évaluation et l'apprentissage}

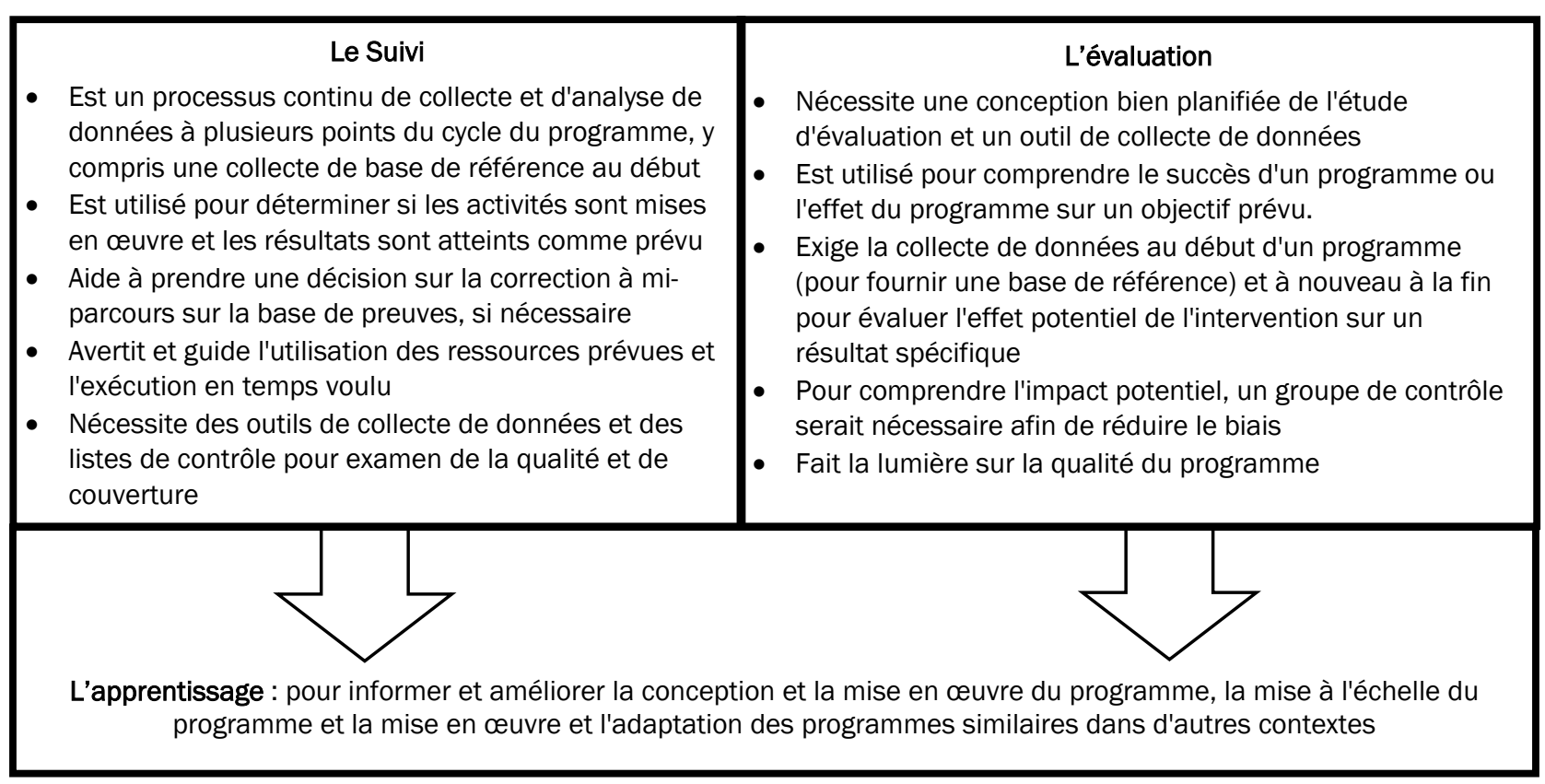

Un plan SEA approprié doit être "adapté à l'objectif" du programme, ce qui suppose qu'il ne peut pas être trop ambitieux ou complexe, sinon il échouera. Avant de développer un plan avec les mécanismes SEA que vous utiliserez, il est important de considérer ce que votre programme peut réaliser de manière réaliste ; quels étaient les objectifs de votre projet (par exemple : quelles compétences vouliez-vous que l'AJF acquière) ?

Il convient de déterminer la " taille correcte " du plan de SEA pour la réussite de votre programme, à savoir: assurez-vous de ne collecter que les données de S\&E qui sont adéquates à un apprentissage utile et utilisable ; il n'est pas nécessaire de collecter des données qui ne seront jamais analysées. Les données de suivi et d'évaluation devraient englober les principes suivants 
et être : crédibles, valides, fiables et utilisables. (Plus d'informations à ce sujet dans la section "S'assurer que les données sont crédibles, valides, fiables, et utilisables " à la page 84). 59

\section{DES NORMES MINIMALES POUR PLANIFIER ET EXÉCUTER DES PLANS DE SUIVI UTILES ET RÉALISABLES}

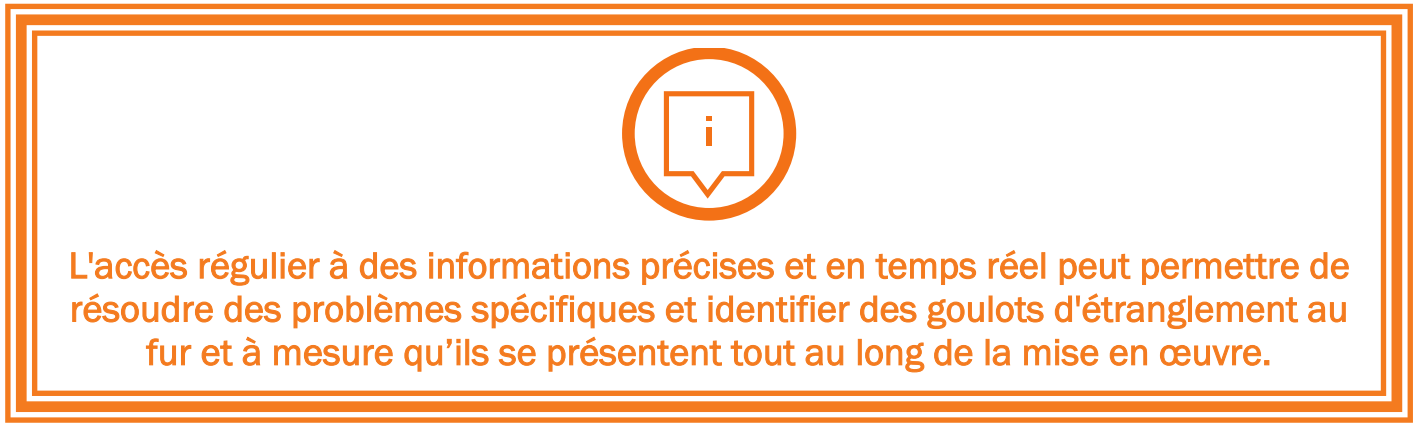

\section{Un but et un objectif clairement définis pour le suivi d'un espace sûr}

Le suivi consiste en une observation systématique et ciblée, et en une collecte de données en temps utile pour vérifier si les activités du programme sont mises en œuvre comme prévu suivant une certaine fréquence, un calendrier défini et de séquence préétablie au besoin. Plus précisément, le suivi suit et mesure les activités du programme en informant sur le nombre d'activités réalisées, le lieu, les personnes concernées, la période établie, etc. En outre, les données de suivi peuvent être un outil précieux pour suivre la faisabilité de la mise en œuvre de votre programme, identifier les problèmes potentiels à un stade précoce et aider les gestionnaires à suivre une correction en cours d'exécution afin d'atteindre l'objectif du programme.

- Les activités sont-elles mises en œuvre comme prévu ?

- Les activités répondent-elles à la cible visée?

- La qualité de la mise en œuvre est-elle satisfaisante?

- Les outils, le canal et l'équipement utilisés pour communiquer les messages sont-ils culturellement acceptables et efficaces ?60

59 Copyright 2016 Innovations for Poverty Action. Goldilocks: Finding the Right Fit in Monitoring \& Evaluation is made available under a Creative Commons Attribution-NonCommercial-NoDerivatives 4.0 International License. https://www.poverty-action.org/publication/goldilocks-finding-right-fit-monitoringevaluation

60 Population Council: "Training manual on basic monitoring and evaluation of social and behavior change communication health programs" https://www.popcouncil.org/uploads/pdfs/2014RH_BCCTrainingManual.pdf 


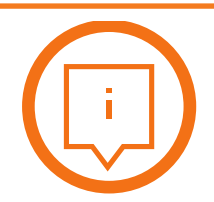

La première étape de la planification de votre plan de suivi est de vous référer à ce que votre projet vise à accomplir. Quels sont vos intrants et vos résultats attendus ? Partant, votre plan de suivi et d'évaluation doit identifier les indicateurs au niveau des intrants, des extrants (produits), et des réalisations (effets directs) dans la mesure où ils sont liés à la réalisation de l'objectif de votre programme.

N'oubliez pas, il ne faut pas essayer de faire plus que ce qui est possible ou utile. En fonction de l'objectif de votre programme, fixez des objectifs réalistes pour votre plan de suivi qui serviront de base à la mise en œuvre du programme.

À FAIRE : Élaborer et utiliser des outils de suivi qui recueillent des informations importantes pour votre programme

À ÉVITER : Rassemblez plus d'informations qu'il n'en faut

Définir quelles données de suivi vous devez collecter pour déterminer si vous progressez vers vos objectifs

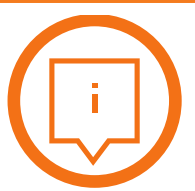

Avant de développer votre planification et vos outils de suivi et d'évaluation, il est important d'examiner des questions telles que :

- Qui va créer et utiliser les outils ; qui est chargé de vérifier s'ils sont terminés?

- À quel moment seront utilisés les outils?

- Qui est responsable de l'analyse et de la présentation des informations que les outils vont collecter?

- Combien de fois et sous quel format se feront l'analyse et la présentation?

Adapté de " Développer les compétences de protection des AJF : Ensemble d'Outils pour la Conception d'un Programme " du Population Council 
Le suivi du programme aide les gestionnaires à :

1. Évaluer la fidélité au modèle, ou le fait que le programme est mis en œuvre comme prévu,

2. Suivre les progrès et le rythme de la mise en œuvre et le comparer au plan,

3. Faire des ajustements à mi-parcours pour améliorer les résultats du programme en fonction d'un certain nombre de facteurs, notamment : la perception et les réactions des AJF, des nouveaux aspects que vous apprenez sur les AJF et que vous devez inclure, les défis rencontrés et les opportunités qui se présentent, en plus des modèles d'utilisation, y compris la sous-utilisation et la surutilisation.

Sur la base d'une boîte à outils développée par Innovations for Poverty Action ${ }^{61}$, il existe cinq types de données clés dont l'objectif est de collecter pour le suivi et la responsabilisation des programmes. Ils sont résumés dans le Tableau 8 ci-dessous.

61 Innovations for Poverty Action. 2016 "Monitoring for Learning and Accountability" https://www.povertyaction.org/sites/default/files/publications/Goldilocks-Toolkit-Monitoring-for-Learning-andAccountability_1.pdf 
Tableau 8: Cinq types de données clés pour le suivi et la responsabilisation des programmes

\begin{tabular}{|c|c|c|}
\hline Type de données & Comment les données sont utilisées & Les sources ou exemples d'outils \\
\hline $\begin{array}{l}\text { Données } \\
\text { financières }\end{array}$ & $\begin{array}{l}\text { Les données financières sur les coûts } \\
\text { peuvent aider les programmateurs } \\
\text { dans les domaines suivants: (1) } \\
\text { comprendre comment les ressources } \\
\text { sont utilisées pour les activités du } \\
\text { programme; (2) comprendre les coûts } \\
\text { réels du programme ; (3) identifier les } \\
\text { domaines qui nécessitent des } \\
\text { investissements; (4) informer à } \\
\text { l'échelle }\end{array}$ & $\begin{array}{l}\text { Rapports sur les dépenses des } \\
\text { projets } \\
\begin{array}{l}\text { Pièces justificatives des } \\
\text { dépenses effectuées par l'ONG }\end{array}\end{array}$ \\
\hline Suivi des activités & $\begin{array}{l}\text { Les données sur les activités clés et } \\
\text { les résultats qui sont détaillés dans } \\
\text { votre plan de suivi directement lié à } \\
\text { l'objectif de votre programme. Ces } \\
\text { données sont utiles pour comprendre } \\
\text { quelles activités sont à l'heure et dans } \\
\text { les délais prévus et les différences } \\
\text { selon le lieu afin de permettre aux } \\
\text { programmes de s'adapter en } \\
\text { conséquence. }\end{array}$ & $\begin{array}{l}\text { Dans le manuel SWEDD de S\&E } \\
\text { pour les espaces sûrs: } \\
\text { - Outil de suivi de la } \\
\text { fréquentation des espaces } \\
\text { sûrs /fiche de présence } \\
\text { mensuelle aux sessions. } \\
\text { - Canevas du rapport de suivi et } \\
\text { de supervision } \\
\text { - Outil pour la supervision des } \\
\text { mentores } \\
\text { - Liste de contrôle pour la } \\
\text { supervision des espaces sûrs } \\
\text { - Les fiches d'évaluation PRE- } \\
\text { TEST et POST-TEST } \\
\text { - Les fiches de suivi de } \\
\text { l'utilisation des services de } \\
\text { santé. }\end{array}$ \\
\hline Ciblage & 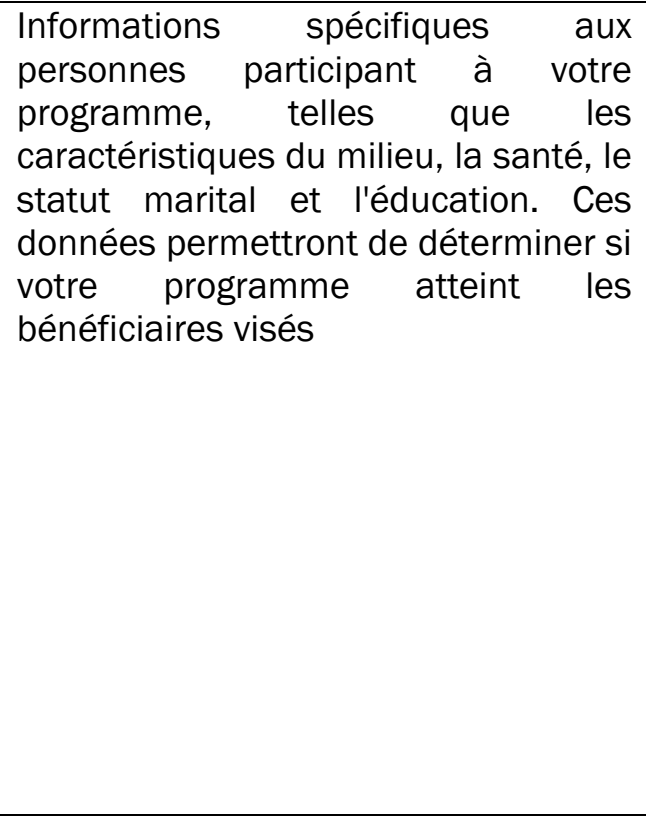 & $\begin{array}{l}\text { Dans le manuel SWEDD de S\&E } \\
\text { pour les espaces sûrs : } \\
\text { - Fiche de recensement des } \\
\text { ménages pour l'identification } \\
\text { des filles participantes } \\
\text { - Feuille de synthèse des } \\
\text { inscriptions à l'espace sûr } \\
\text { - L'exercice de couverture du } \\
\text { Population Council (qui se } \\
\text { trouve dans le document : } \\
\text { "Développer les compétences } \\
\text { de protection des filles: } \\
\text { Ensemble d'Outils pour la } \\
\text { Conception d'un Programme" } \\
\end{array}$ \\
\hline
\end{tabular}




\begin{tabular}{|c|c|c|}
\hline $\begin{array}{l}\text { Adoption et } \\
\text { Engagement }\end{array}$ & $\begin{array}{l}\text { Les données d'acceptation sur les } \\
\text { espaces sûrs impliqueraient } \\
\text { d'analyser le nombre de filles que } \\
\text { votre programme a essayé de recruter } \\
\text { par rapport au nombre de celles qui } \\
\text { ont effectivement participé au } \\
\text { programme. Les données } \\
\text { d'engagement impliqueraient } \\
\text { d'analyser le nombre de filles qui } \\
\text { continuent à participer à votre } \\
\text { programme une fois inscrites. } \\
\text { Ces deux types d'informations aident } \\
\text { le programme à comprendre s'il } \\
\text { répond aux besoins des bénéficiaires } \\
\text { et des communautés visées. }\end{array}$ & $\begin{array}{l}\text { Dans le manuel SWEDD de S\&E } \\
\text { pour les espaces sûrs: } \\
\text { - Fiche d'admission dans les } \\
\text { espaces } \\
\text { - Outil de suivi de la } \\
\text { fréquentation des espaces } \\
\text { sûrs /fiche de présence } \\
\text { mensuelle aux sessions. }\end{array}$ \\
\hline Feedback & $\begin{array}{l}\text { Fournit des informations sur les points } \\
\text { forts et les points faibles du } \\
\text { programme "Espaces sûrs" du point de } \\
\text { vue des participants et des mentores. }\end{array}$ & $\begin{array}{l}\text { Dans le manuel SWEDD de S\&E } \\
\text { pour les espaces surs: } \\
\text { - Questionnaire pour } \\
\text { l'évaluation de la } \\
\text { satisfaction des filles } \\
\text { - Évaluation en "H» pour } \\
\text { une appréciation globale de } \\
\text { la mise en œuvre des } \\
\text { espaces sûrs } \\
\text { - Feux tricolores pour } \\
\text { l'évaluation } \\
\text { des changements dans la } \\
\text { vie des filles }\end{array}$ \\
\hline
\end{tabular}

\section{Visites de sites des espaces sûrs:}

Une gestion efficace des programmes comprend un suivi fréquent au niveau de la communauté et de l'espace sûr lui-même. Des visites de sites d'espaces sûrs sont essentielles (mais pas suffisantes) pour permettre une compréhension approfondie du fonctionnement du programme et de la manière dont il peut être amélioré.

Les évaluations quantitatives et qualitatives peuvent être utilisées pour explorer les domaines suivants liés à votre espace sûr :

- $\quad$ La Structure: La "structure" de l'espace sûr fonctionne-t-elle pour les participants et la mentore ? (Lieu de la réunion, jour de la semaine, heure de la journée, heures, etc.)

- Le contenu: Quels sont les sujets bien accueillis ? Moins bien accueillis ? Quelles sont les activités de l'AJF ? Quels sont les atouts dont l'AJF est impatiente de prendre connaissance?

La performance de la mentore: Dans quelle mesure la mentore adhère-t-elle au curriculum ? Est-elle capable d'engager I'AJF et de mener une session dynamique?

Est-il injuste de rendre visite aux mentores alors qu'elles ne s'y attendent pas ? Le but d'une visite inopinée n'est pas de surprendre la mentore, mais d'évaluer ses performances lors d'une session typique, afin que les superviseurs puissent lui fournir des conseils de soutien basés sur ce qui va bien et sur la manière de s'améliorer. Si les mentores savent que le personnel du programme vient, elles peuvent se préparer plus que d'habitude, de sorte que la session ne serait pas le reflet fidèle de leur préparation habituelle et de leurs compétences d'animation. Le personnel du programme doit savoir si les mentores se préparent aux réunions et comment elles mènent leurs activités afin de fournir un retour d'information pour l'amélioration des performances. 


\section{Conseils sur l'identification des indicateurs de suivi}

Au cours du processus de planification du programme, les indicateurs devraient être définis et liés aux activités et aux objectifs.

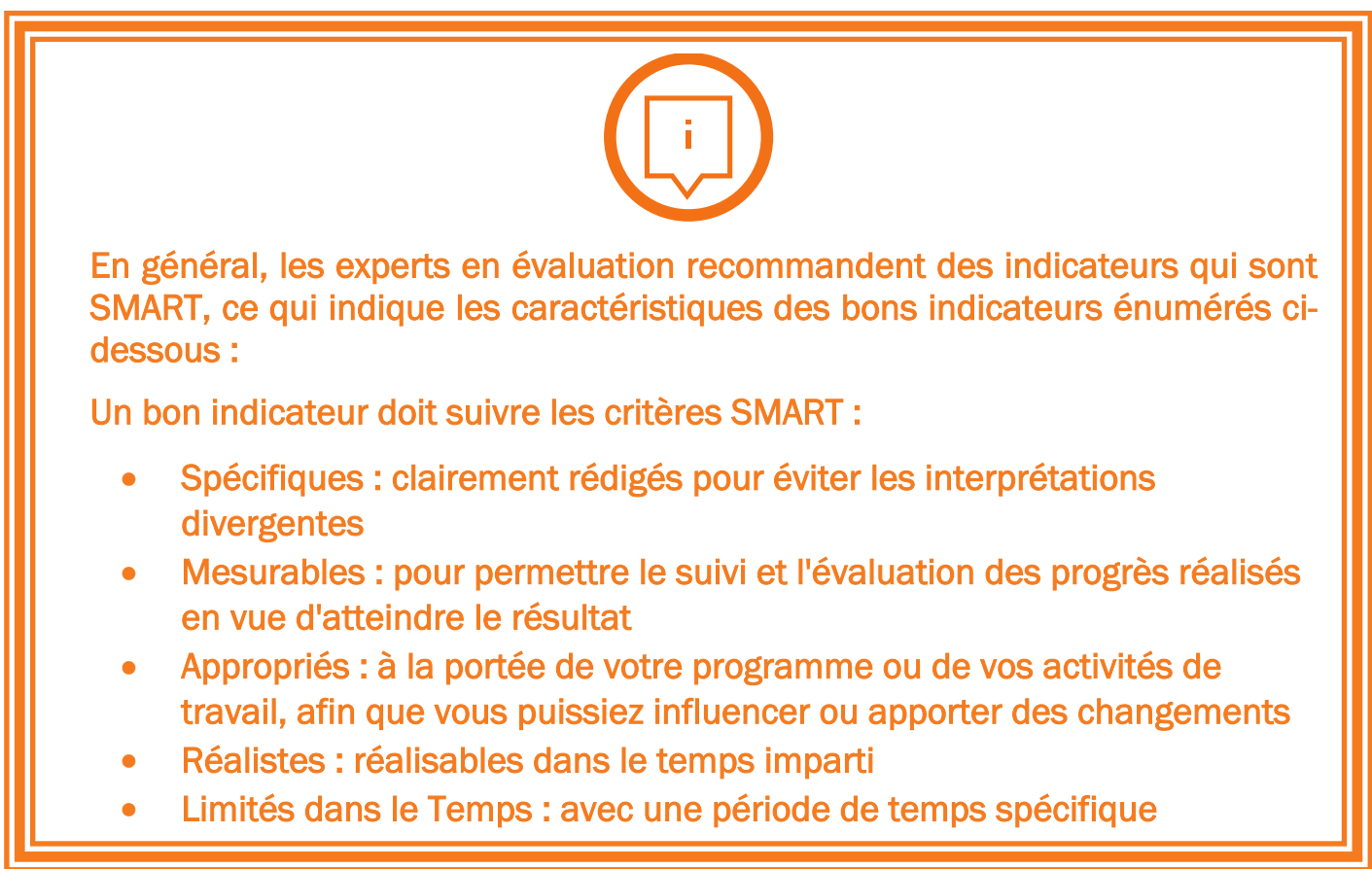

Lorsque vos indicateurs ont été identifiés, se référer au manuel de suivi et d'évaluation des espaces sûrs pour un exemple de : "Tableau de Suivi des indicateurs".

\section{Les bonnes pratiques: Le rôle des mentores dans le suivi}

Le suivi des mentores peut être fait de manière différente en fonction des objectifs définis à atteindre. L'utilisation de contrôles réguliers et de certains outils de suivi peut servir à déterminer si les AJF assistent aux réunions, si l'" espace sûr " est perçu comme vraiment sûr et si la mentore suit le programme d'études comme prévu. Les AJF peuvent également fournir un retour d'information sur leurs mentores. Lors des réunions périodiques, les mentores peuvent partager leurs difficultés et leurs succès. Même les programmes faisant l'objet d'un suivi intensif ne peuvent pas fournir un retour d'information constant.

\section{L'importance de la qualité des données statistiques sur les activités des espaces sûrs $\underline{62}$}

Les données statistiques sur les services et activités fournis aux AJF sont collectées à l'aide des formulaires remplis par les mentores, bien que celles-ci soient rarement formées en la matière. Les gestionnaires de programmes devraient fournir une formation initiale et continue aux prestataires de services sur le remplissage des formulaires, qui pourrait être recyclée au besoin.

62 Population Council: "From research, to program design, to implementation programming for rural girls in Ethiopia a toolkit for practitioners" 
Très souvent, les données collectées auprès des mentores sont de mauvaise qualité : certaines informations sont manquantes et le système de codage est mal appliqué par moment. Il est de la responsabilité du superviseur de s'assurer du bon remplissage des formulaires et d'expliquer l'importance de ces données pour le succès global du programme.

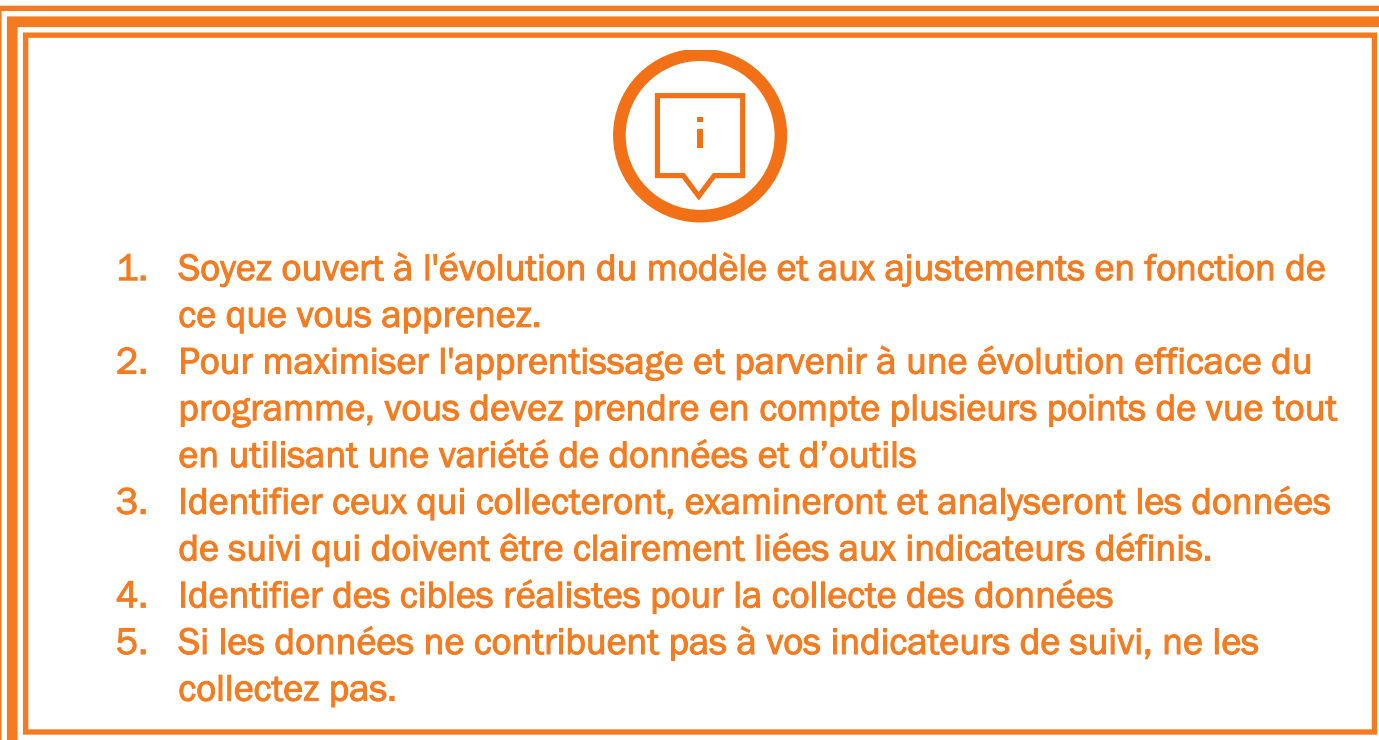

\section{DES NORMES MINIMALES SUR CE QU'IL MESURE POUR ÉVALUER VOTRE SUCCÈS}

\section{Un but et un objectif clairement définis pour l'évaluation des espaces sûrs}

L'évaluation est un processus systématique qui tente de déterminer objectivement la pertinence et l'efficacité des activités par rapport aux objectifs à atteindre. Lorsque vous élaborez vos objectifs d'évaluation, reportez-vous à l'objectif global de votre programme et pensez aux éléments suivants :

- Quels réalisations (effets directs) votre programme essaie-t-il d'atteindre (ex. I'utilisation des services de santé reproductive, l'augmentation d'âge du mariage d'AJF, la réinscription scolaire)?

- Ce qu'il est possible de mesurer compte tenu du calendrier de votre projet ainsi que des ressources et informations disponibles.

- Ce qui se passe en outre dans les communautés concernées par mon projet et qui pourrait également influencer les objectifs/résultats de mon programme.

Les plans d'évaluation ne doivent pas être laissés pour la fin de votre programme mais devrait être un élément central de la planification et la conception générale. A la phase initiale du cycle de planification, vous devez décider de quel type d'évaluation vous voulez faire et définir la matière à évaluer.

Les évaluations peuvent être menées pendant la période du projet ou à la fin de celle-ci, selon l'objectif de l'évaluation. Contrairement au suivi, l'évaluation implique la collecte de données ponctuelles. Cela peut inclure des enquêtes de base à mi-parcours et finales. L'évaluation nécessite une comparaison des changements mesurables du niveau/de la prévalence des variables de résultats avant et après les interventions. 
À ÉVITER : Dépassez votre capacité d'évaluation, ou recueillir plus d'informations qu'il n'en faut.

\section{Déterminez les aspects de votre programme à évaluer pour mesurer son plein succès}

Il est souvent difficile pour les chargés(es) de programme de savoir ce qu'il faut évaluer. Encore une fois, référez -vous à vos objectifs. Qu'est-ce que votre projet vise à accomplir? Quelles données devez-vous collecter pour déterminer si vous avez atteint ces objectifs ? Quelles données sont à collecter en plus des données de suivi ?

Bien que les évaluations puissent sembler difficiles, elles ne le sont pas nécessairement. Concentrez-vous sur les objectifs clés de votre programme.

Voici quelques considérations clés que votre programme devrait envisager à évaluer :

1. Mesurer l'ensemble des compétences de protection que vous voulez que les AJF développent tout au long de leur apprentissage est une excellente façon d'évaluer le succès de votre programme. Votre sélection de mesures spécifiques devrait tenir compte de la variété des compétences de protection dont les AJF ont besoin en fonction de leur âge, de leur l'état civil, des risques associés aux changements de saison, et d'autres différences. À noter également que certaines compétences de protection peuvent être développées et mesurées dans un court laps de temps (six mois), tandis qu'il peut falloir un an ou plus pour en développer d'autres.

a. Exemple: mon programme a-t-il augmenté la confiance en soi des AJF participantes ? Referez-vous au "Questionnaire pour l'évaluation de la confiance en soi " dans le manuel SWEDD de S\&E pour évaluer avec la participation des filles les changements dans leur vie.

b. Exemple : les filles perçoivent-elles un changement dans leur vie depuis qu'elles ont rejoint le programme ? Referez-vous au " Feux tricolore pour l'évaluation des changements dans la vie des filles " dans le manuel SWEDD de S\&E pour évaluer avec la participation des filles les changements dans leur vie

c. Exemple : quelles sont les tendances en matière d'utilisation des services de santé par les filles, du début à la fin de mon programme?

2. Avant d'évaluer si votre programme a influencé les résultats escomptés, il faut envisager d'évoquer les étapes intermédiaires qui contribuent à ce résultat. Par exemple,

a. Comment la connaissance des thèmes du planning familiale et santé reproductive a-t-elle évolué chez mes participantes?

b. Quels sont les compétences de protection que votre programme donne aux AJF pour les aider à atteindre cet objectif?

3. II est également utile de mesurer les changements opérés au niveau de la communauté qui héberge le programme : les AJF sont-elles perçues différemment ? Est-ce qu'elles sont plus mobiles qu'elles ne l'étaient avant dans la communauté ? 
Une fois que vous avez identifié une partie spécifique de votre programme ou un résultat que vous souhaitez évaluer, vous devez ensuite définir :

- Les indicateurs SMART que vous utiliserez pour représenter vos résultats ; et

- Les données dont vous avez besoin pour mesurer ces résultats.

Vos mesures ou standards d'évaluation, appelés " indicateurs ", devraient se concentrer au niveau des AJF elles-mêmes pour évaluer l'effet du programme. Une liste d'exemples d'indicateurs des compétences de protection peut vous aider lorsque vous envisagez la manière d'évaluer l'effet de votre programme ${ }^{63}$. N'oubliez pas qu'il est essentiel que les indicateurs soient Spécifiques, Mesurables, Approprié, Réaliste, et Limité dans le Temps (voir la boîte à la page 75).

Soyez sûr de collecter les données nécessaires pour établir des rapports en fonction de vos indicateurs. Ces données vous permettront de ressortir le niveau de mise en œuvre des activités, les principaux résultats atteints, le niveau d'atteinte des indicateurs, les gaps, etc.

\section{DES NORMES MINIMALES POUR L'ÉTABLISSEMENT D'UN SYSTÈME D'APPRENTISSAGE}

\section{Un but et un objectif clairement définis pour l'apprentissage des espaces sûrs}

Les données de suivi et d'évaluation relatives aux programmes ou aux services sont souvent sousutilisées. Bien que très souvent utilisées pour rédiger les rapports à soumettre aux donateurs du projet ou encore aux autorités supérieures, leur plus grande valeur est souvent négligée. Ce défi peut être surmonté en établissant une culture d'apprentissage qui permettra aux responsables de la mise en œuvre et aux gestionnaires du projet de faire une évaluation approfondie de la conception et de la mise en œuvre du programme tout en apportant des corrections stratégiques.

$\mathrm{Au}$ vu et à l'examen de certains indicateurs, le personnel du programme peut donner des informations précieuses sur l'avancée et les performances du programme. En collectant des données et en tirant des enseignements tout au long de la mise en œuvre, vous pouvez éviter le piège de découvrir qu'il y avait un obstacle lorsque vous effectuez votre évaluation finale et prendre des mesures pour surmonter ce défi avant la fin du projet.

La conception et le suivi des programmes destinés aux AJF doivent être appuyés d'une culture d'utilisation et d'apprentissage des données. Le meilleur moyen de favoriser une culture d'utilisation des données est de faire en sorte que les responsables et le personnel de mise en œuvre voient la valeur inhérente des données et leur avantage pour le programme et pour les AJF

\footnotetext{
63 Population Council " Développer les compétences de protection des AJF: Ensemble d'Outils pour la Conception d'un Programme "
} 
que nous soutenons. ${ }^{64}$ Les méthodes de recherche et l'utilisation des données doivent être démystifiées et rendues pratiques et accessibles aux gestionnaires et au personnel des programmes. Les preuves générées doivent être exploitables et non pas abstraites, et doivent fournir des informations suffisamment détaillées pour donner une orientation pratique et de bon sens aux programmes destinés aux AJF.

Le retour d'information (feedback) est essentiel à l'apprentissage, au renforcement des capacités et à l'amélioration des performances (Figure 10).

Figure 10 : La boucle de feedback du SEA

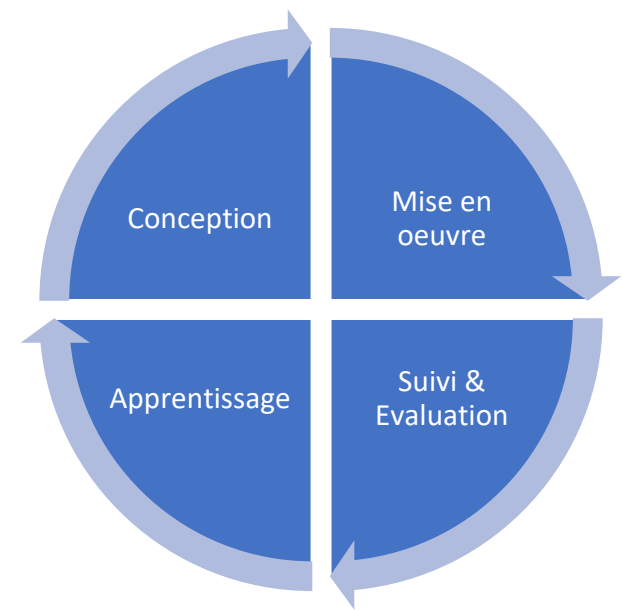

$\underline{\text { Une boucle de feedback qui peut améliorer et informer la conception et la mise en œuvre }}$

Un mécanisme de retour d'information doit être intégré dans la structure de gestion du programme pour garantir que les gestionnaires ne manquent pas une occasion d'apprendre. Le feedback doit être basé sur des données de suivi et doit être fourni aux parties prenantes à tous les niveaux de la structure organisationnelle.

Les systèmes de suivi sur support papier peuvent entraîner une lourde charge de travail pour la saisie des données, ce qui peut provoquer un important arriéré de données. Plus particulièrement dans les grands programmes, la rationalisation des processus SEA par l'abandon des systèmes sur papier au profit de la numérisation permet de suivre en temps utile les indicateurs de base. Les données numériques de S\&E peuvent alimenter une base de données de suivi, qui amène le personnel formé à créer des tableaux de bord permettant aux gestionnaires et aux superviseurs de renforcer l'efficacité de leur mentorat et de résoudre les problèmes avant qu'ils ne s'enracinent. II peut également permettre des contrôles réguliers d'assurance qualité à tous les niveaux. ${ }^{65}$

64 Population Council: « From research, to program design, to implementation programming for rural girls in Ethiopia a toolkit for practitioners" https://www.popcouncil.org/uploads/pdfs/2011PGY EthiopiaGirlsProgramToolkit.pdf

65Temin, Miriam avec: Arune Estavela, Craig Heck, Natalie Jackson, Joana Mendes Population Council:

"Positioning Community-Based Girl Group Programs For Success Lessons Learned From Population Council's Technical Assistance Partnership With UNFPA: Mozambique's Rapariga Biz"

https://knowledgecommons.popcouncil.org/departments_sbsr-pgy/1205/ 
Certains pays SWEDD sont en train de piloter un system de collecte des données de suivi numérique. C'est une stratégie qui a le potentiel de rendre les données de contrôle de la qualité facilement accessibles, ce qui peut améliorer l'efficacité du retour d'informations à tous les niveaux de la mise en œuvre des programmes.

\section{Considérations pour fournir un retour d'information sur les performances individuelles et les performances globales du programme aux mentores}

L'un des rôles clés des responsables de programme est de fournir un retour d'information constructives aux mentores. La disponibilité d'informations en retour doit être considérée comme un indicateur d'un système de suivi fonctionnel. Les réunions des mentores et les visites de supervision sont l'occasion de fournir un retour d'information aux mentores et de réorienter les programmes afin de combler les lacunes.

Les éléments clés du retour d'information sont les suivants :

- II devrait être basé sur un suivi ou une évaluation solide et structuré(e), par exemple, sur la base d'une analyse des données obtenues à partir des listes de contrôle.

- Il peut être verbal ou écrit avec des points d'action clés.

- II doit être donné avec une attitude positive pour motiver et réduire les obstacles futurs. Une attitude négative peut mettre le travailleur sur la défensive et nuire à la relation de travail.

- II doit inclure un dialogue commençant avec le travailleur qui partage ses propres forces, faiblesses et défis

- II doit être régulier et opportun pour améliorer les performances.

- Le retour d'information au travailleur responsable de l'activité est plus facile s'il tient à jour un rapport d'activité standardisé. 66

Dans la boîte à outils du Population Council, "Valoriser le potentiel des mentores" il existe plusieurs outils (ex. check-lists) qui mettent en évidence les types d'informations importantes à transmettre aux mentores afin d'identifier et de traiter les problèmes et d'améliorer les performances individuelles. Ces types d'information incluent :

- Date, nom de la mentore, numéro de groupe.

- Données de la réunion soumises?

- Session pertinente facilitée?

- Curriculum suivi ?

- Faible taux de fréquentation observé ?

- Nombre élevé d'AJF hors programme?

- Besoin d'un suivi?

- Mesures prises après le suivi.

66 Population Council: "Training manual on basic monitoring and evaluation of social and behavior change communication health programs" https://www.popcouncil.org/uploads/pdfs/2014RH_BCCTrainingManual.pdf 
Outre la présence et le respect du programme d'études, les outils de contrôle doivent permettre au superviseur d'évaluer le niveau de compétence dont la mentore fait preuve lors de l'animation de la session de l'espace sûr en documentant sa capacité à :

- Être patiente,

- S'amuser avec les filles et à être enthousiaste,

- Utiliser des exemples pratiques pertinents pour la vie des AJF,

- Être empathique, attentionnée et sensible,

- Être préparée pour la session et bien gérer le temps,

- Résoudre les conflits,

- Montrer la compréhension du matériel,

- Simplifier les concepts/le langage pour la compréhension des AJF, et

- Promouvoir le leadership en permettant aux AJF de participer, de guider des activités et d'assumer des rôles de direction.

\section{Considérations relatives à la fourniture d'un retour d'information aux autres parties prenantes du programme}

Pour optimiser la valeur du SEA, faites-en sorte que le SEA soit l'affaire de tous. La compréhension des outils et des processus de SEA doit s'étendre au-delà des responsables ou des équipes de suivi et d'évaluation désignés, du personnel chargé de la collecte des informations, comme les superviseurs des mentores et les autres partenaires de mise en œuvre. Cela sera possible lorsque les membres du personnel reconnaîtront la valeur et l'applicabilité des données SEA, et comprendront l'importance de la bonne collecte et de la bonne transmission des données ${ }^{67}$.

Au niveau national et régional, les données de suivi peuvent être utilisées (par les UGP SWEDD, les ministères, les points focaux régionaux etc.) pour la coordination, le plaidoyer et pour informer la prise de décision concernant les corrections de trajectoire, les plans d'évaluation, la planification pour l'avenir.

Le suivi et l'évaluation doivent aussi viser une redevabilité à la communauté qui a contribué à l'implémentation des espaces sûrs en espérant des améliorations.

67 Temin, Miriam avec: Arune Estavela, Craig Heck, Natalie Jackson, Joana Mendes Population Council:

"Positioning Community-Based Girl Group Programs For Success Lessons Learned From Population Council's Technical Assistance Partnership With Unfpa: Mozambique's Rapariga Biz"

https://knowledgecommons.popcouncil.org/departments_sbsr-pgy/1205/ 


\section{DES NORMES MINIMALES POUR LA COLLECTE ET L'UTILISATION DE DONNÉES SEA DE QUALITÉ}

Avant, pendant et après la mise en œuvre du programme, utilisez la recherche quantitative et qualitative pour générer les données nécessaires à l'identification des meilleures pratiques, pour affiner les éléments critiques du programme et pour éliminer les approches inefficaces. Réfléchissez à qui utilisera les données de suivi et d'évaluation pour l'apprentissage du programme et réfléchissez de manière pratique aux sources de ces données (quantitatif et qualitatif) et pensez à qui sera responsable de la collecte de chaque type de données :

- Listes de présence,

- Rapports de mentorat,

- Enquêtes connaissances, attitudes, pratiques (CAP),

- Observation (visite de site),

- Entretiens approfondis qualitatifs, ou

- Groupes de discussion (avec les bénéficiaires).

Chaque méthode est particulièrement adaptée pour obtenir un type de données ou un avis spécifique sur un aspect de votre programme ${ }^{68}$. Ces données peuvent être utilisées par les différents acteurs de votre programme. Au sujet de l'apprentissage interne aux espaces sûrs, les données de suivi et d'évaluation peuvent être utilisées par les mentores et leurs superviseurs afin de relever les défis observés lors de la mise en œuvre. Ces données sont aussi utiles pour les autres (AJF, membres de la communauté, partenaires de la mise en œuvre, UGP, décideurs, etc.) de manière à améliorer l'environnement et à s'assurer qu'il est favorable à la mise en œuvre et la réussite des espaces sûrs, et pour apprendre de l'échec. Les données de suivi peuvent être collectées tous les trois à six mois.

Si vous cherchez à vous informer sur la communauté, pensez à engager les AJF inscrites à votre programme comme assistantes de recherche. Avec une formation minimale, elles peuvent se rendre dans la communauté et y collecter l'information désirée sur différentes questions et ressources : services de santé, écoles, sécurité et programmes de jeunesse, notamment.

Cette approche présente plus d'un avantage : 1) les AJF de votre programme disposent d'un accès et d'une connaissance unique de leur communauté ; 2) elles auront à l'égard du programme un sentiment d'appartenance plus profond, tiré de l'information qu'elles auront collectée ; et 3) l'approche développe d'importantes qualités de leadership et compétences techniques chez les participantes.

\section{Utiliser des méthodes à la fois qualitatives et quantitatives pour générer des données de suivi et d'évaluation}

La recherche quantitative est la mesure des phénomènes en termes de quantité ou nombre. La recherche qualitative consiste à comprendre le processus qui conduit au résultat. Elle se concentre

\footnotetext{
68 Population Council: "Training manual on basic monitoring and evaluation of social and behavior change
} communication health programs" 
sur les perceptions et les expériences des participantes qui conduisent à la manière dont elles prennent des décisions et agissent 69 .

Tableau 9 : Caractéristiques des données quantitatives et qualitatives

\begin{tabular}{|c|c|c|}
\hline Caractéristiques & Quantitative & Qualitative \\
\hline $\begin{array}{l}\text { Objectif } \\
\text { analytique }\end{array}$ & $\begin{array}{l}\square \text { Quantifier la variation. } \\
\square \text { Décrire les caractéristiques d'une } \\
\text { population. }\end{array}$ & $\begin{array}{l}\square \text { Décrire la variation. } \\
\square \text { Décrire les expériences } \\
\text { individuelles et les normes } \\
\text { de groupe. }\end{array}$ \\
\hline $\begin{array}{l}\text { Format des } \\
\text { questions }\end{array}$ & $\begin{array}{l}\square \text { Questions fermées (choisir les } \\
\text { réponses parmi les options } \\
\text { proposées) ou semi-structurées }\end{array}$ & $\begin{array}{l}\square \text { Questions ouvertes (La } \\
\text { réponse est descriptive) }\end{array}$ \\
\hline $\begin{array}{l}\text { Format des } \\
\text { données }\end{array}$ & $\square$ Les résultats sont donnés en chiffres & $\begin{array}{l}\square \text { Les résultats sont donnés } \\
\text { en mots }\end{array}$ \\
\hline $\begin{array}{l}\text { Conception des } \\
\text { outils }\end{array}$ & $\begin{array}{l}\square \text { La conception de l'outil de collecte de } \\
\text { données est stable du début à la fin. } \\
\square \text { Les réponses des participants } \\
\text { n'influencent ni ne déterminent } \\
\text { comment et quelles questions les } \\
\text { chercheurs poseront ensuite. } \\
\text { L'enquêteur doit poser et suivre le } \\
\text { même ordre de questions que celui } \\
\text { indiqué dans le questionnaire. }\end{array}$ & $\begin{array}{l}\square \text { II existe une certaine } \\
\text { souplesse dans la manière } \\
\text { dont les questions sont } \\
\text { posées. } \\
\square \text { La réponse des participants } \\
\text { influe sur la manière dont } \\
\text { les chercheurs vont ensuite } \\
\text { poser les questions et sur } \\
\text { les questions qu'ils vont } \\
\text { poser. L'ordre des questions } \\
\text { n'est pas rigide. }\end{array}$ \\
\hline
\end{tabular}

\section{Caractéristiques de la recherche qualitative ${ }^{70}$ :}

- Elle explore les réponses au quoi, au pourquoi et au comment des décisions et des actions prises

- Elle explore et découvre les phénomènes sociaux dans le processus de décision à l'action

- Elle fournit un aperçu de la signification des décisions et des actions

- Elle laisse la répondante décrire ses réponses

- Elle est interactive plutôt que fixe

- Elle implique les répondantes en tant que participantes actives plutôt que sujets

69 Population Council: "Training manual on basic monitoring and evaluation of social and behavior change communication health programs"

70 Adapté de: "Training manual on basic monitoring and evaluation of social and behavior change communication health programs" du Population Council 
Vous avez besoin d'une recherche qualitative dans votre S\&E parce qu'elle vous fournira des données qualitatives de l'AJF, de ses mentores et de la perspective de leur communauté sur le programme. Ainsi, la recherche qualitative vous permet :

- D'améliorer la qualité du programme : Les méthodes de suivi qualitatif permettent de recueillir des données sur la qualité de la mise en œuvre. Elles sont nécessaires à l'apprentissage, à la replanification et à la prise en compte de la qualité d'un programme.

- De comprendre le point de vue des participantes : Les méthodes qualitatives permettent de comprendre pourquoi les participantes font ce qu'elles font. Elles fournissent des informations sur l'aspect humain d'une question - à savoir les comportements, croyances, opinions, émotions et relations souvent contradictoires des individus.

- D'expliquer les résultats quantitatifs : Les méthodes qualitatives sont plus exploratoires, elles complètent les méthodes quantitatives en explorant les raisons, les schémas qui sous-tendent les réponses des méthodes d'enquête quantitatives.

- Lorsqu'elles sont utilisées en complément des méthodes quantitatives, les méthodes qualitatives peuvent nous aider à interpréter et à mieux comprendre la réalité complexe d'une situation donnée et les implications des données quantitatives.

\section{ÉTUDE DE CAS \\ L'UTILISATION DES MÉTHODES QUALITATIVES POUR LE SUIVI D'UN PROGRAMME BIRUH TESFA}

Biruh Tesfa est un programme basé sur des données probantes qui fournit aux AJF marginalisées non scolarisées des zones urbaines d'Éthiopie l'éducation, les ressources et les compétences dont elles ont besoin pour améliorer leurs capacités de lecture, d'écriture et de calcul, ainsi que leur santé et leur bien-être.

Tous les six mois, par exemple, les responsables du programme Biruh Tesfa ont interrogé entre 10 et $20 \mathrm{AJF}$, ainsi que 5 à 10 mentores. Les responsables ont utilisé ces entretiens pour explorer de nouveaux domaines d'expansion ou de modification du programme, et ils modifient les questions à chaque tour pour obtenir différents types d'informations. C'est un exemple qui montre comment le suivi qualitatif après la mise en œuvre des programmes peut fournir des informations importantes pour l'amélioration des programmes.

Adapté de " Population Council: " Building the Assets to Thrive Addressing the HIV-related vulnerabilities of adolescent girls in Ethiopia "

https://www.popcouncil.org/uploads/pdfs/2014PGY BuildingAssetsThrive.pdf"

\section{$\underline{\text { S'assurer que les données sont crédibles, valides, fiables, et utilisables }}$}

Au cours de votre processus SEA, il est très important de vous assurer que les données que vous collectez sont de bonne qualité. Ceci est important parce que les données de mauvaise qualité sont trompeuses mais aussi parce qu'elles auront un impact sur votre programme si vous décidez de faire des ajustements en vous basant sur ces données-là. Voir ci-après quelques moyens qui vous aideront à vous assurer que vos données sont de bonne qualité. 
Veiller à ce que les outils de suivi et d'évaluation recueillent avec précision les informations appropriées

Les indicateurs sont des mesures spécifiques qui sont définis par les objectifs du programme. Ils sont utilisés dans le suivi et l'évaluation des performances du programme et doivent aider à évaluer dans quelle mesure l'intervention a changé les résultats.

En outre, les nouveaux outils de collecte de données et les outils existants validés doivent être testés au préalable dans votre contexte afin de s'assurer que les questions sont comprises par votre groupe cible et que les outils recueillent de manière fiable les informations prévues.

La qualité des données peut également être assurée lors du nettoyage des données

Des directives/ procédures standards sur la gestion des données doivent être élaborées avant la collecte et être suivies tout le long et pendant les contrôles d'assurance qualité. Une personne qui n'a pas participé directement à la collecte des données doit effectuer, si cela est possible, des contrôles de qualité ponctuels par rapport aux sources de données primaires afin d'identifier et de résoudre toute divergence peu après ou pendant la collecte des données.

\section{Ressources supplémentaires de SEA}

Dans ce chapitre, nous avons passé en revue quelques exemples d'outils et de types de données à collecter. Nous vous encourageons à consulter d'autres ressources pour vous aider à éclairer vos outils de collecte de données et à formuler des questions spécifiques dans le cadre d'un plan SEA rigoureux et facile à mettre en œuvre. Le tableau ci-dessous fournit quelques ressources supplémentaires. Les équipes de SWEDD SEA dans d'autres pays peuvent également avoir des exemples forts à partager.

Tableau 10 : Ressources supplémentaires de SEA

\begin{tabular}{|c|c|c|}
\hline Titre & Langue & Disponible \\
\hline $\begin{array}{l}\text { AGI-K Adolescent Girls Initiative Baseline } \\
\text { Survey Instruments }\end{array}$ & English & $\begin{array}{l}\text { https://www.popcouncil.org/ } \\
\text { uploads/pdfs/2015PGY_AGl- } \\
\text { K_BaselineSurveylnstruments } \\
\text {.pdf }\end{array}$ \\
\hline $\begin{array}{l}\text { AGI-K Adolescent Girls Initiative Midline } \\
\text { Survey Instruments }\end{array}$ & English & $\begin{array}{l}\text { https://www.popcouncil.org/upl } \\
\text { oads/pdfs/2017PGY AGI- } \\
\text { K MidlineSurveylnstruments.xlsx }\end{array}$ \\
\hline
\end{tabular}




\section{CONCLUSION}

Un plan SEA judicieux, ciblé et raisonnable peut contribuer à garantir le respect des délais du programme, l'atteinte de ses objectifs ainsi que ses progrès. Assurez-vous que votre programme dispose d'un plan SEA au début du programme et que vous vous y référez régulièrement.

Reportez-vous à ce guide pour vous assurer que votre plan SEA comporte les normes minimales nécessaires, et en particulier que vous :

1. Donnez la priorité à des questions spécifiques de suivi, d'évaluation et d'apprentissage. Qu'ils sont pertinents par rapport à l'objectif global de votre projet.

2. Identifiez le type de données que vous devrez collecter et les outils appropriés qui vous aideront à collecter ces données lorsque vous auriez déterminé vos questions SEA.

a) Rappelez-vous ! Ne collectez que les données qui sont crédibles, exploitables, responsables, transportables.

b) Veillez à ce que vos données se rapportent aux indicateurs que vous avez établis et qui sont : Spécifiques, Mesurables, Appropriés, Réalistes, et Limités dans le Temps.

3. Exploitez les visites sur site pour collecter des données de surveillance et/ou d'évaluation.

4. Utilisez les mentores pour collecter les données de suivi.

5. Interviewez les AJF et les mentores pour comprendre comment le programme se déroule, ce qui va bien, ce qui peut être amélioré, et comment le programme influence leurs compétences et leurs vies. 
APPENDICE 
Développer les compétences de protection des filles: Ensemble d'Outils pour la Conception d'un Programme [English et Français ]

Un ensemble d'outils et de feuilles de travail pratiques et conviviaux, la collection Développer les compétences de protection des filles aide ceux qui conçoivent et mettent en œuvre des programmes pour les filles à intégrer une approche de renforcement des compétences de protection pour améliorer leur couverture. Les exercices et les outils présentés mettent l'accent sur les programmes qui se sont avérés efficaces pour réduire les risques encourus par les filles et élargir leurs possibilités. Cette collection aidera le personnel des programmes à concevoir, réévaluer, conduire, suivre et évaluer leurs activités, à n'importe quel stade de la planification ou de la mise en œuvre.

La Boîte à Outils: renforcement des compétences: développer des repères positifs pour les adolescentes (The Building Assets Toolkit) [English et Français]

La communauté de pratique des Adolescentes et ses partenaires ont été les premiers à utiliser le Building Assets Toolkit@ pour aider au développement d'un contenu de programme pertinent, adapté et positif. Cette boîte à outils guide les praticiens à travers une approche de développement des compétences pour les aider à élaborer des points de référence progressifs et réalisables pour les filles par âge, catégorie sociale et contexte. II modélise la pensée critique nécessaire à la conception de programmes efficaces.

\section{Plus qu'une toile de fond (More than a backdrop) [English]}

Ce guide se concentre sur cinq questions courantes posées par les praticiens sur leur lieu de travail. À l'aide d'exemples concrets, le guide "More than A Backdrop Action Guide" montre comment l'accès à des informations sur la communauté peut faciliter la mise en œuvre et augmenter les chances de succès en aidant le personnel du programme à s'adapter et à prendre des décisions éclairées dès la conception du programme. Cela peut influencer les décisions relatives au programme, telles que la sélection des personnes éligibles, la création du contenu du programme et l'évaluation des besoins de la communauté.

Valoriser le potentiel des mentors: Recrutement, formation et appui des mentors pour des programmes dédiés aux adolescents (Making the Most of Mentors : Recruitment, Training, and Support of Mentors for Adolescent Girl Programming) [English et Français]

Cette boîte à outils rassemble des leçons, des conseils et des idées qui peuvent être adaptés et utilisés pour trouver, former, suivre, soutenir et évaluer les mentors. Les informations contenues dans la boîte à outils "Valoriser le potentiel des mentors" sont basées sur des documents provenant de programmes pour adolescentes mis en œuvre par le Population Council. Les ressources sont destinées aux planificateurs de programmes, aux superviseurs de mentors et aux mentors, et présentent des études de cas réels comme exemples d'application pratique de ces activités et outils. Toutes les ressources peuvent être adaptées aux contextes locaux. 


\section{CONTRIBUTIONS}

\section{RÉDACTRICES}

Karen Kirk, Population Council

Aissata Ba, Population Council

Sara Chace Dwyer, Population Council

Miriam Temin, Population Council

\section{CONTRIBUTEURS ET REVUE}

SWEDD Benin

SWEDD Burkina Faso

SWEDD Cameroun

SWEDD Côte d'Ivoire

SWEDD Guinée

SWEDD Mali

SWEDD Mauritanie

SWEDD Niger

SWEDD Tchad

UNFPA Benin

UNFPA Burkina Faso

UNFPA Cameroun

UNFPA Côte d'Ivoire

UNFPA Guinée

UNFPA Mali

UNFPA Mauritanie

UNFPA Niger

UNFPA Tchad

UNFPA BRAOC et STR

\section{IMAGES DE COUVERTURE}

En haut à gauche et en bas: Ollivier Gerard, UNFPA

En haut à droite: UNFPA 
